\title{
Silver-Catalyzed Remote C5-H Selenylation of Indoles
}

\author{
Wei-Hong Song, ${ }^{\dagger}{ }^{J i a}$ Shi,${ }^{\dagger}$ Xiaohong Chen ${ }^{*}, \dot{a}$ and Guoyong Song $*, \dagger$
}

$†$ †eijing Advanced Innovation Center for Tree Breeding by Molecular Design, Beijing Key

Laboratory of Lignocellulosic Chemistry, Beijing Forestry University, No.35 Tsinghua East Road,

Beijing, 100083, P.R. China

†Center for Lignocellulose Chemistry and Biomaterials, Dalian Polytechnic University, No.1

Qinggongyuan, Dalian, Liaoning, 116034, P.R. China

\author{
*G.S email: songg@bjfu.edu.cn \\ *X.C email: xhchen@ dlpu.edu.cn
}

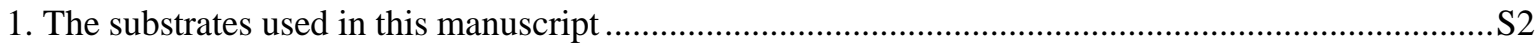

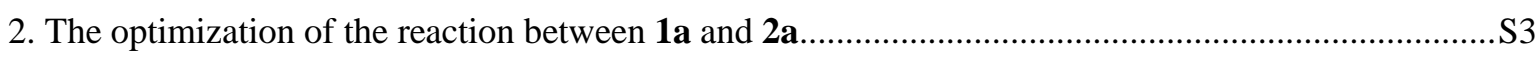

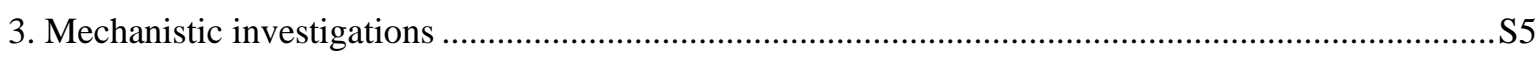

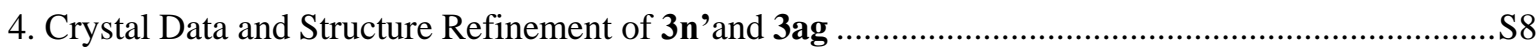

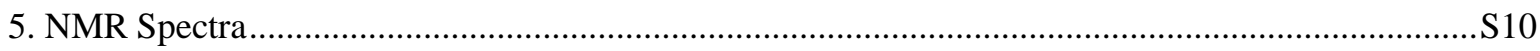




\section{The substrates used in this manuscript}
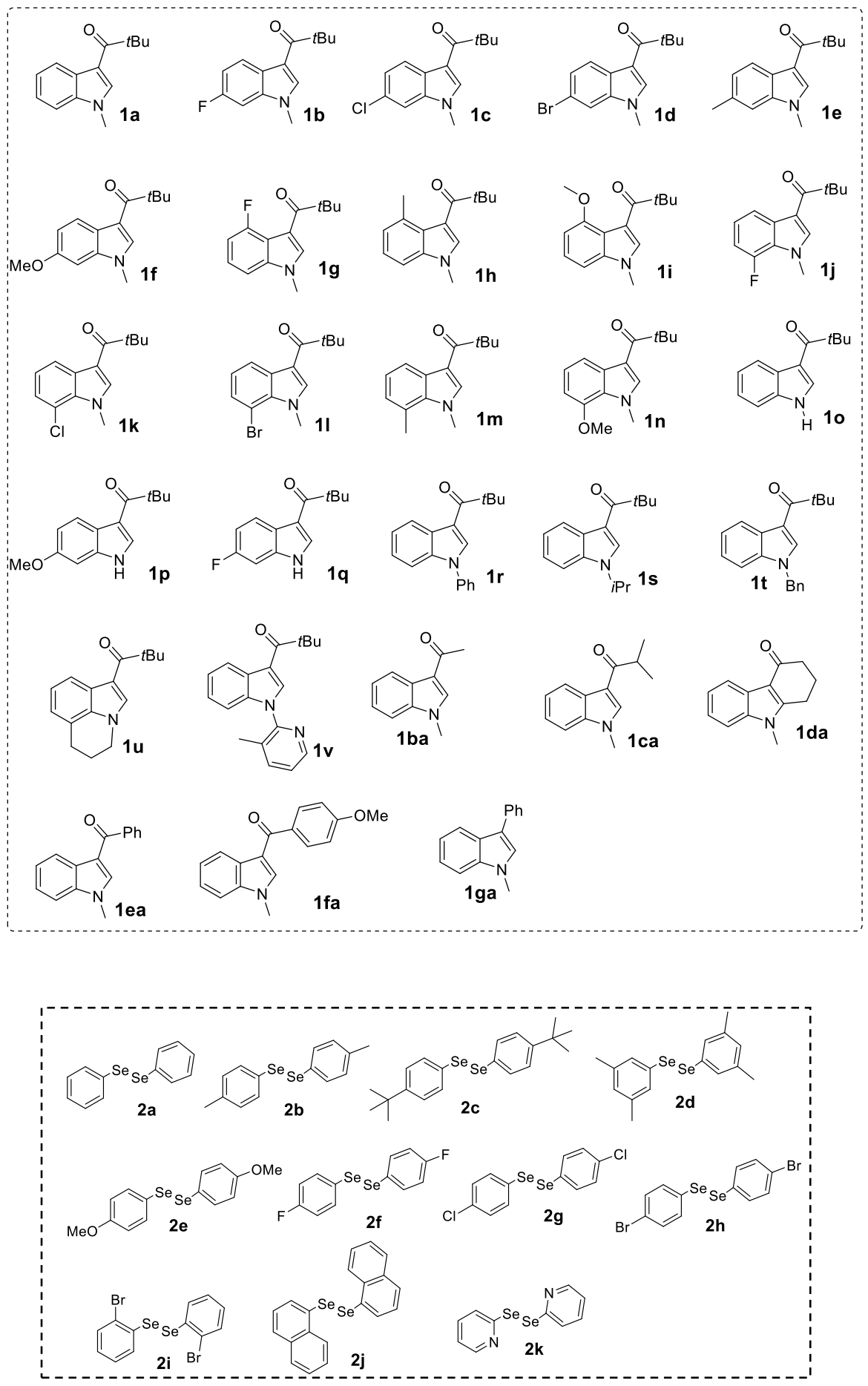
2. The optimization of the reaction between 1a and $2 a$

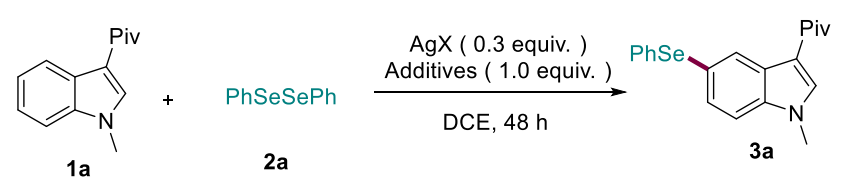

$1 \mathrm{a}$

\begin{tabular}{|c|c|c|c|c|c|c|}
\hline Entry & Solvent & $\operatorname{AgX}$ & Additives & $\mathrm{T} /\left({ }^{\circ} \mathrm{C}\right)$ & Con. $(\%)^{b}$ & Yield $(\%)^{c}$ \\
\hline 1 & DCE & $\mathrm{AgSbF}_{6}$ & / & R.T. & 30 & 30 \\
\hline 2 & DCM & $\mathrm{AgSbF}_{6}$ & / & R.T. & 29 & 29 \\
\hline 3 & $\mathrm{THF}$ & $\mathrm{AgSbF}_{6}$ & / & R.T. & 0 & 0 \\
\hline 4 & EA & $\mathrm{AgSbF}_{6}$ & / & R.T. & 0 & 0 \\
\hline 5 & DMSO & $\mathrm{AgSbF}_{6}$ & / & R.T. & 0 & 0 \\
\hline 6 & $\mathrm{MeCN}$ & $\mathrm{AgSbF}_{6}$ & / & R.T. & 0 & 0 \\
\hline 7 & Toluene & $\mathrm{AgSbF}_{6}$ & / & R.T. & 0 & 0 \\
\hline 8 & $\mathrm{CH}_{3} \mathrm{OH}$ & $\mathrm{AgSbF}_{6}$ & / & R.T. & 0 & 0 \\
\hline 9 & DCE & $\mathrm{AgOAc}$ & I & R.T. & 0 & 0 \\
\hline 10 & DCE & $\mathrm{Ag}_{2} \mathrm{O}$ & / & R.T. & 0 & 0 \\
\hline 11 & DCE & $\mathrm{Ag}_{2} \mathrm{CO}_{3}$ & / & R.T. & 0 & 0 \\
\hline 12 & DCE & $\mathrm{AgNO}_{3}$ & / & R.T. & 0 & 0 \\
\hline 13 & DCE & $\mathrm{CF}_{3} \mathrm{SO}_{3} \mathrm{Ag}$ & / & R.T. & 0 & 0 \\
\hline 14 & DCE & $4-\mathrm{CH}_{3} \mathrm{C}_{6} \mathrm{H}_{4} \mathrm{SO}_{3} \mathrm{Ag}$ & / & R.T. & 0 & 0 \\
\hline 15 & DCE & $\mathrm{AgClO}_{4}$ & / & R.T. & 35 & 29 \\
\hline 16 & DCE & $\mathrm{AgBF}_{6}$ & / & R.T. & 28 & 28 \\
\hline 17 & DCE & $\left(\mathrm{C}_{6} \mathrm{~F}_{5}\right)_{3} \mathrm{~B}$ & / & R.T. & 0 & 0 \\
\hline 18 & DCE & $\mathrm{NaSbF}_{6}$ & / & R.T. & 0 & 0 \\
\hline 19 & DCE & $\mathrm{AgSbF}_{6}$ & $\mathrm{CsOAc}$ & R.T. & 29 & 29 \\
\hline 20 & DCE & $\mathrm{AgSbF}_{6}$ & $\mathrm{NaHCO}_{3}$ & R.T. & 30 & 30 \\
\hline 21 & DCE & $\mathrm{AgSbF}_{6}$ & $\mathrm{NaOH}$ & R.T. & 28 & 28 \\
\hline 22 & DCE & $\mathrm{AgSbF}_{6}$ & $\mathrm{NaO}^{t} \mathrm{Bu}$ & R.T. & 0 & 0 \\
\hline 23 & DCE & $\mathrm{AgSbF}_{6}$ & PIDA & R.T. & 55 & 45 \\
\hline 24 & DCE & I & PIDA & R.T. & 0 & 0 \\
\hline 25 & DCE & $\mathrm{AgSbF}_{6}$ & PIFA & R.T. & 55 & 42 \\
\hline 26 & DCE & $\mathrm{AgSbF}_{6}$ & $\mathrm{Cu}(\mathrm{OAc})_{2}$ & R.T. & 30 & 30 \\
\hline 27 & DCE & $\mathrm{AgSbF}_{6}$ & TBAI & R.T. & 0 & 0 \\
\hline 28 & DCE & $\mathrm{AgSbF}_{6}$ & PIDA & 50 & 90 & 66 \\
\hline 29 & DCE & $\mathrm{AgSbF}_{6}$ & PIDA & 70 & 97 & 71 \\
\hline $30^{d}$ & DCE & $\mathrm{AgSbF}_{6}$ & PIDA & 80 & 100 & 82 \\
\hline 31 & DCE & $\mathrm{AgSbF}_{6}$ & PIFA & 80 & 100 & 81 \\
\hline 32 & DCE & $\mathrm{AgSbF}_{6}$ & PIOPiv & 80 & 95 & 79 \\
\hline 33 & DCE & $\mathrm{AgSbF}_{6}$ & DTBP & 80 & 50 & 0 \\
\hline
\end{tabular}




\begin{tabular}{|c|c|c|c|c|c|c|}
\hline & & $\mathrm{AgSbF}_{6}$ & & & & \\
\hline \multirow[t]{2}{*}{34} & DCE & ( 0.25 equiv) & PIDA & 80 & 91 & 55 \\
\hline & & $\mathrm{AgSbF}_{6}$ & & & & \\
\hline \multirow[t]{2}{*}{35} & DCE & ( 0.2 equiv) & PIDA & 80 & 80 & 50 \\
\hline & & $\mathrm{AgSbF}_{6}$ & & & & \\
\hline 36 & DCE & ( 0.1 equiv) & PIDA & 80 & 55 & 40 \\
\hline
\end{tabular}

${ }^{a}$ Unless noted otherwise, the reaction was carried out in $0.1 \mathrm{mmol}$ scale in $1.5 \mathrm{~mL}$ solvent, and the ratio of 1a: $2 \mathrm{a}$ was 1: $0.55 .{ }^{b} \%$ conversions of $1 \mathbf{a}$ was determined after column chromatography. ${ }^{c}$ Yield of isolated product. ${ }^{d}$ The reactions were carried out for $36 \mathrm{~h}$.

PIDA: (diacetoxyiodo)benzene, PIFA: [bis(trifluoroacetoxy)iodo]benzene), PIOPiv: bis(tertbutylcarbonyloxy)iodobenzene, DTBP: (di-tert-butyl peroxide). 


\section{Mechanistic investigations}

General procedure for competition experiments: 1a (43.1 mg, $0.2 \mathrm{mmol}), \mathbf{1 f}(49.1 \mathrm{mg}, 0.2 \mathrm{mmol}), 2 \mathbf{a}(34.3 \mathrm{mg}, 0.55$ equiv.), $\mathrm{AgSbF}_{6}$ (20.6 mg, $30 \mathrm{~mol} \%$ ), PIDA (64.4 mg, $\left.0.2 \mathrm{mmol}\right)$, and DCE (2 mL) were charged into a Schlenk tube under $\mathrm{N}_{2}$ atmosphere. The result mixture was stirred at $50{ }^{\circ} \mathrm{C}$ for $24 \mathrm{~h}$. After that, the mixture was filtered through a short silica gel and then the solvent was removed under reduced pressure to afford a crude product. ${ }^{1} \mathrm{H}$ NMR analysis of crude product indicated that only $\mathbf{3 f}$ was formed, with no observation of $\mathbf{3 c}$.
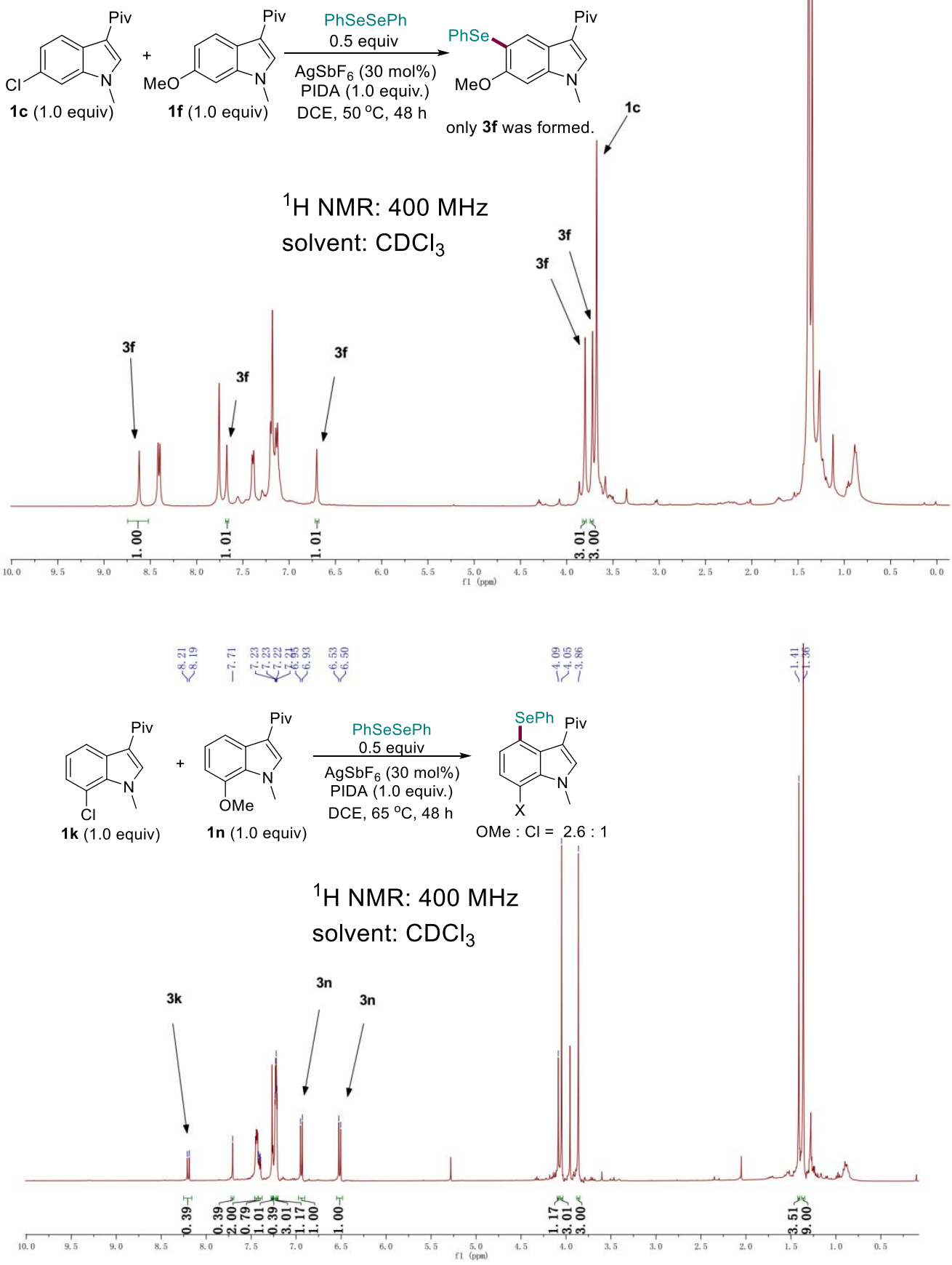


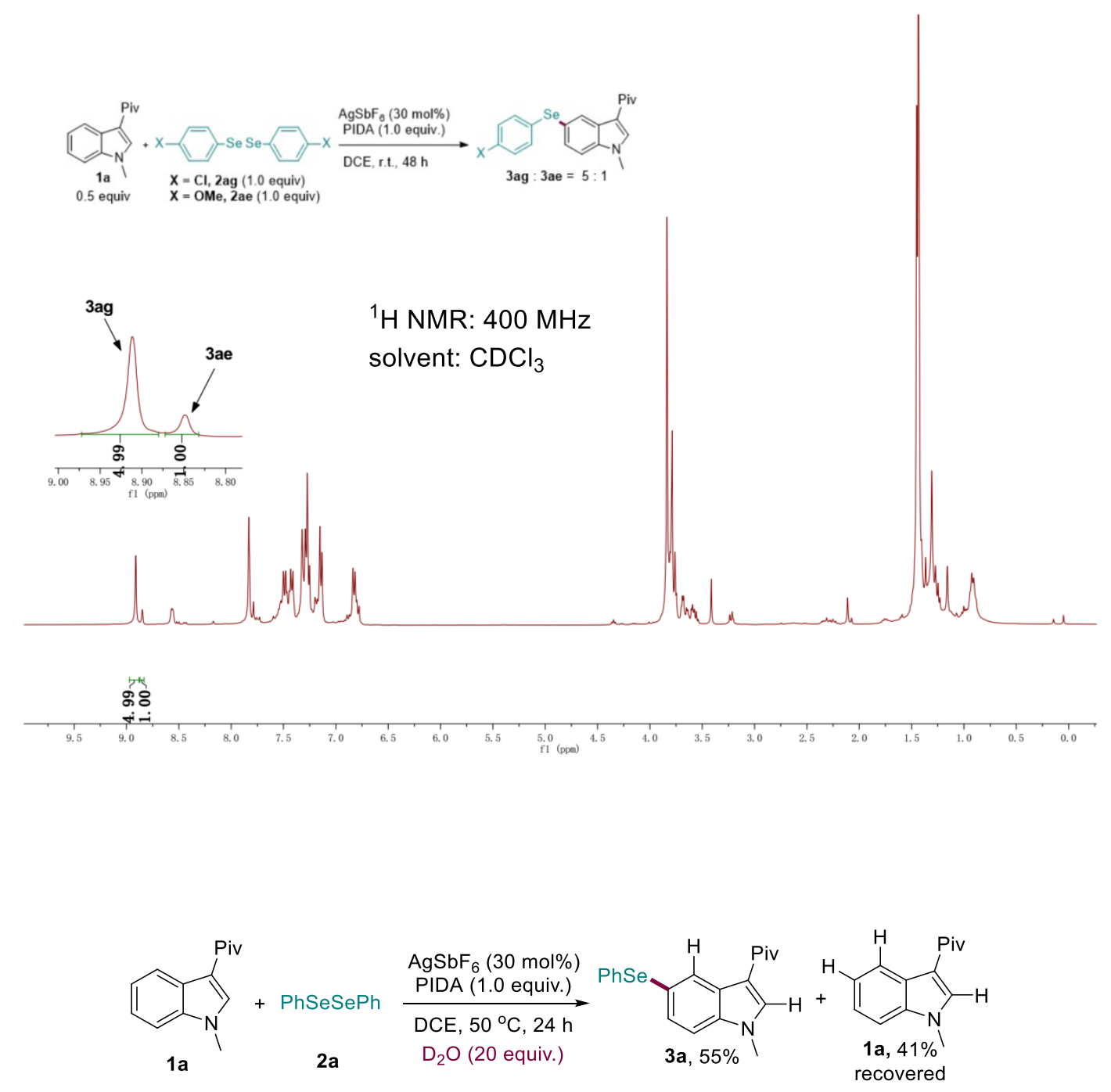

Compound 1a (43.1 mg, $0.2 \mathrm{mmol}), 2 \mathrm{a}$ (34.3 mg, 0.55 equiv.), AgSbF 6 (20.6 mg, 30 mol \%), PIDA (64.4 mg, 0.2 mmol), $\mathrm{D}_{2} \mathrm{O}(80 \mathrm{mg}, 20 \mathrm{mmol})$, and DCE ( $2 \mathrm{~mL}$ ) were charged into a Schlenk tube under $\mathrm{N}_{2}$ atmosphere. The result mixture was stirred at $50{ }^{\circ} \mathrm{C}$ for $24 \mathrm{~h}$. After reaction, recovered 1a and resulted 3a were isolated through silica gel column. ${ }^{1} \mathrm{H}$ NMR analysis of 1a and 3a indicated that no incorporated deuterium was observed. 
Reaction Kinetics:
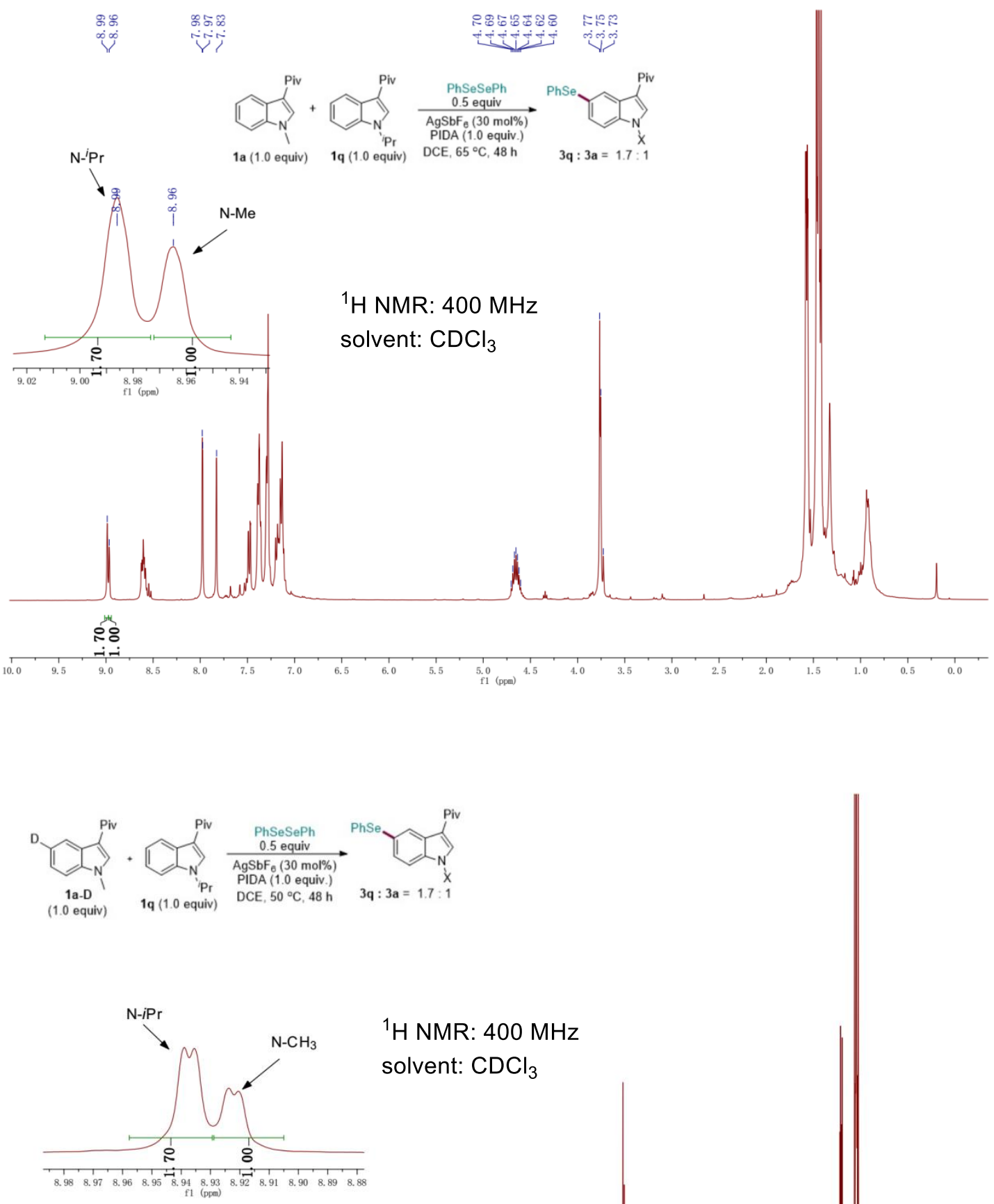

${ }^{1} \mathrm{H}$ NMR: $400 \mathrm{MHz}$ solvent: $\mathrm{CDCl}_{3}$

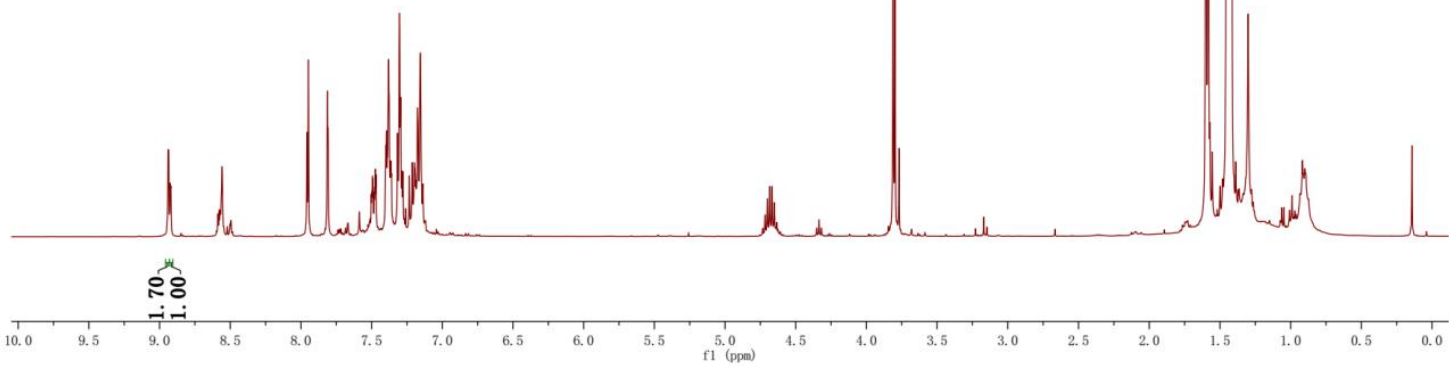




\section{Crystal Data and Structure Refinement of 3n'and 3ag}

Single crystals of $\mathbf{3} \mathbf{n}^{\prime}$ and $\mathbf{3 a g}$ suitable for $\mathrm{x}$-ray analysis were obtained by recrystallization in dichloromethane/hexane at room temperature. Data from a $\mathrm{Cu}$ radiation source were collected in a nitrogen gas stream at $167 \mathrm{~K}$ using phi and omega scans.

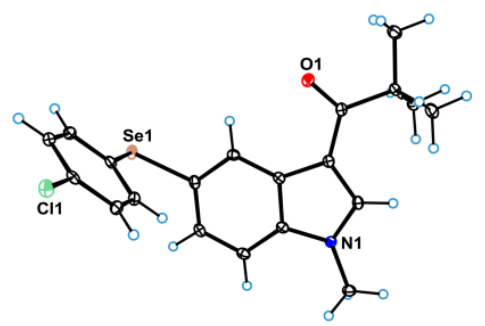

Figure S1. ORTEP representation of 3ag. Thermal ellipsoids are drawn at the $30 \%$ probability level.

\begin{tabular}{|c|c|}
\hline Empirical formula & $\mathrm{C}_{20} \mathrm{H}_{20} \mathrm{ClNOSe}$ \\
\hline Formula weight & 404.78 \\
\hline Temperature / K & $110.10(10)$ \\
\hline Crystal system & monoclinic \\
\hline Space group & $\mathrm{P} 2{ }_{1} / \mathrm{n}$ \\
\hline $\mathrm{a} / \AA ⿻ \mathrm{~b} / \AA, \mathrm{c} / \AA$ & $5.6868(2), 14.0452(10), 22.1678(7)$ \\
\hline$\alpha /{ }^{\circ}, \beta /{ }^{\circ}, \gamma /{ }^{\circ}$ & $90.00,96.970(3), 90.00$ \\
\hline Volume / $\AA^{3}$ & $1757.51(15)$ \\
\hline $\mathrm{Z}$ & 4 \\
\hline$\rho_{\text {calc }} / \mathrm{mg} \mathrm{mm}^{-3}$ & 1.530 \\
\hline$\mu / \mathrm{mm}^{-1}$ & 2.295 \\
\hline $\mathrm{F}(000)$ & 824 \\
\hline Crystal size $/ \mathrm{mm}^{3}$ & $0.40 \times 0.37 \times 0.03$ \\
\hline $2 \Theta$ range for data collection & 6.88 to $52^{\circ}$ \\
\hline Index ranges & $-7 \leq \mathrm{h} \leq 6,-17 \leq \mathrm{k} \leq 12,-20 \leq 1 \leq 27$ \\
\hline Reflections collected & 7939 \\
\hline Independent reflections & $3434[\mathrm{R}(\mathrm{int})=0.0332($ inf- $0.9 \AA)]$ \\
\hline Data/restraints/parameters & $3434 / 0 / 221$ \\
\hline Goodness-of-fit on $\mathrm{F}^{2}$ & 1.042 \\
\hline \multicolumn{2}{|c|}{ Final $R$ indexes $\left[\mathrm{I}>2 \sigma(\mathrm{I})\right.$ i.e. $\left.\mathrm{F}_{\mathrm{o}}>4 \sigma\left(\mathrm{F}_{\mathrm{o}}\right)\right] \mathrm{R}_{1}=0.0348, \mathrm{wR}_{2}=0.0713$} \\
\hline Final $\mathrm{R}$ indexes [all data] & $\mathrm{R}_{1}=0.0446, \mathrm{wR}_{2}=0.0760$ \\
\hline Largest diff. peak/hole / e $\AA^{-3}$ & $0.599 /-0.418$ \\
\hline Flack Parameters & $\mathrm{N}$ \\
\hline Completeness & 0.9955 \\
\hline
\end{tabular}




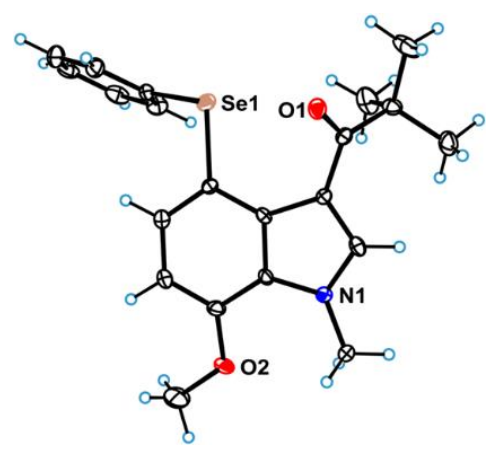

Figure S2. ORTEP representation of $\mathbf{3 n}$. Thermal ellipsoids are drawn at the $30 \%$ probability level.

Identification code

Empirical formula

Formula weight

Temperature / K

Crystal system

Space group

$\mathrm{a} / \AA, \mathrm{b} / \AA, \mathrm{c} / \AA$

$\alpha /^{\circ}, \beta /{ }^{\circ}, \gamma /{ }^{\circ}$

Volume / $\AA^{3}$

Z

$\rho_{\text {calc }} / \mathrm{mg} \mathrm{mm}^{-3}$

$\mu / \mathrm{mm}^{-1}$

$\mathrm{F}(000)$

Crystal size $/ \mathrm{mm}^{3}$

$2 \Theta$ range for data collection

Index ranges

Reflections collected

Independent reflections

Data/restraints/parameters

Goodness-of-fit on $\mathrm{F}^{2}$ exp_5750

$\mathrm{C}_{21} \mathrm{H}_{23} \mathrm{NO}_{2} \mathrm{Se}$

400.36

146(2)

orthorhombic

Pna2 1

$12.0693(8), 10.9616(8), 29.0524(19)$

$90.00,90.00,90.00$

3843.6(5)

8

1.384

1.967

1648

$0.40 \times 0.35 \times 0.15$

6.56 to $52^{\circ}$

$-14 \leq \mathrm{h} \leq 14,-13 \leq \mathrm{k} \leq 8,-35 \leq 1 \leq 30$

10925

$6109[\mathrm{R}(\mathrm{int})=0.0441(\mathrm{inf}-0.9 \AA)]$

$6109 / 1 / 462$

1.030

Final $R$ indexes $\left[\mathrm{I}>2 \sigma(\mathrm{I})\right.$ i.e. $\left.\mathrm{F}_{\mathrm{o}}>4 \sigma\left(\mathrm{F}_{\mathrm{o}}\right)\right] \mathrm{R}_{1}=0.0471, \mathrm{wR}_{2}=0.0820$

Final R indexes [all data]

$\mathrm{R}_{1}=0.0677, \mathrm{wR}_{2}=0.0911$

Largest diff. peak/hole / e $\AA^{-3}$

$0.609 /-0.445$

Flack Parameters

$0.000(18)$

Completeness

0.9962 

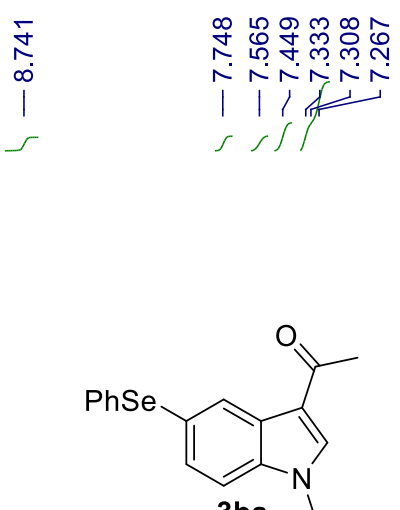

${ }^{1} \mathrm{H}$ NMR: $400 \mathrm{MHz}$

3 ba

solvent: $\mathrm{CDCl}_{3}$

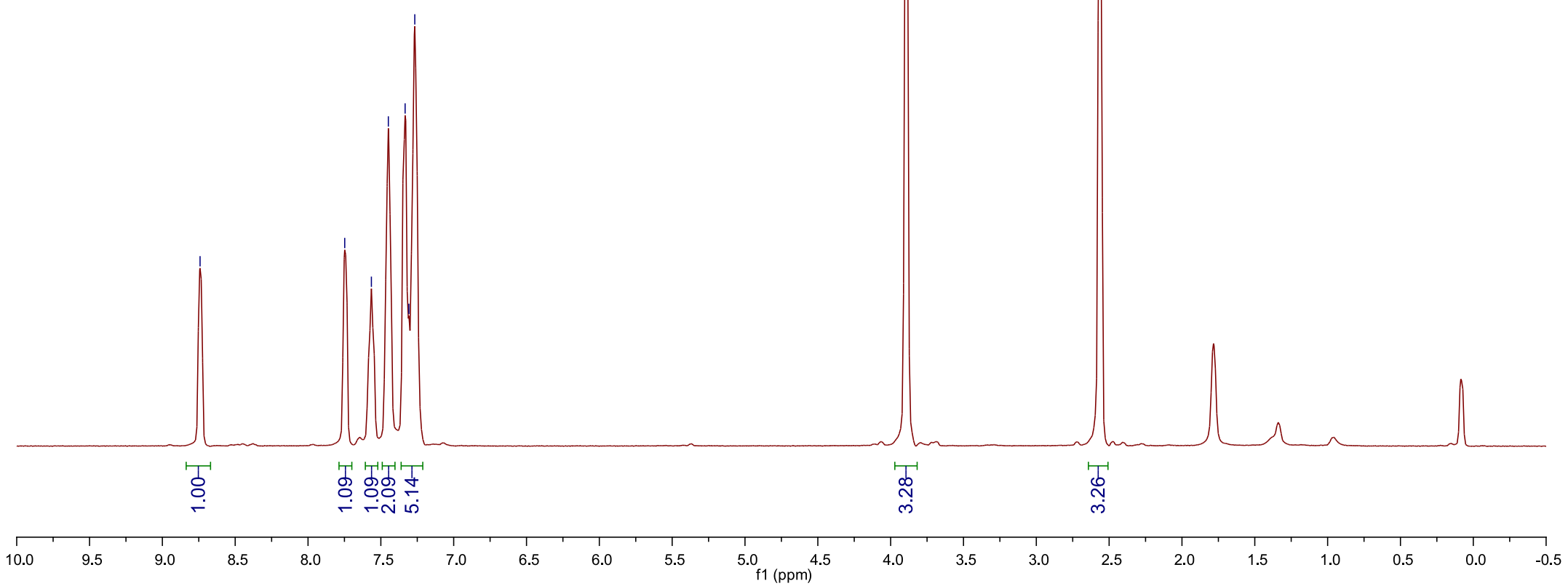




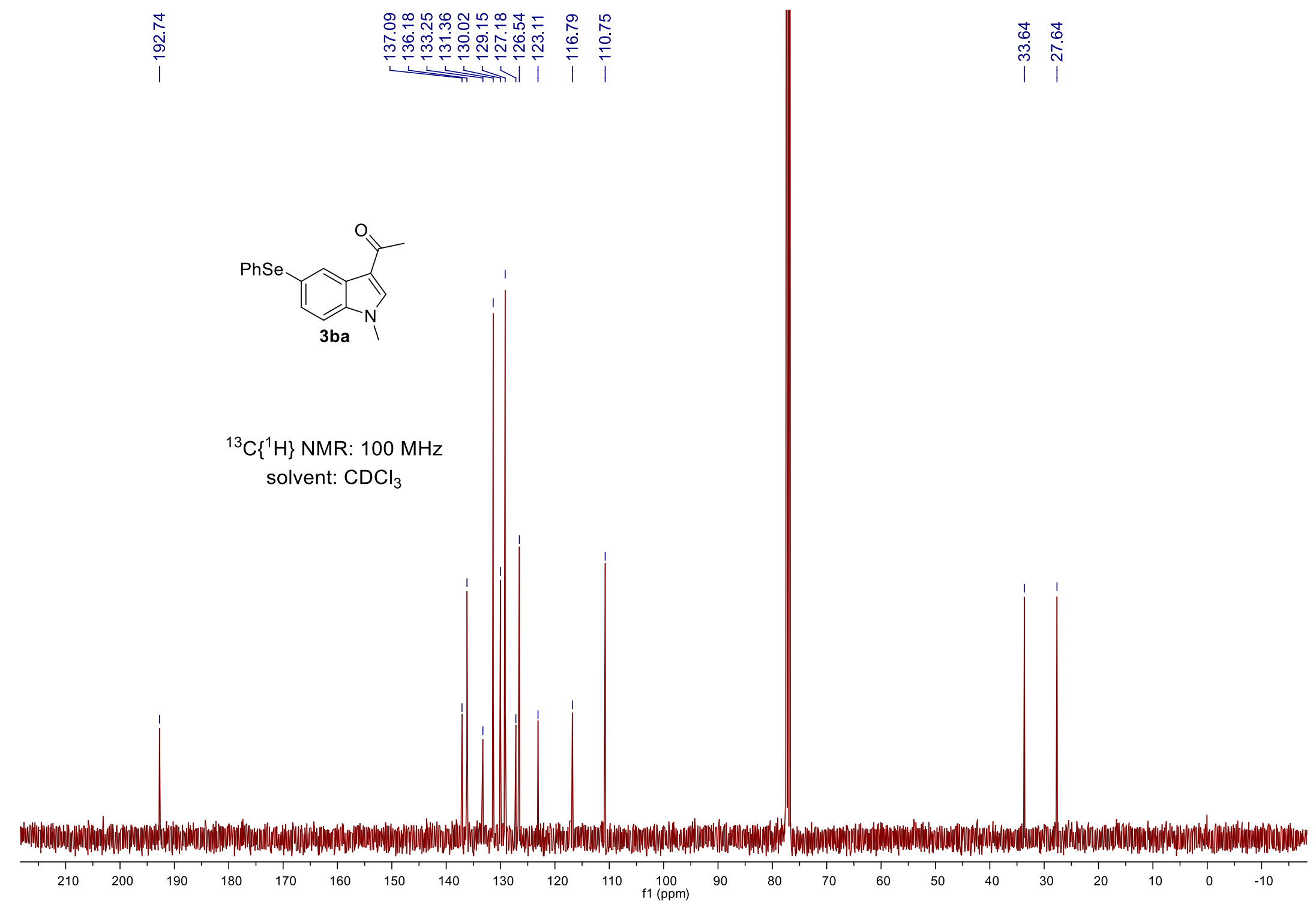




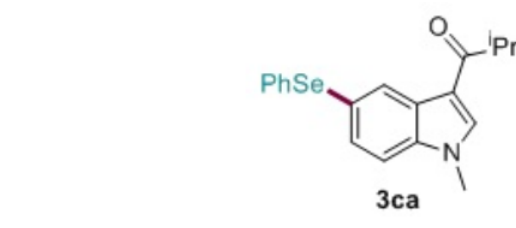

${ }^{1} \mathrm{H}$ NMR: $400 \mathrm{MHz}$

solvent: $\mathrm{CDCl}_{3}$

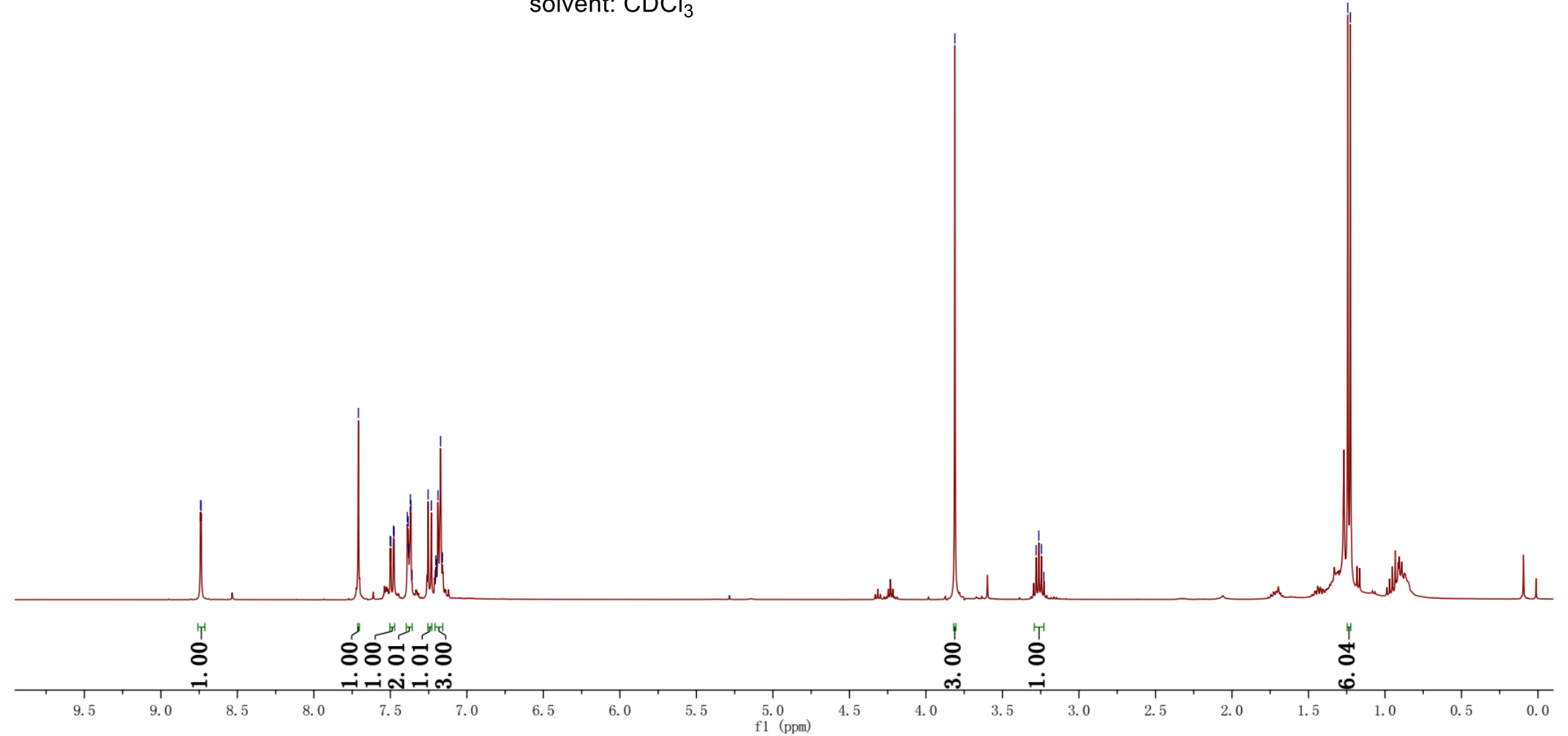




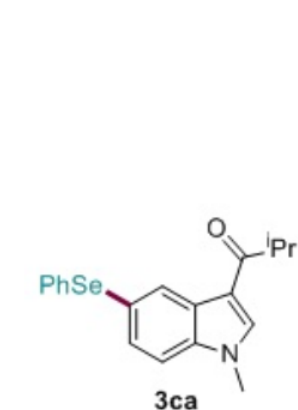

\section{${ }^{13} \mathrm{C}\left\{{ }^{1} \mathrm{H}\right\}$ NMR: $100 \mathrm{MHz}$} solvent: $\mathrm{CDCl}_{3}$
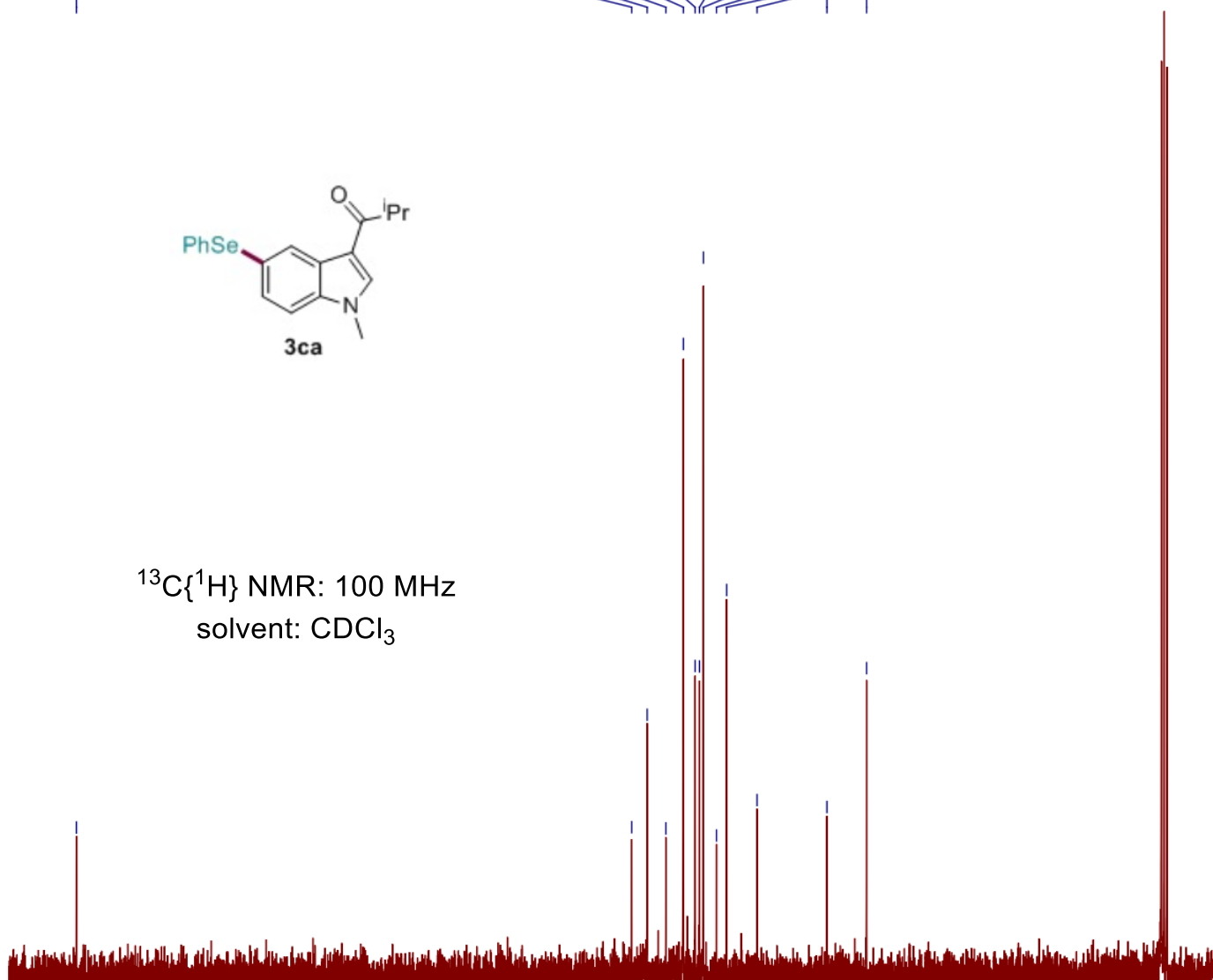

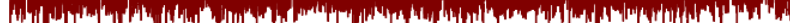

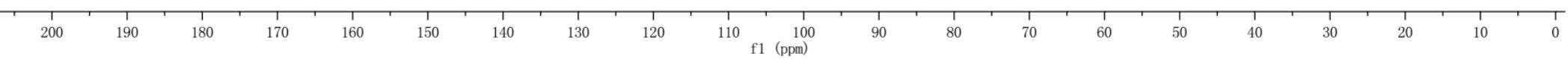




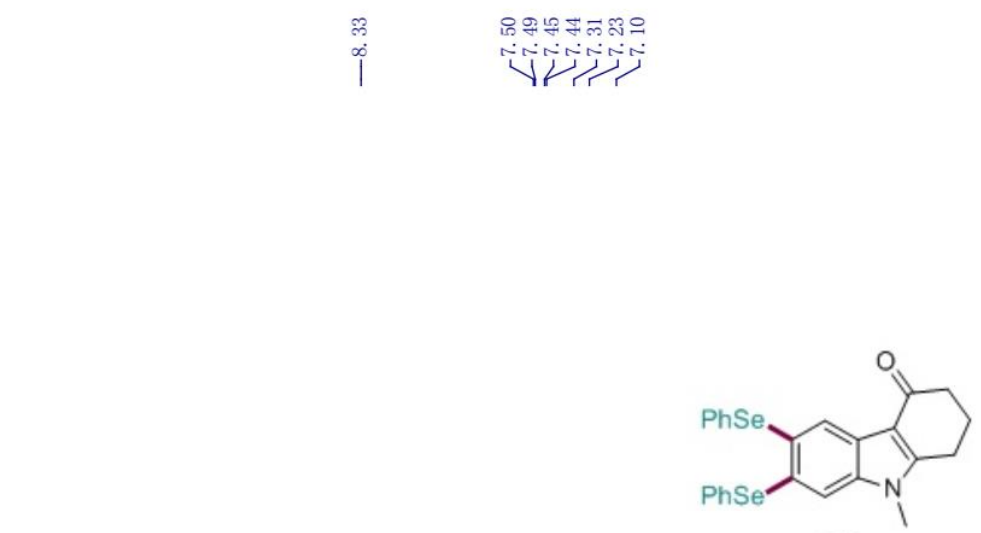

3da

${ }^{1} \mathrm{H}$ NMR: $400 \mathrm{MHz}$

solvent: $\mathrm{CDCl}_{3}$

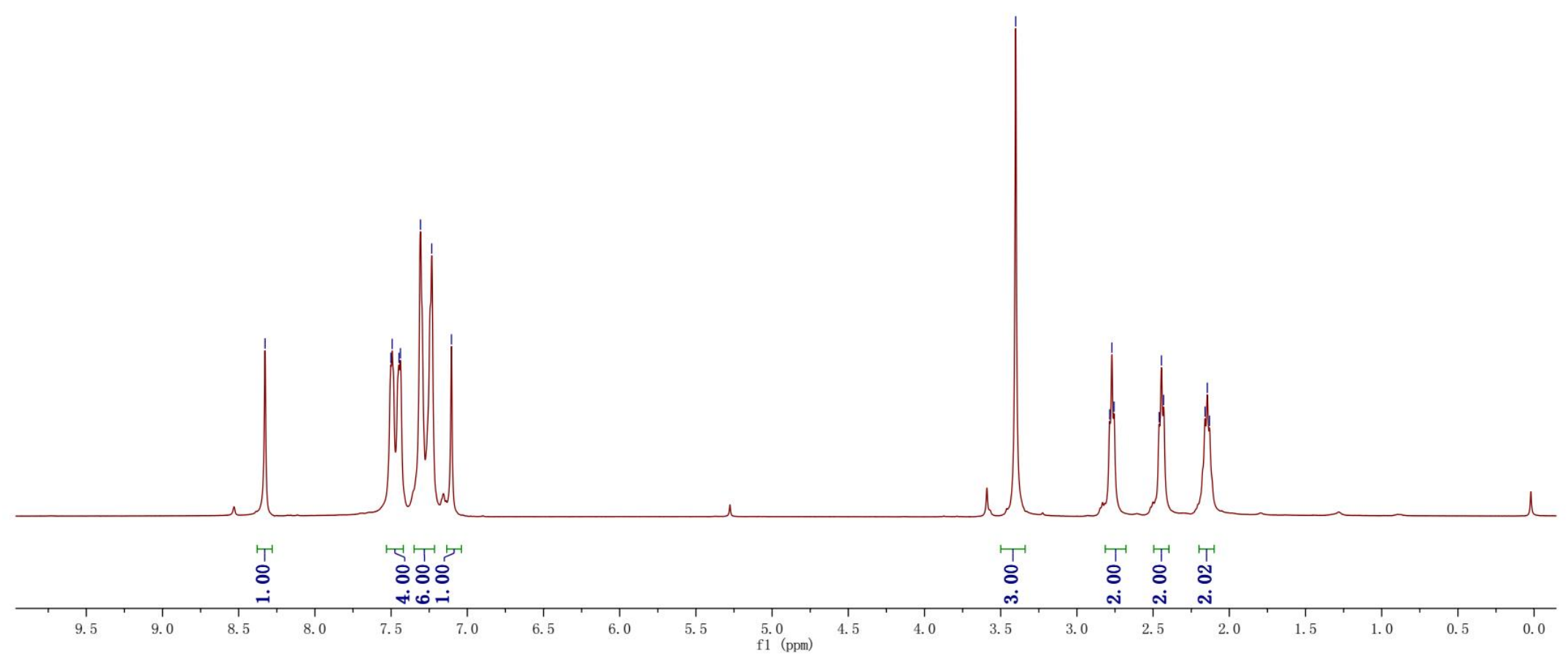




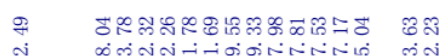

|

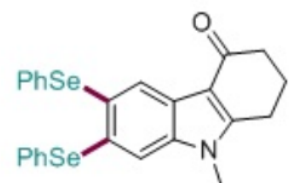

3da

${ }^{13} \mathrm{C}\left\{{ }^{1} \mathrm{H}\right\}$ NMR: $100 \mathrm{MHz}$ solvent: $\mathrm{CDCl}_{3}$

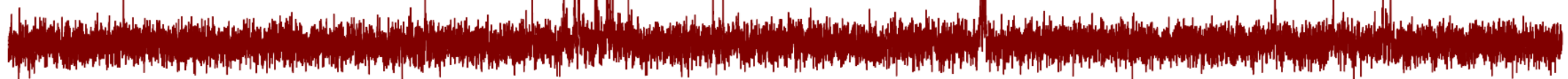

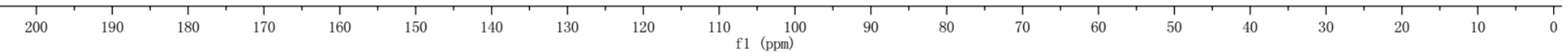


|

3ea

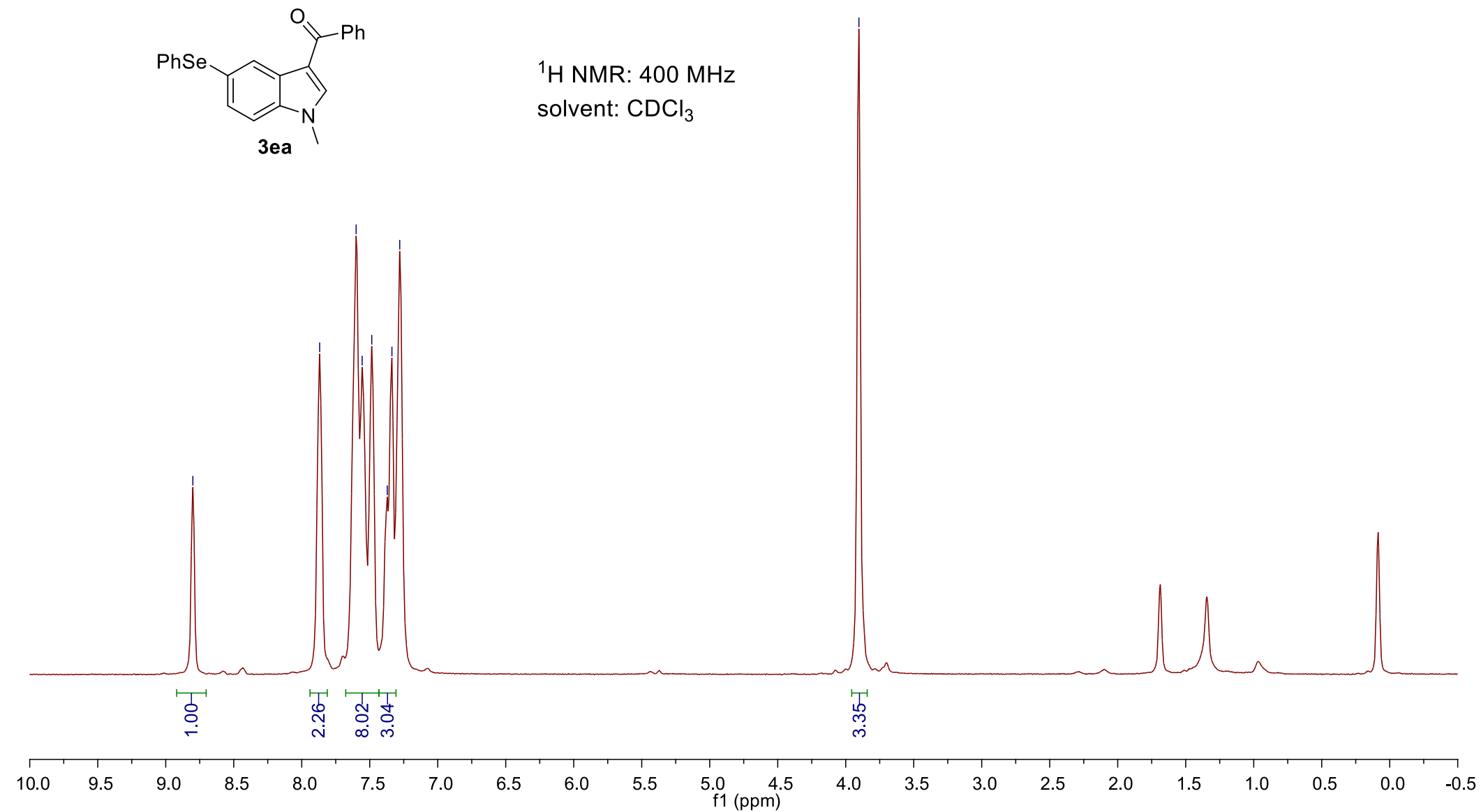




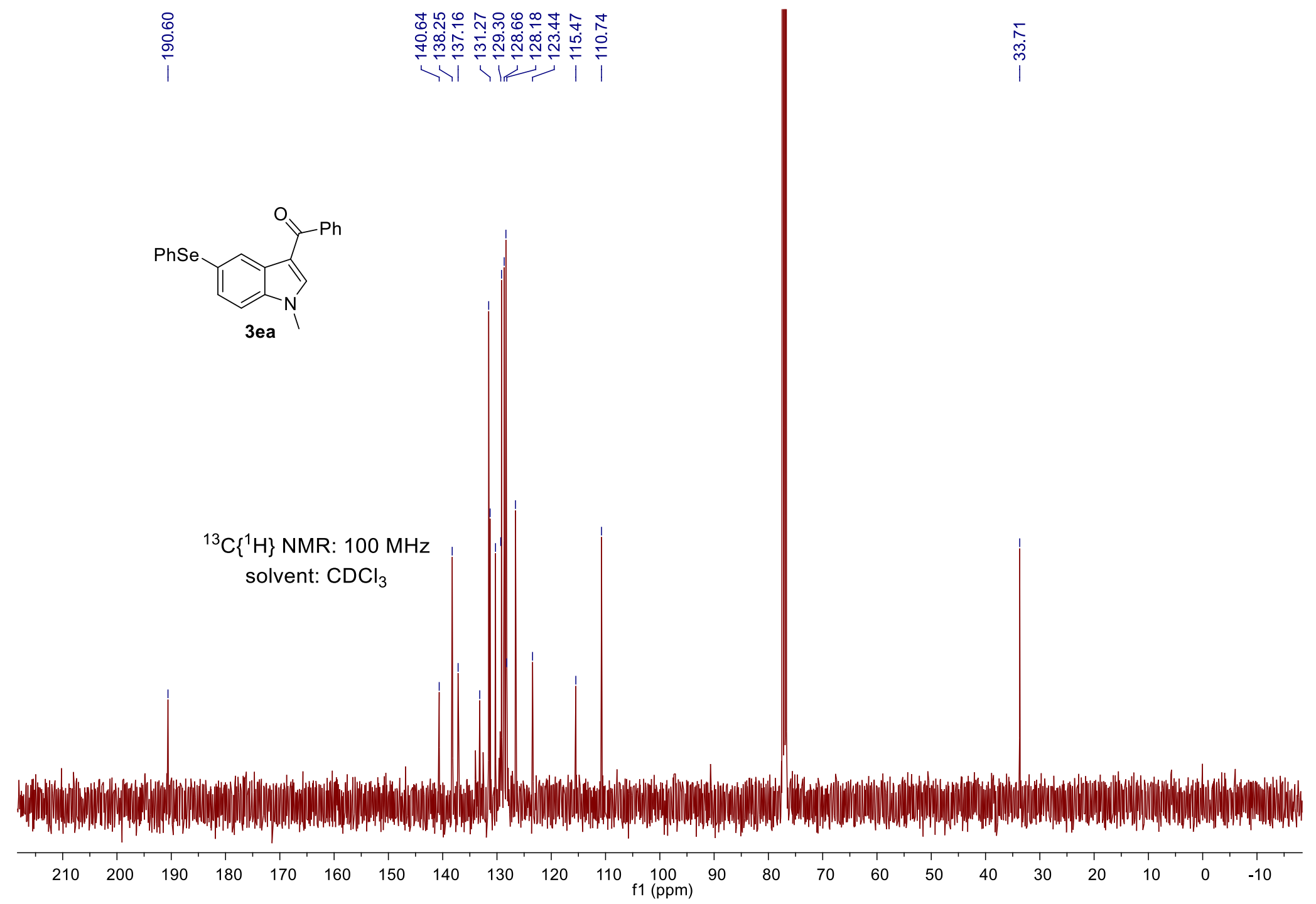




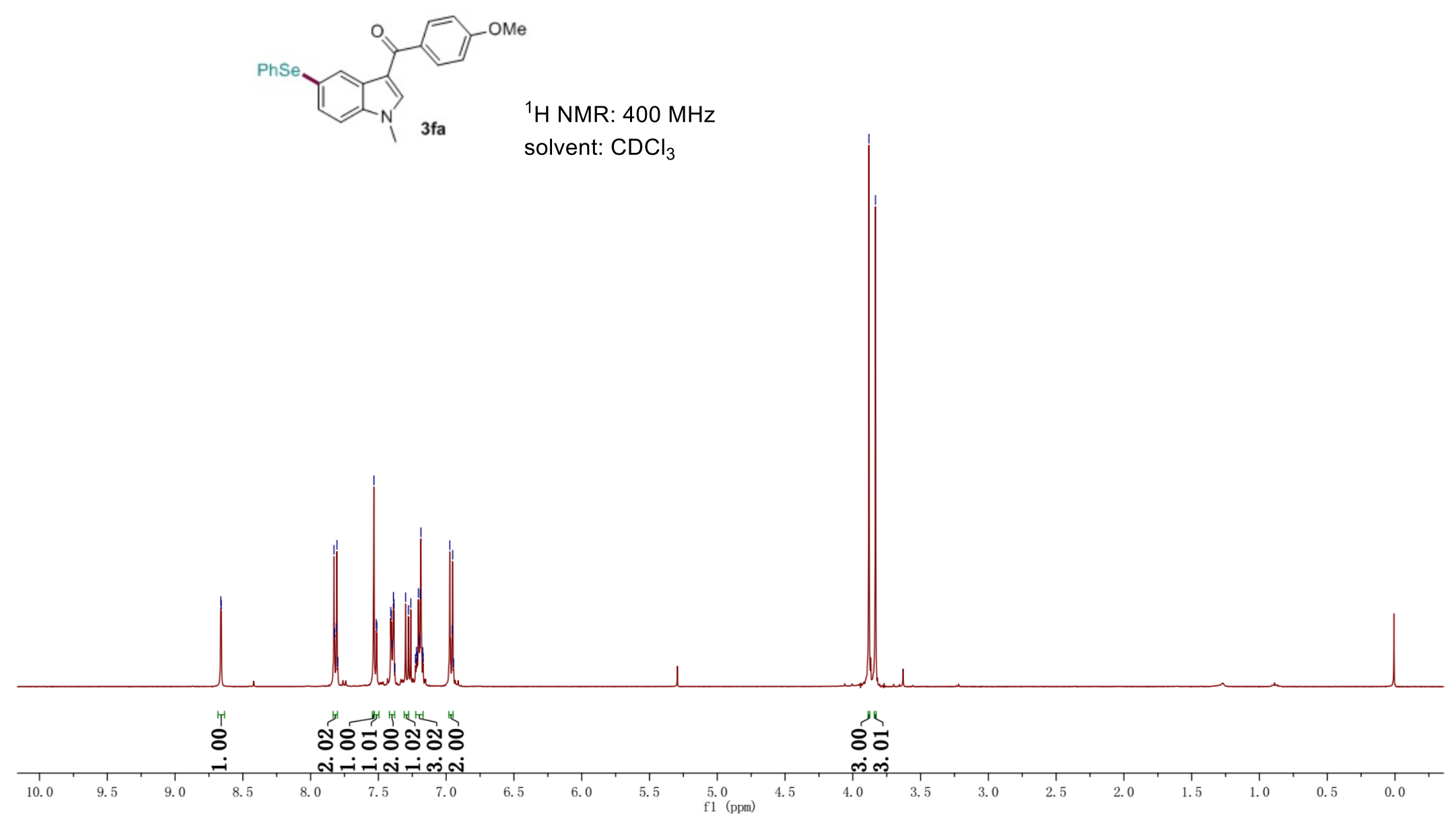




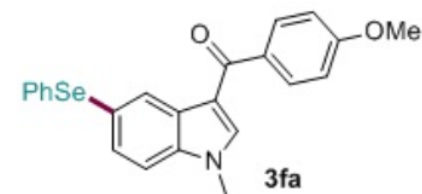

${ }^{13} \mathrm{C}\left\{{ }^{1} \mathrm{H}\right\}$ NMR: $100 \mathrm{MHz}$ solvent: $\mathrm{CDCl}_{3}$
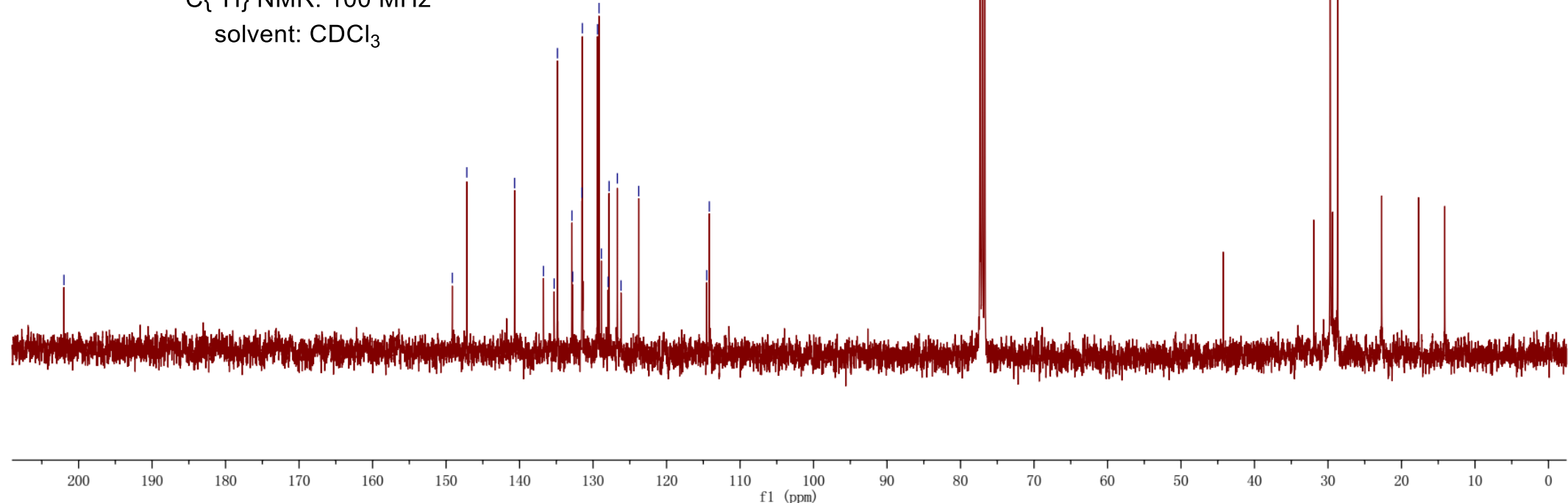


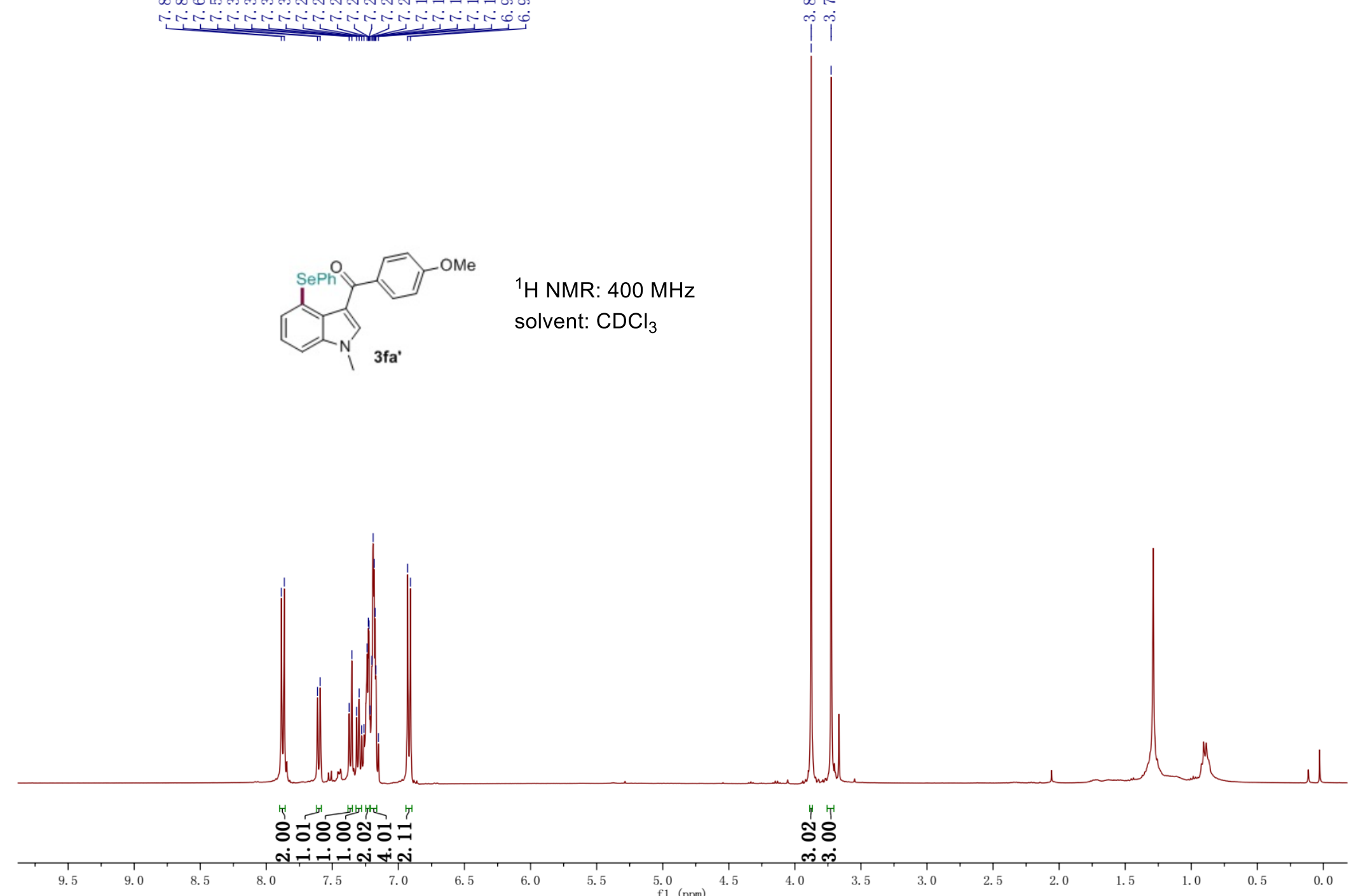




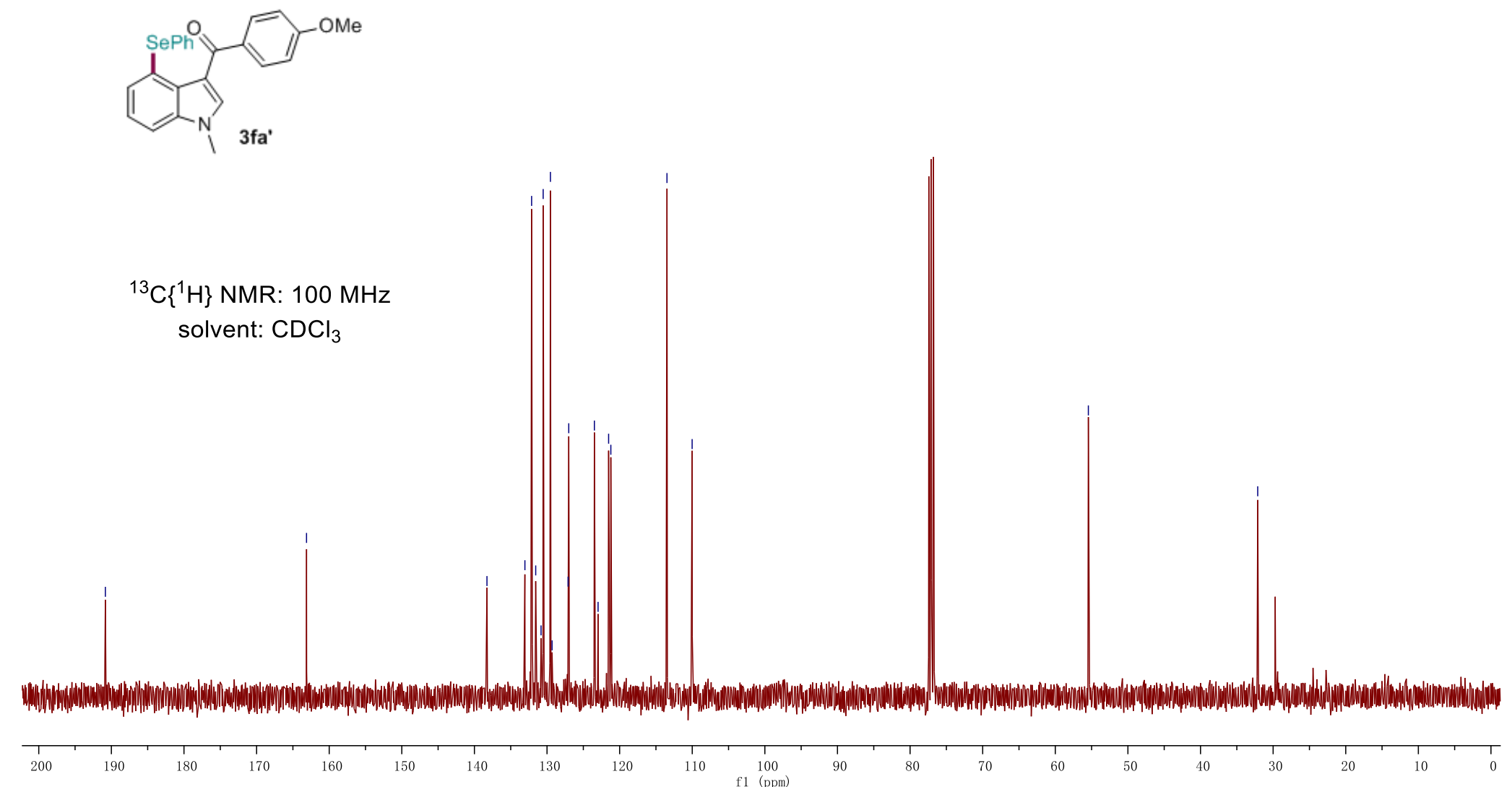




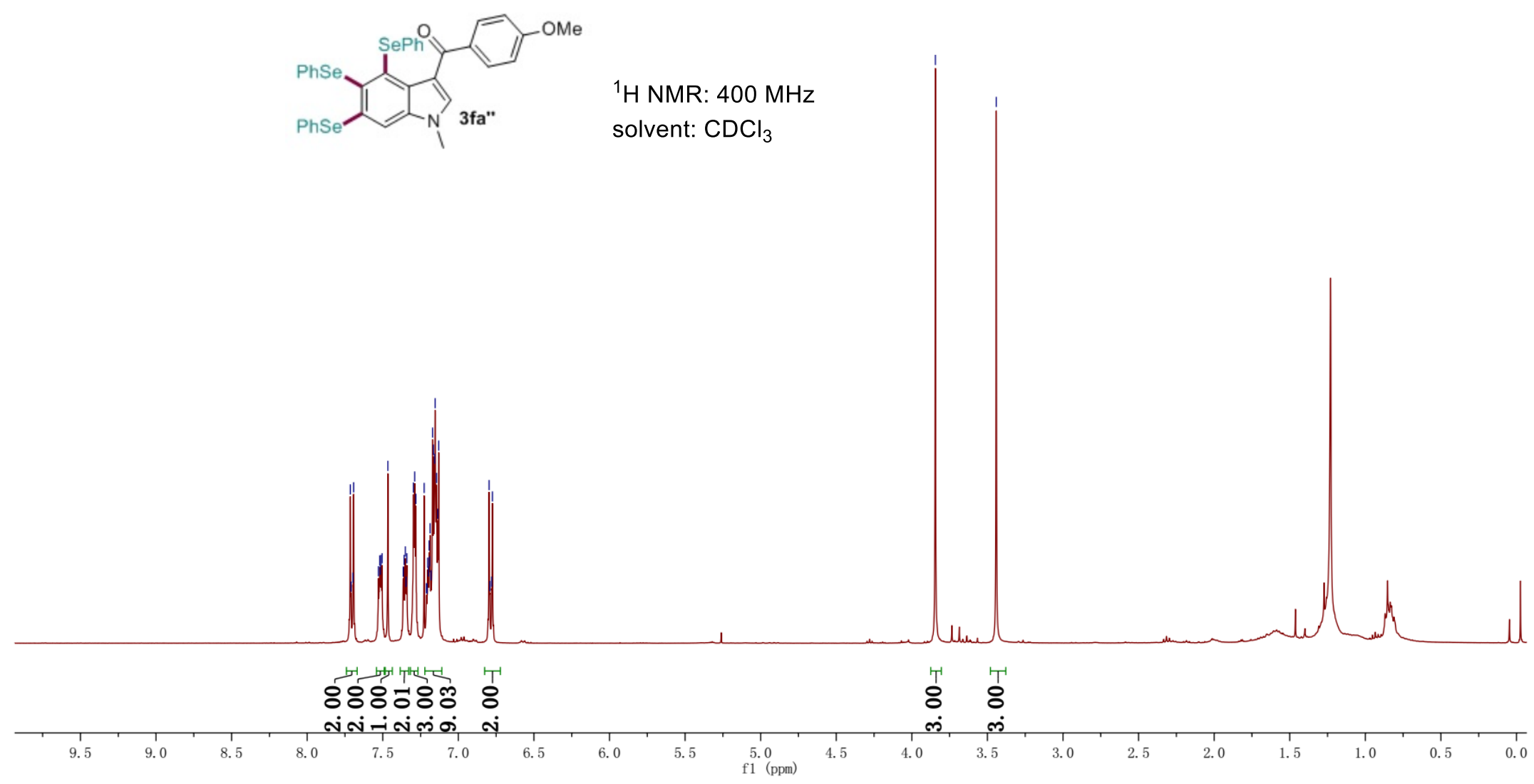




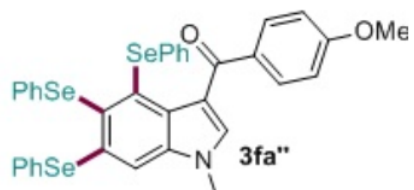

${ }^{13} \mathrm{C}\left\{{ }^{1} \mathrm{H}\right\}$ NMR: $100 \mathrm{MHz}$ solvent: $\mathrm{CDCl}_{3}$

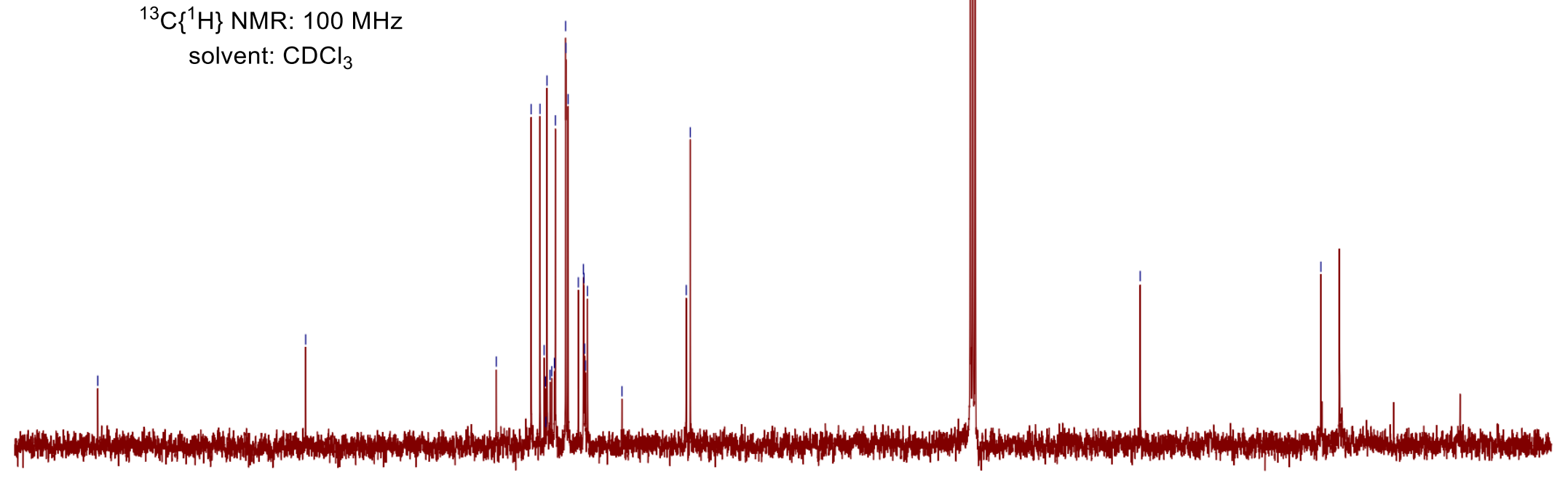




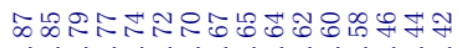

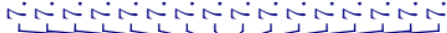

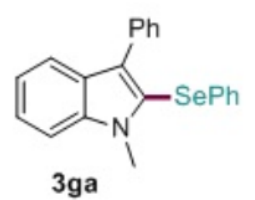

${ }^{1} \mathrm{H}$ NMR: $400 \mathrm{MHz}$

3ga solvent: $\mathrm{CDCl}_{3}$

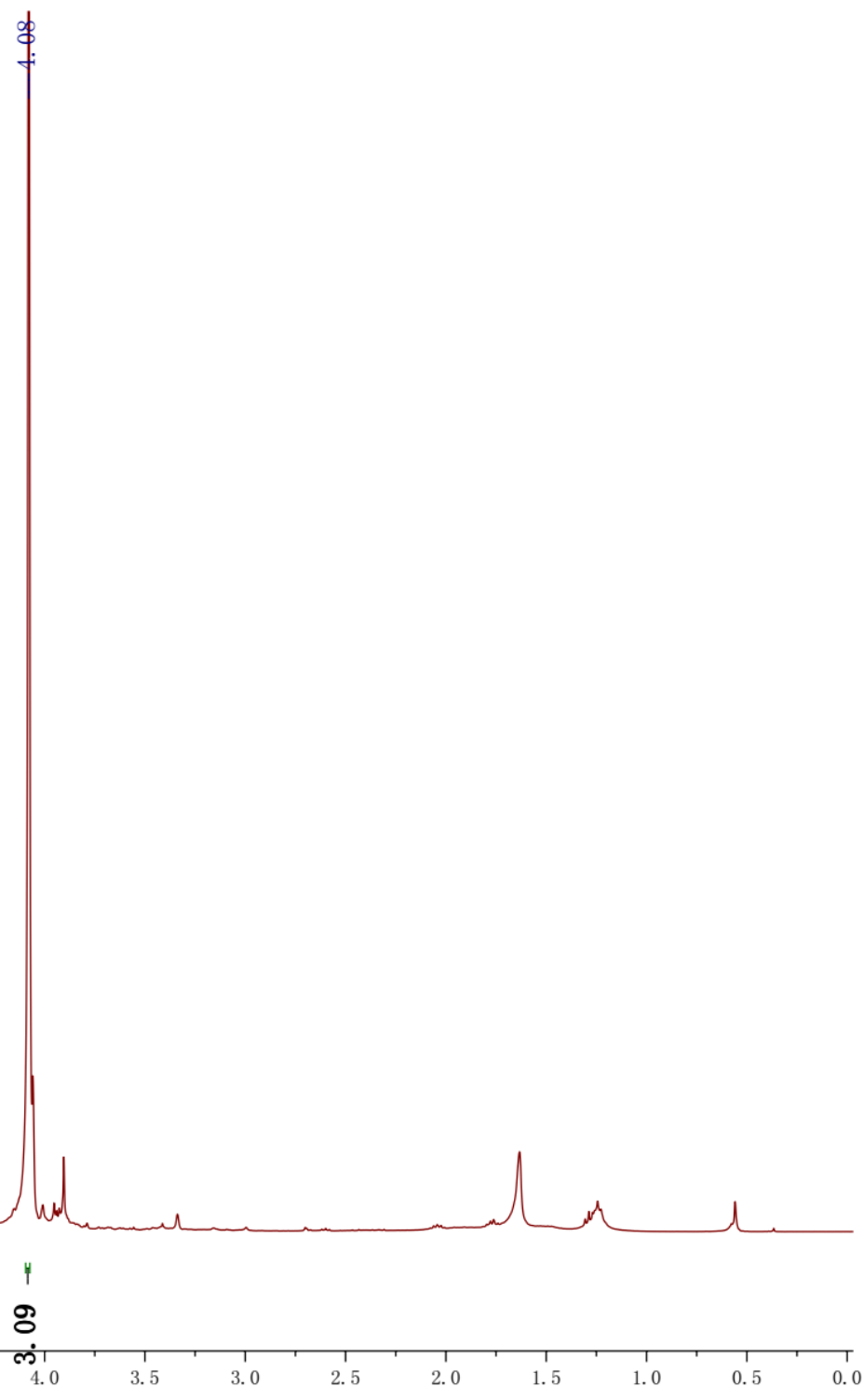



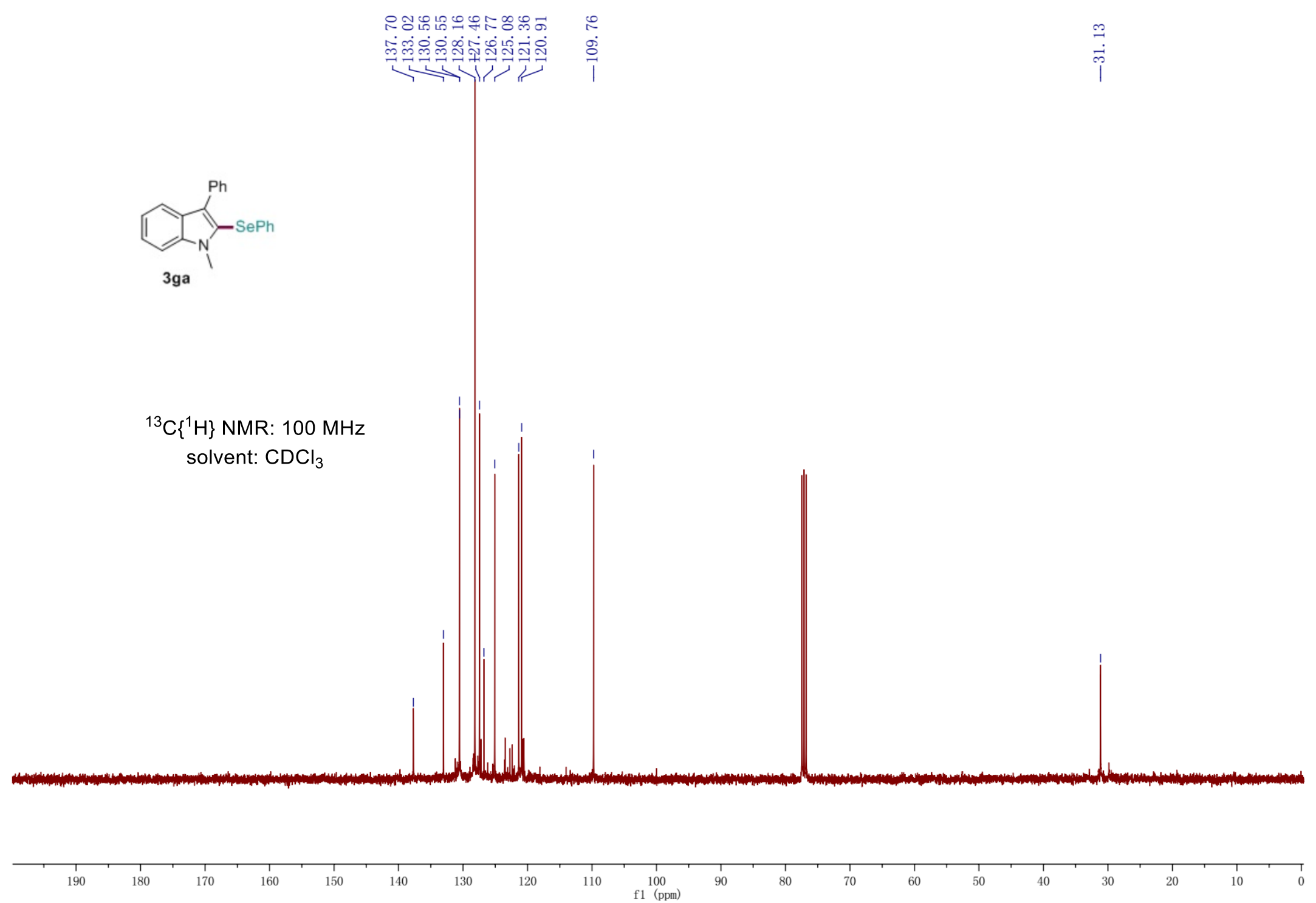
எ எ

NNNNNNNNNRNNNRNAN

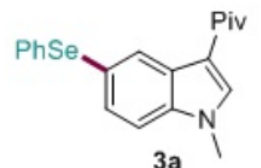

${ }^{1} \mathrm{H}$ NMR: $400 \mathrm{MHz}$

3a

solvent: $\mathrm{CDCl}_{3}$

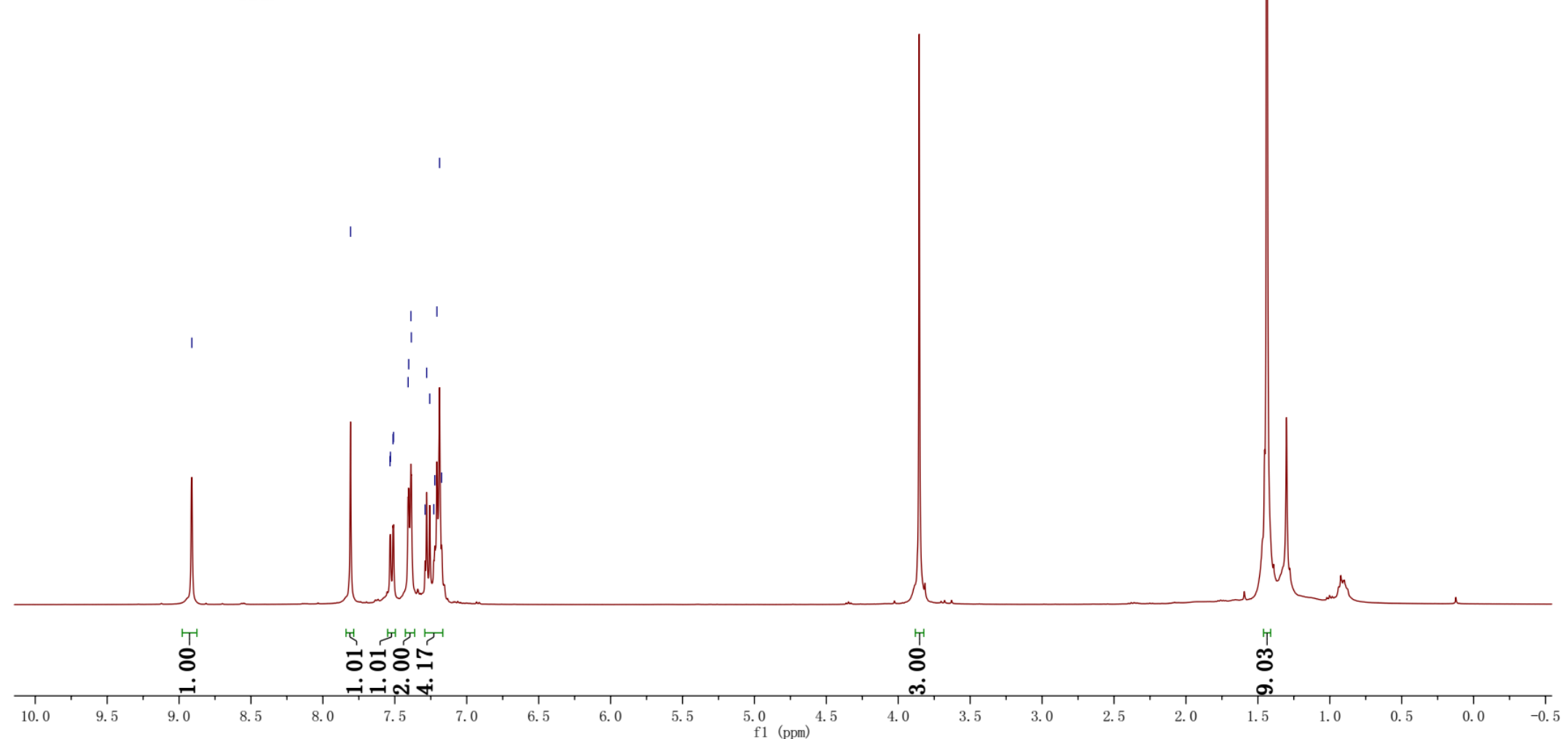




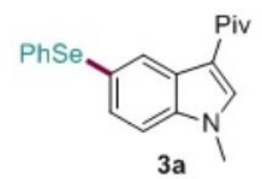

${ }^{13} \mathrm{C}\left\{{ }^{1} \mathrm{H}\right\}$ NMR: $100 \mathrm{MHz}$ solvent: $\mathrm{CDCl}_{3}$
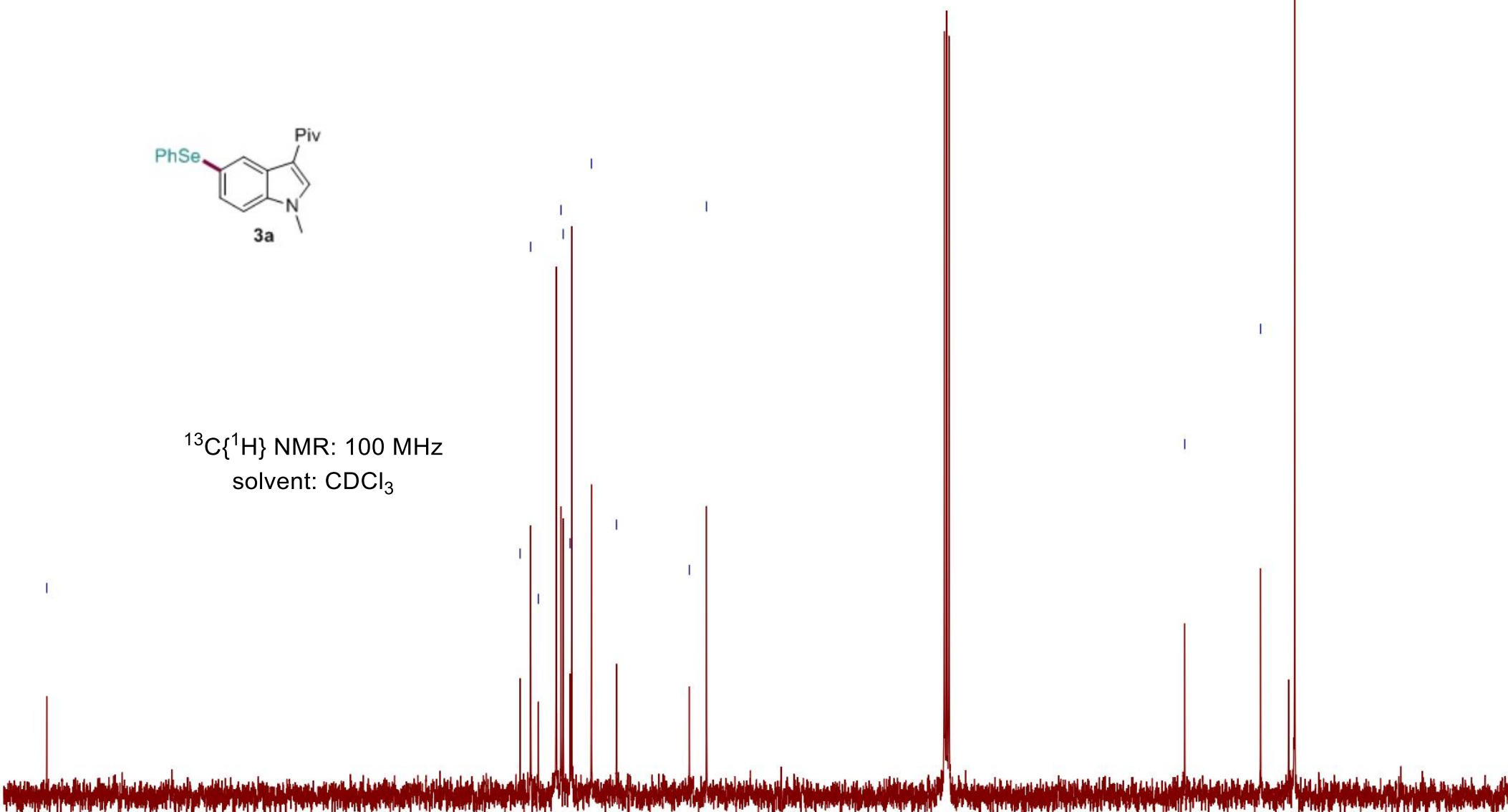

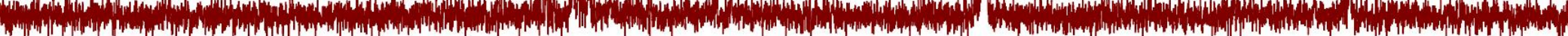

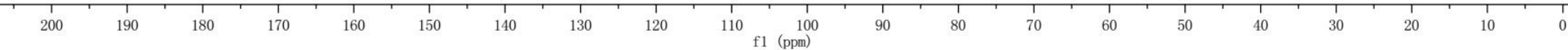




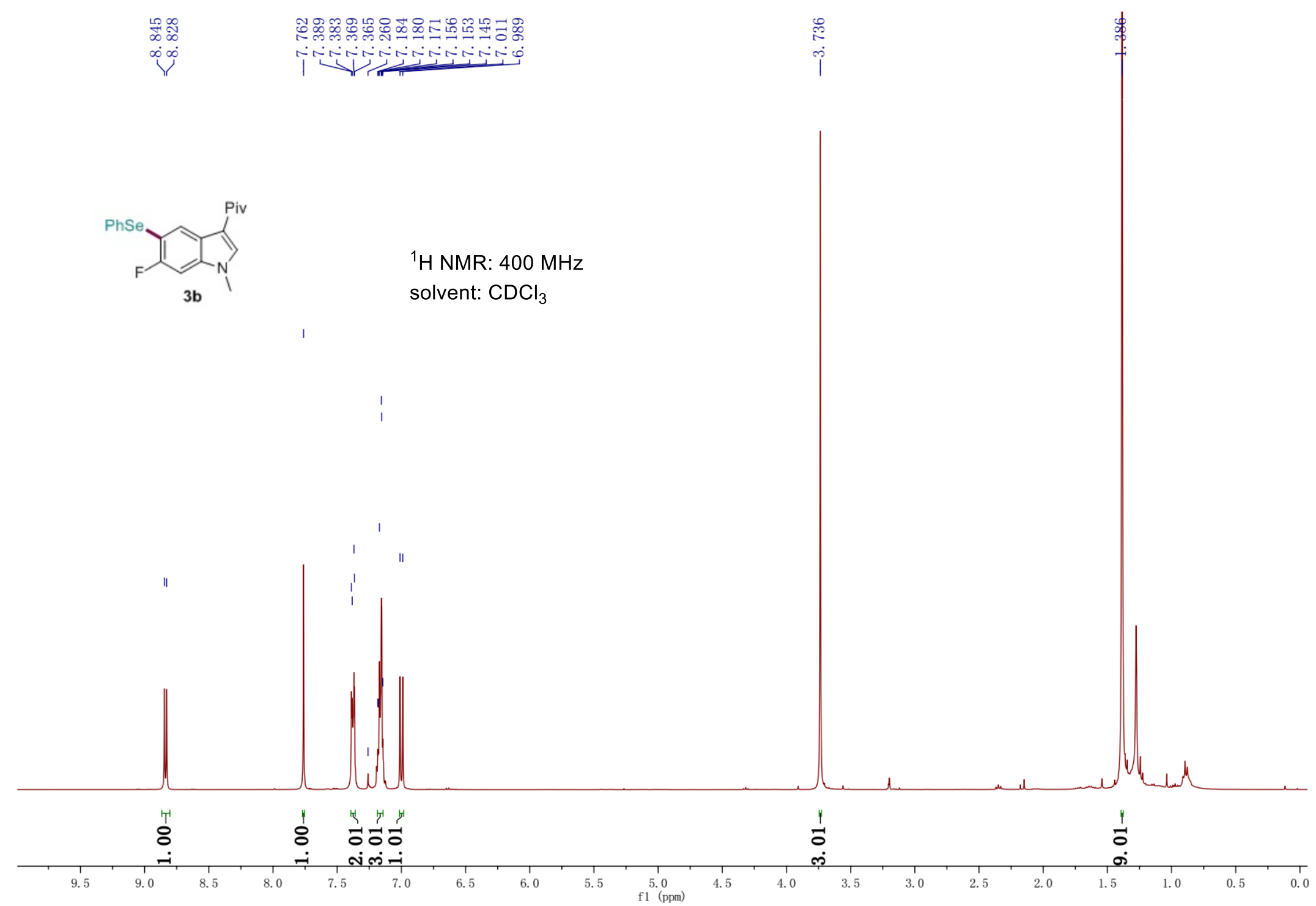




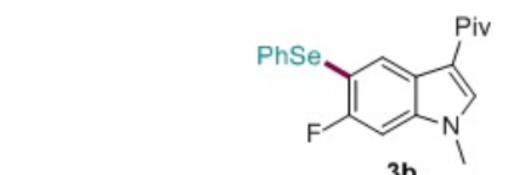

3b

${ }^{3} \mathrm{C}\left\{{ }^{1} \mathrm{H}\right\}$ NMR: $100 \mathrm{MHz}$ solvent: $\mathrm{CDCl}_{3}$

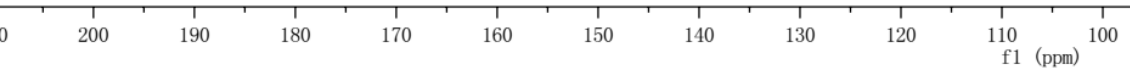




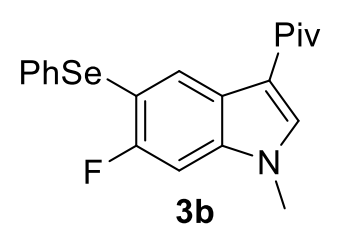

${ }^{19}$ F NMR: $377 \mathrm{MHz}$

Solvent: $\mathrm{CDCl}_{3}$

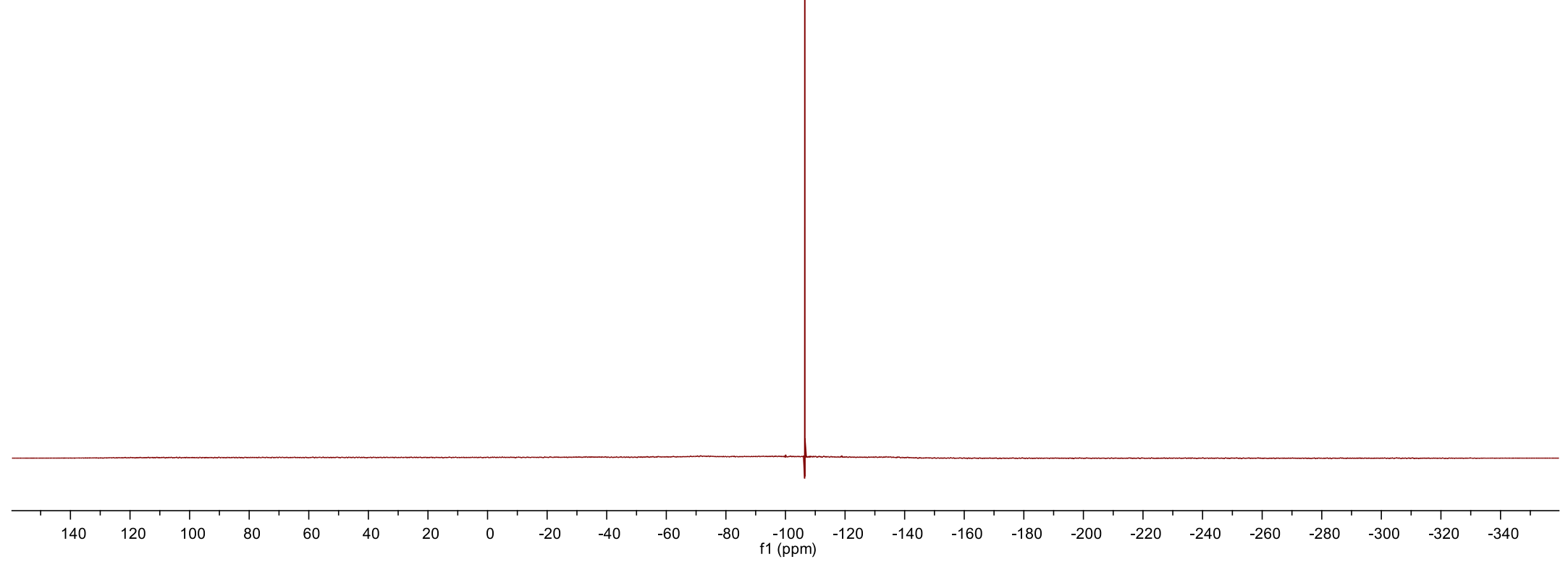




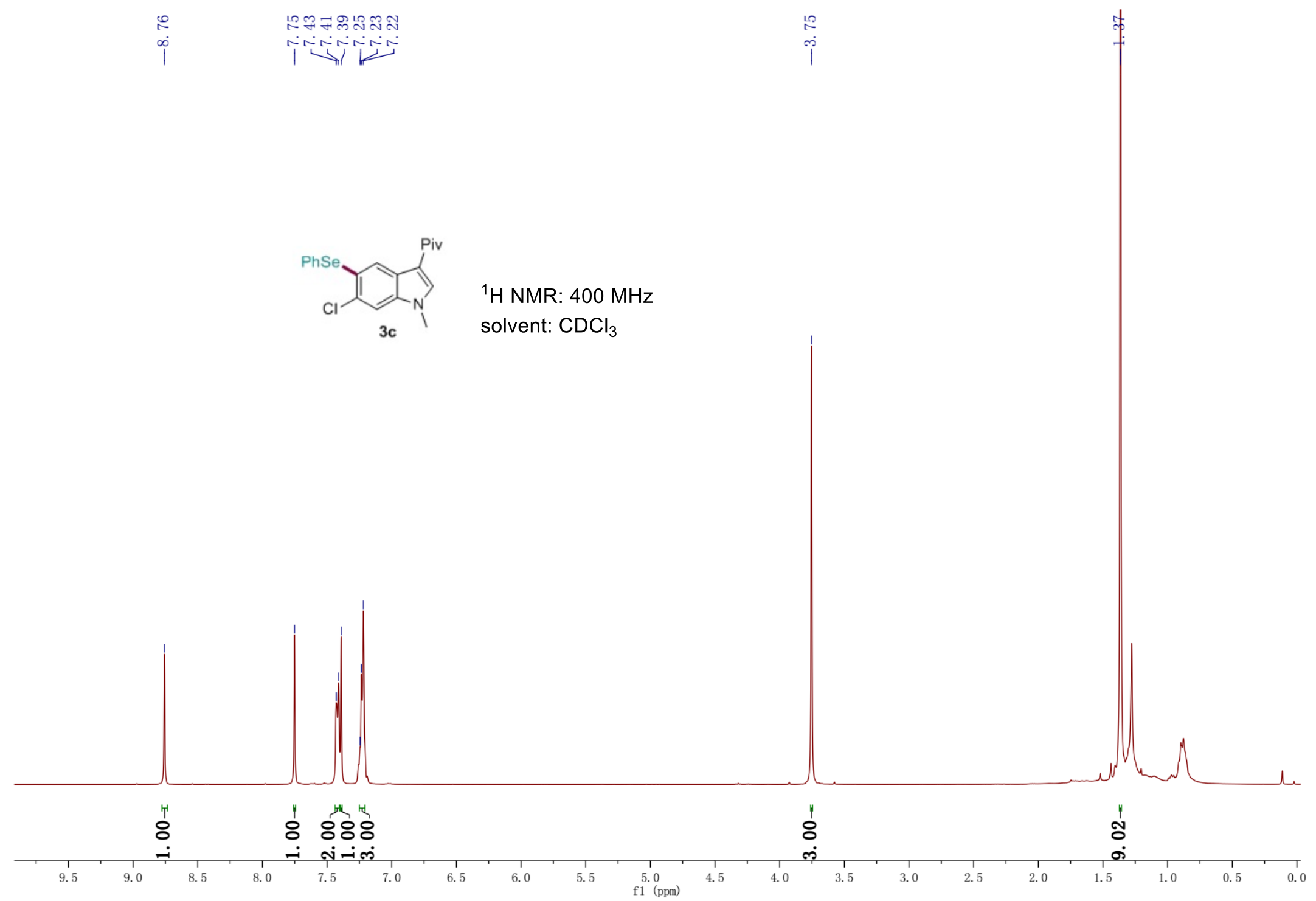




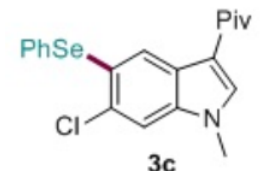

$3 c$

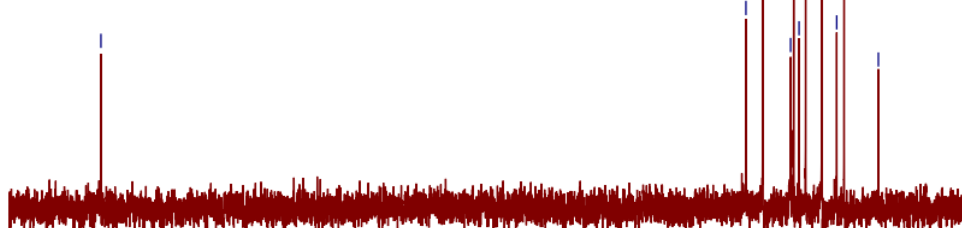

${ }^{13} \mathrm{C}\left\{{ }^{1} \mathrm{H}\right\}$ NMR: $100 \mathrm{MHz}$ solvent: $\mathrm{CDCl}_{3}$ 


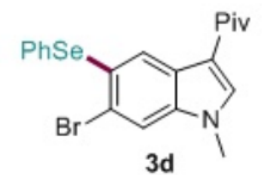

${ }^{1} \mathrm{H}$ NMR: $400 \mathrm{MHz}$

solvent: $\mathrm{CDCl}_{3}$

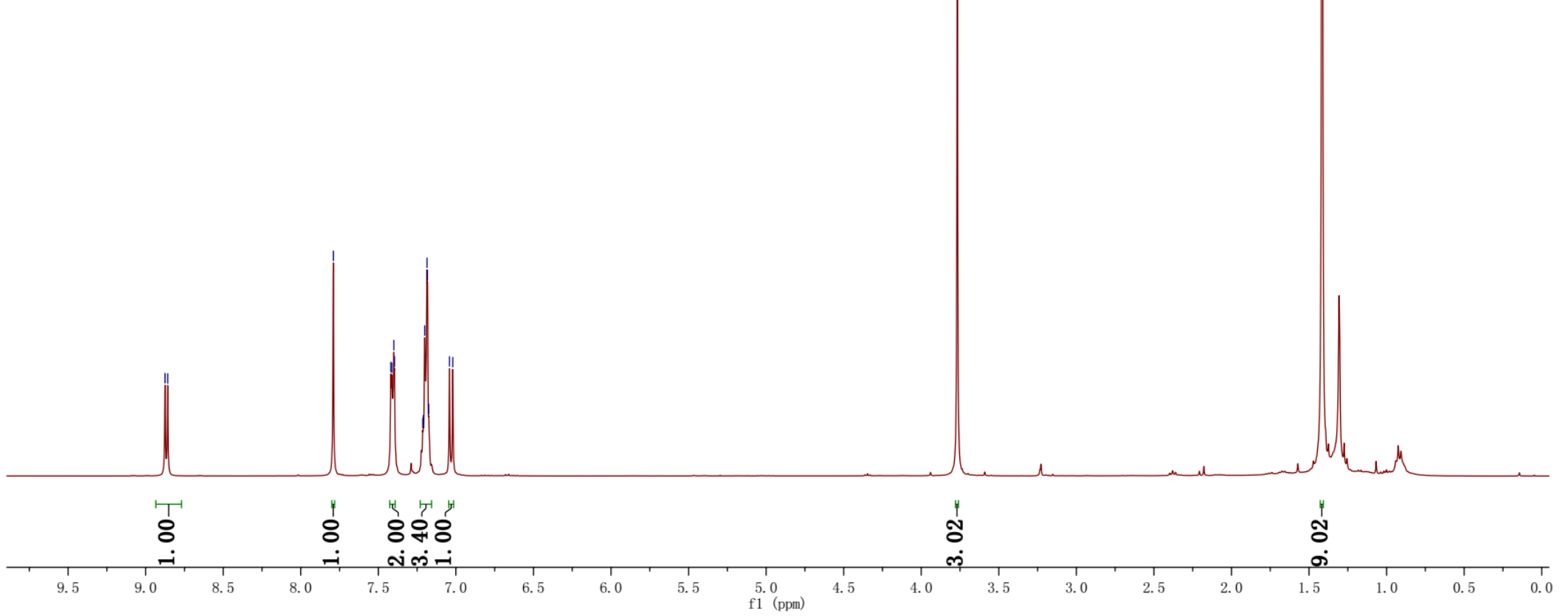




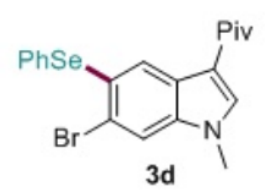

${ }^{13} \mathrm{C}\left\{{ }^{1} \mathrm{H}\right\}$ NMR: $100 \mathrm{MHz}$ solvent: $\mathrm{CDCl}_{3}$
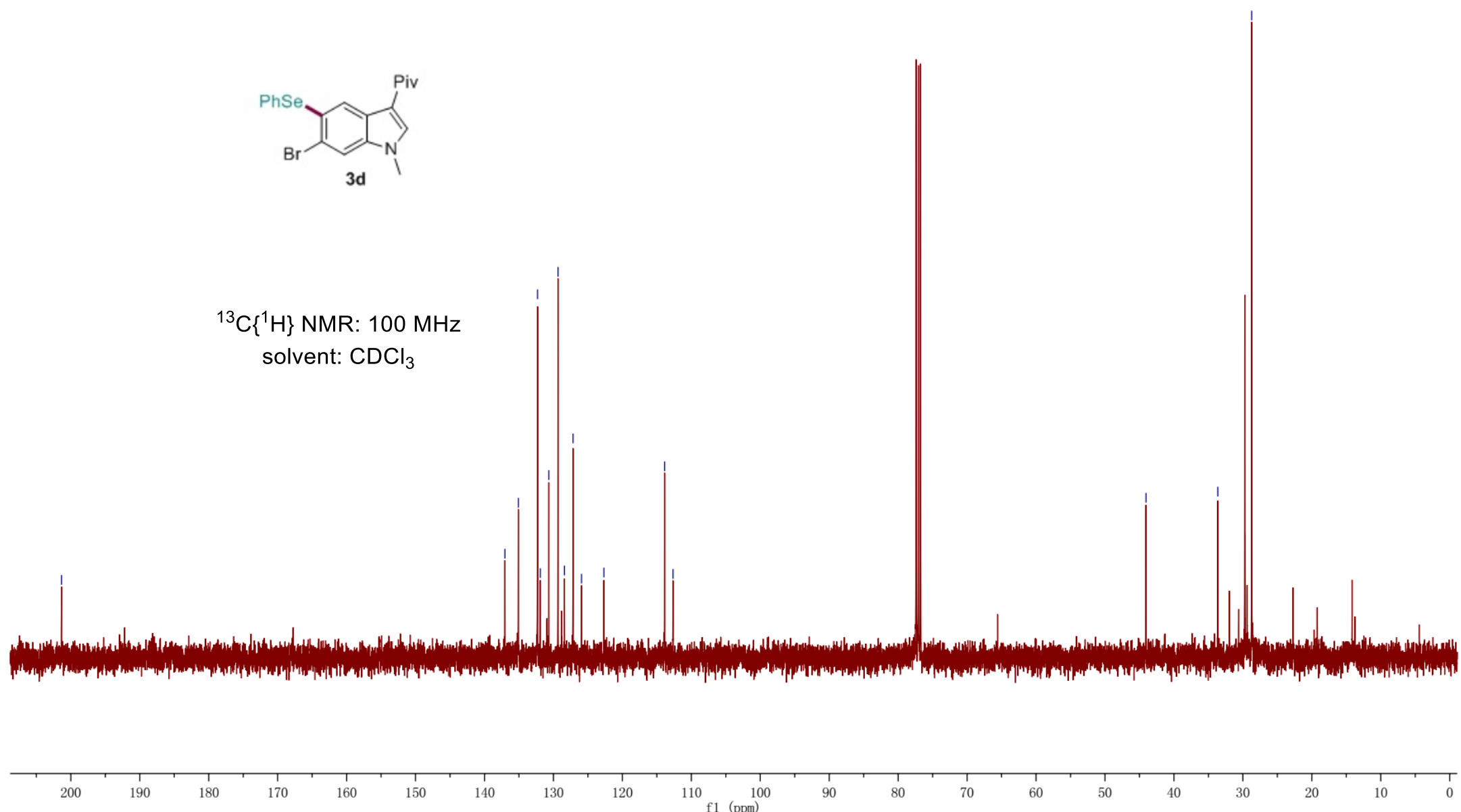

$110 \underset{\mathrm{f} 1}{\mathrm{ppm}} 100$ 


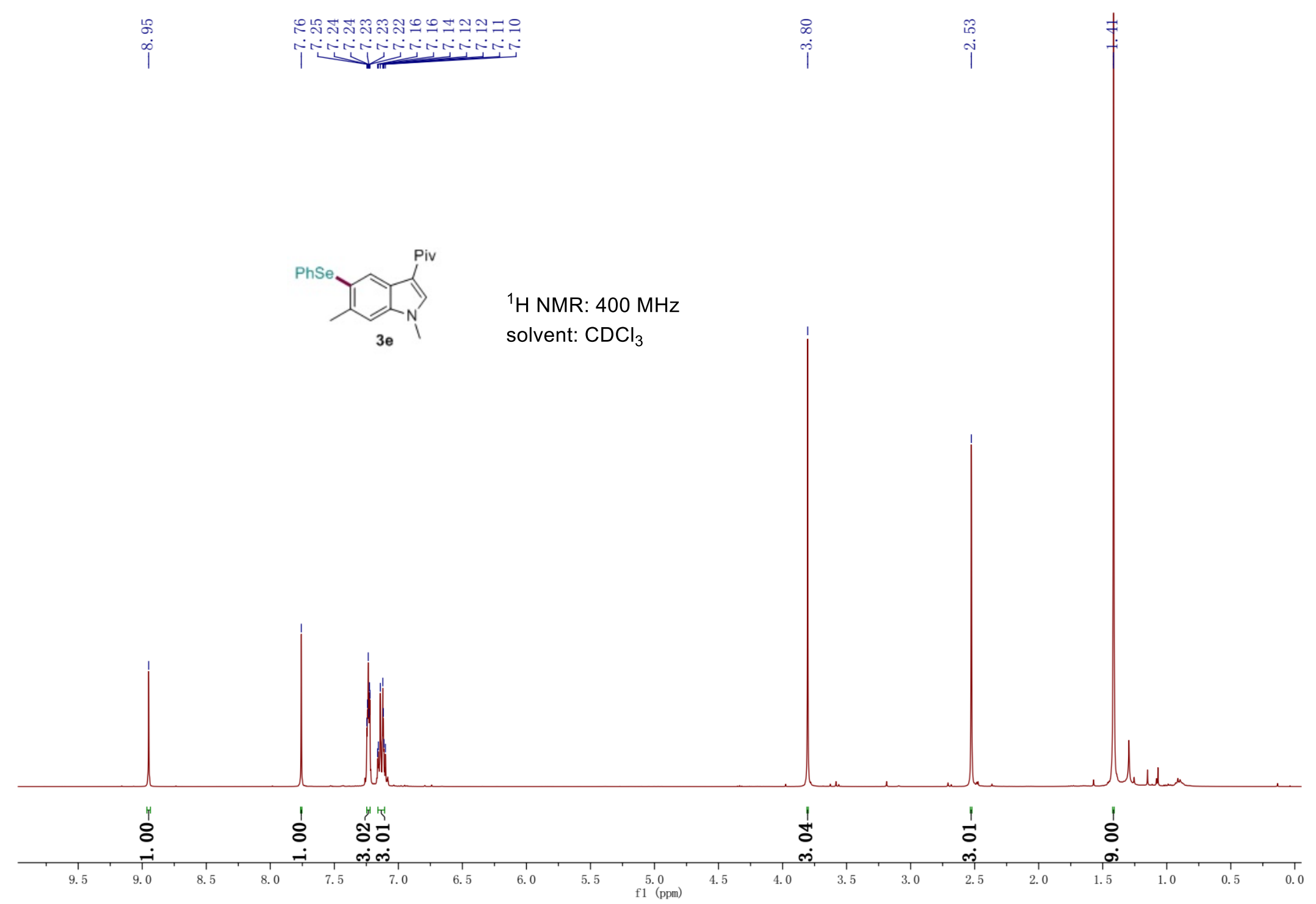



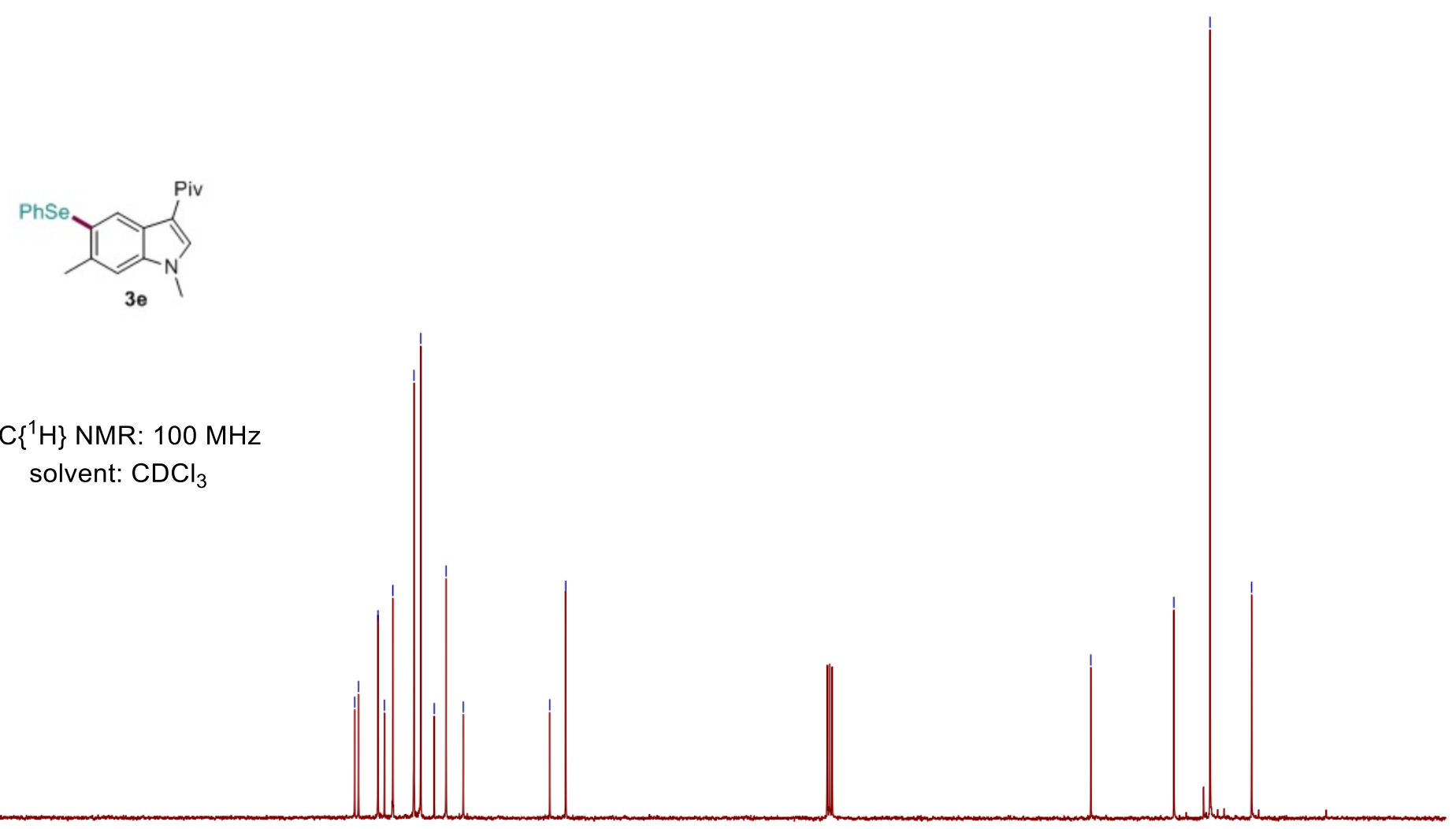

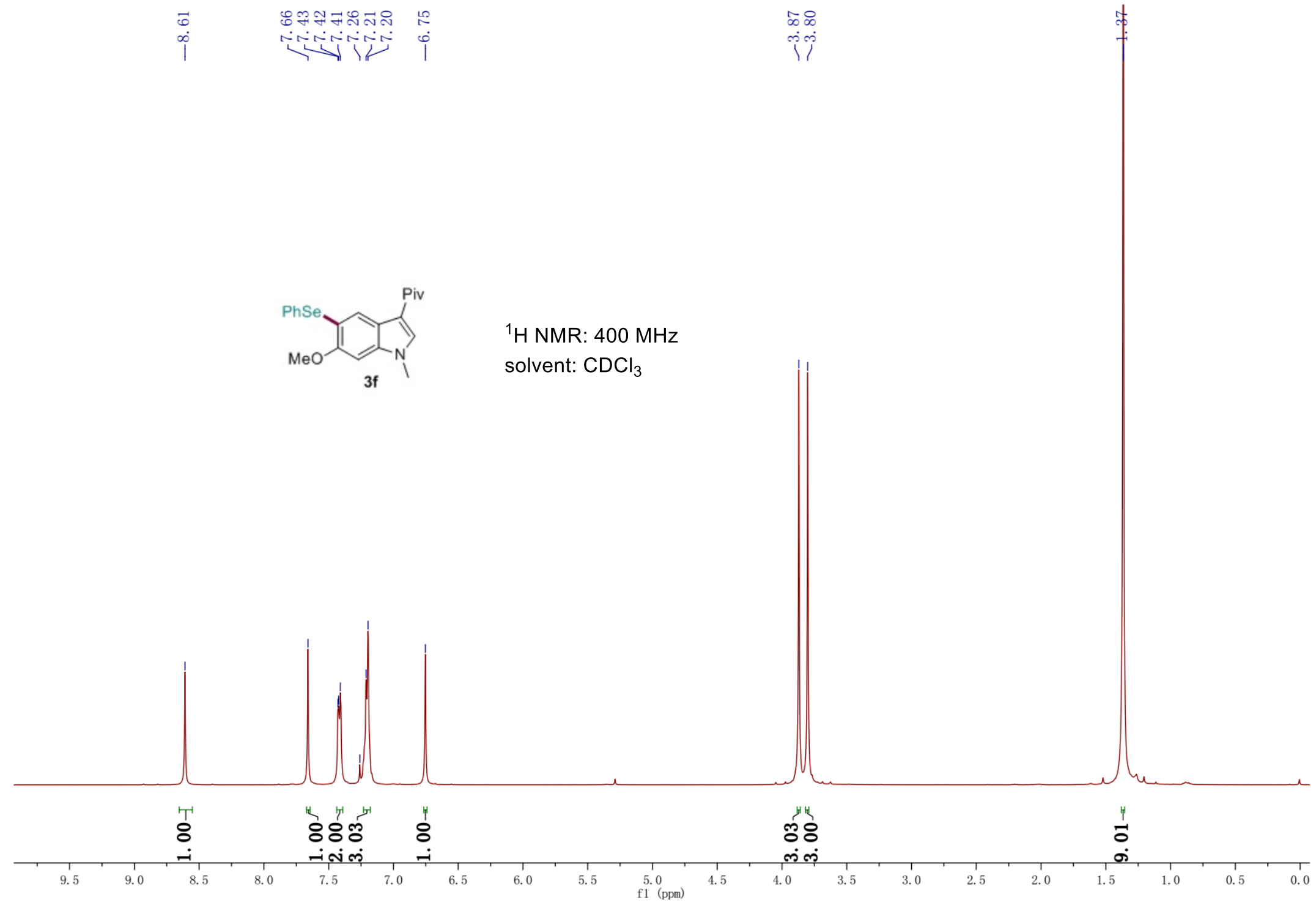


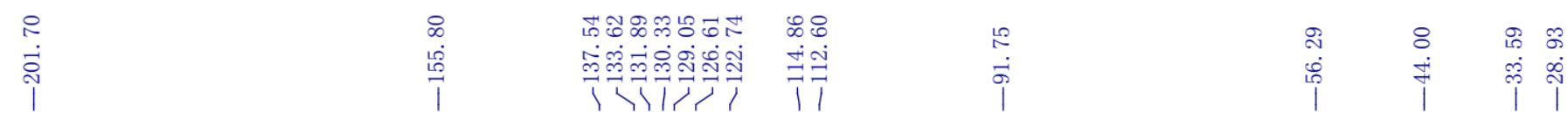
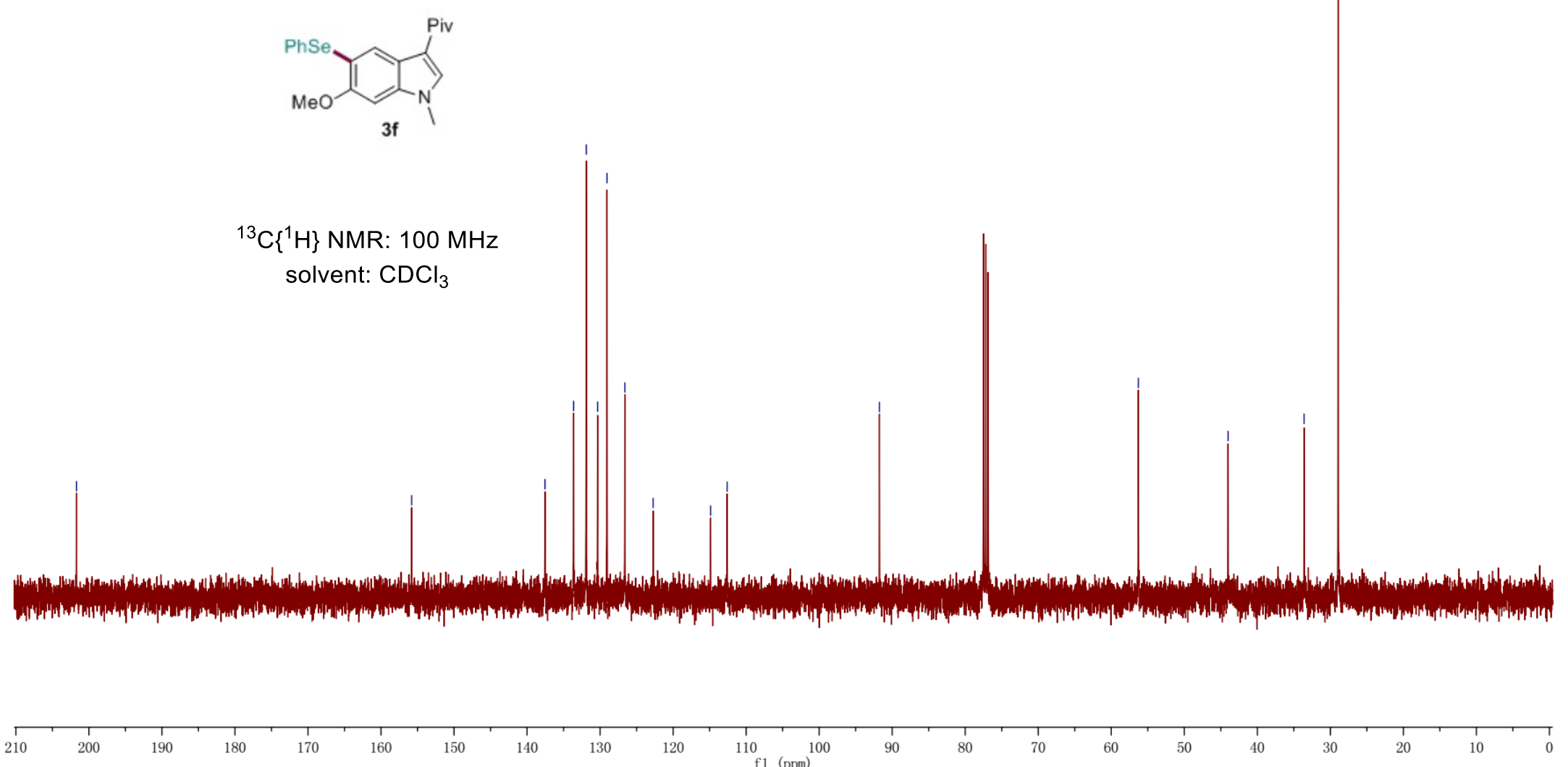


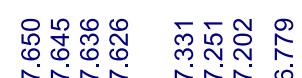

선 弥i

辛

$\stackrel{i}{\stackrel{2}{*}}$

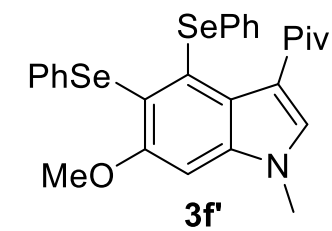

${ }^{1} \mathrm{H}$ NMR: $400 \mathrm{MHz}$

solvent: $\mathrm{CDCl}_{3}$

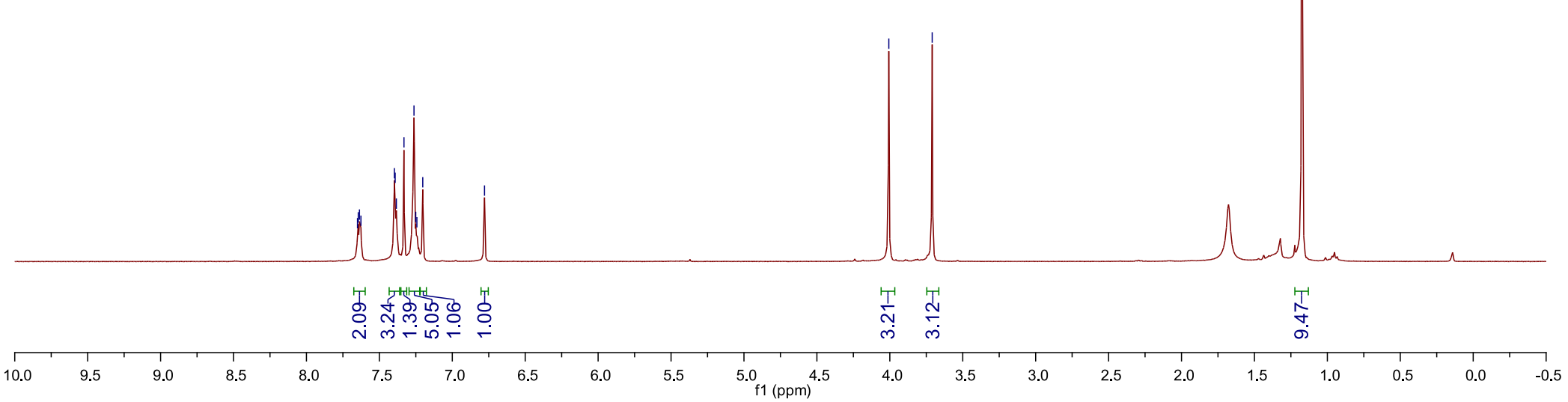




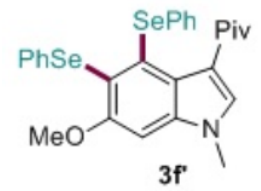

$1+$

${ }^{13} \mathrm{C}\left\{{ }^{1} \mathrm{H}\right\}$ NMR: $100 \mathrm{MHz}$ solvent: $\mathrm{CDCl}_{3}$
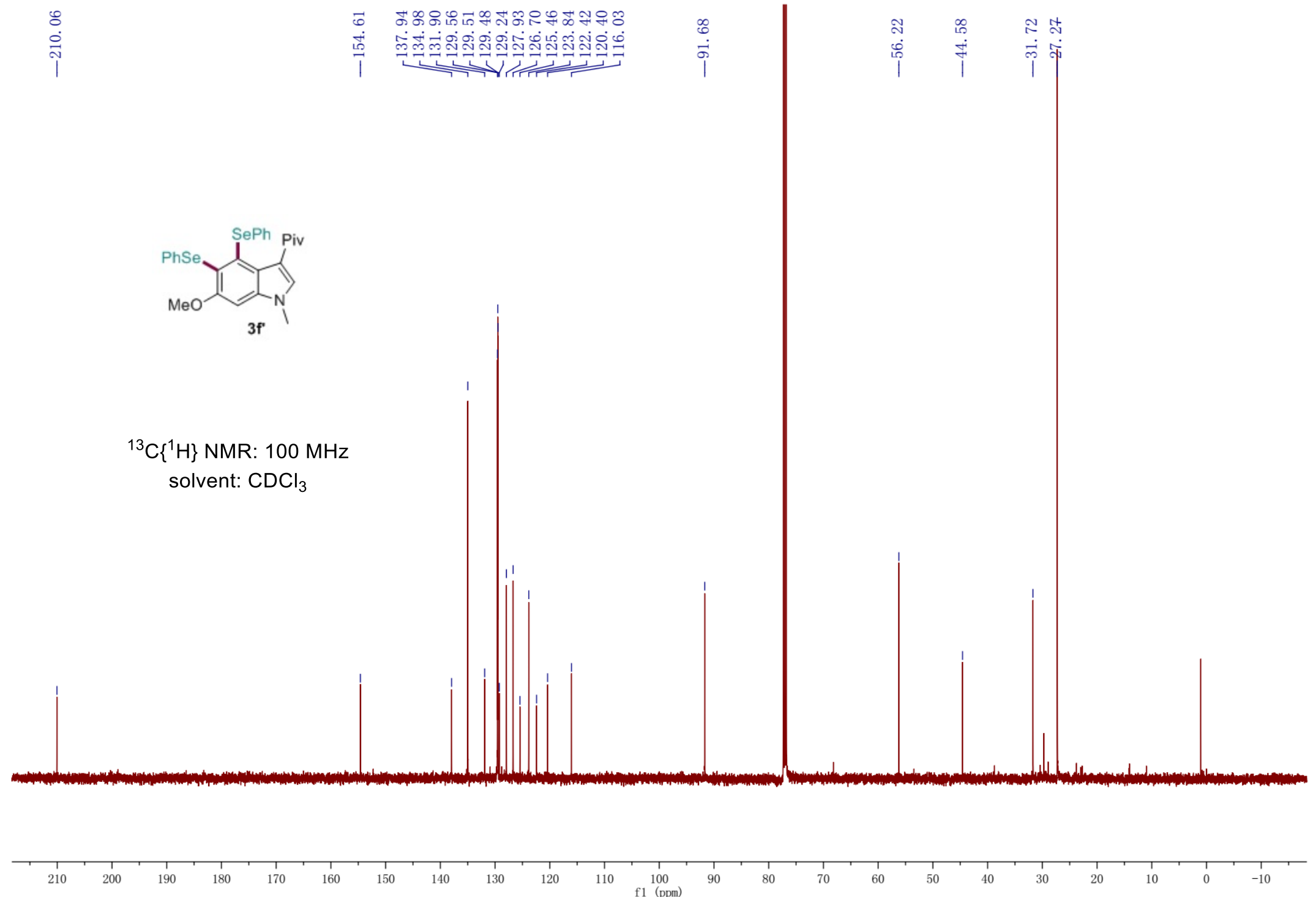


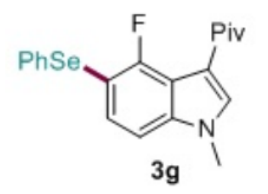

${ }^{1} \mathrm{H}$ NMR: $400 \mathrm{MHz}$

solvent: $\mathrm{CDCl}_{3}$

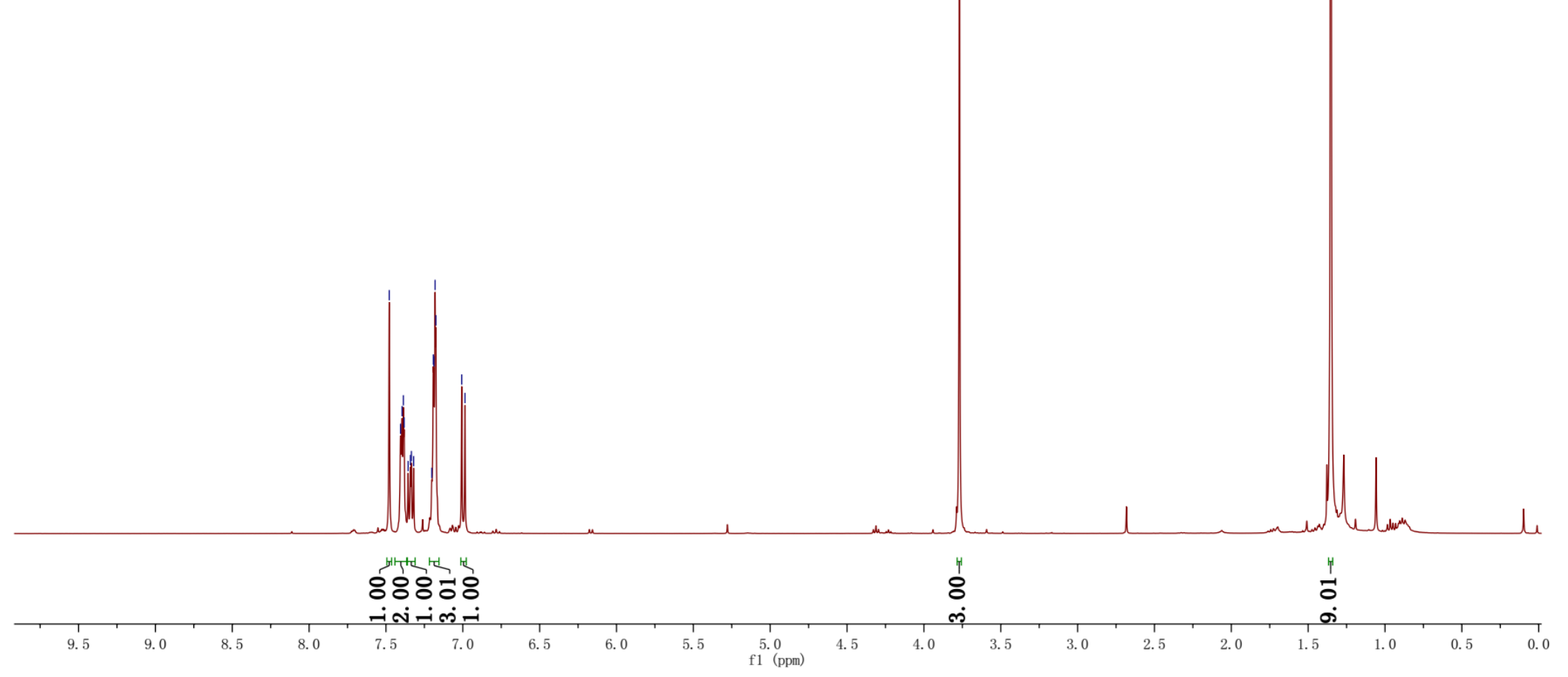




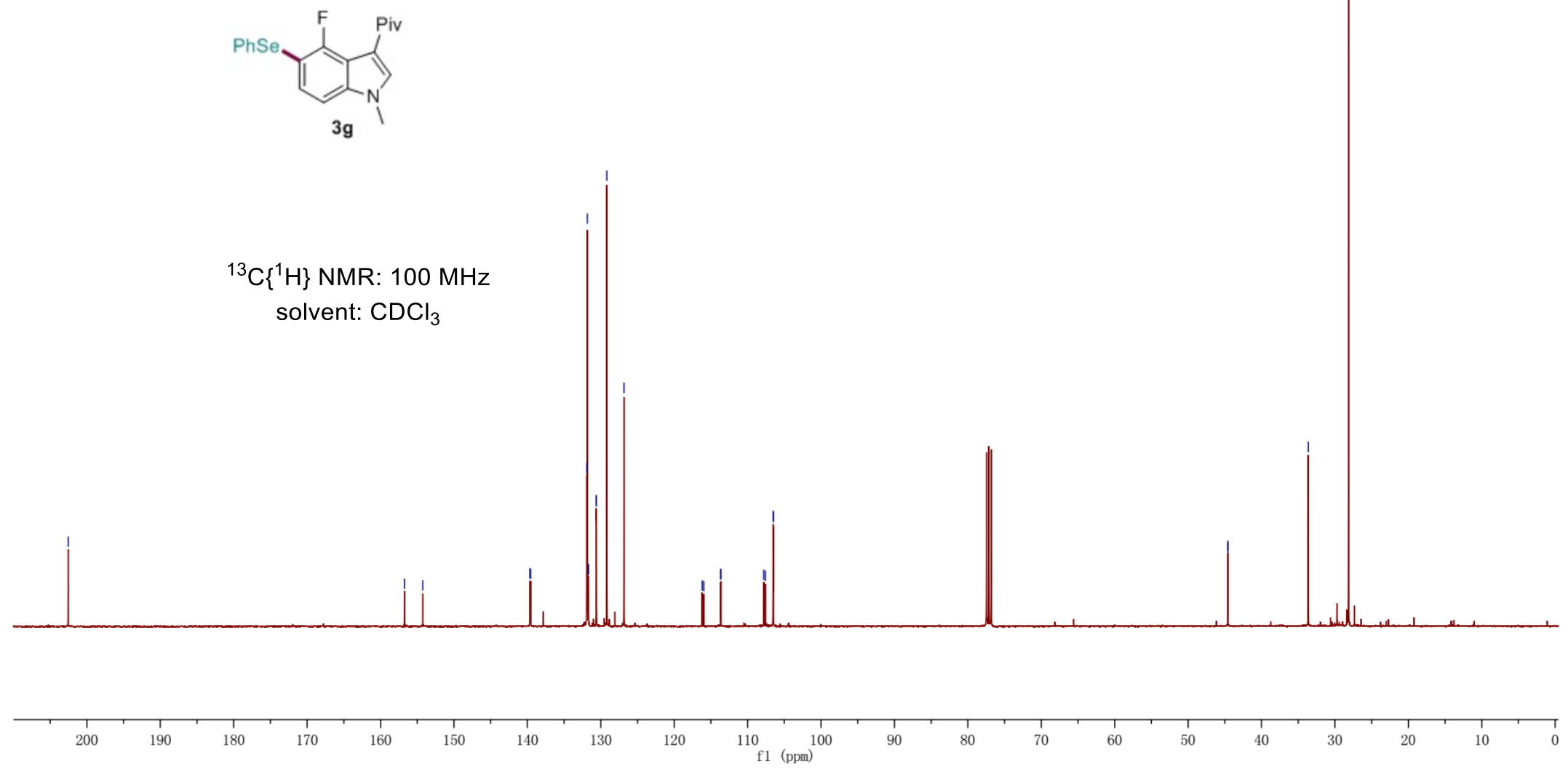




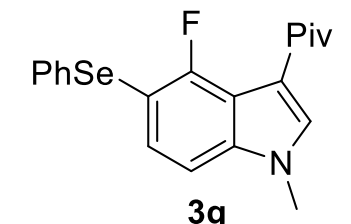

${ }^{19} \mathrm{~F}$ NMR: $377 \mathrm{MHz}$

Solvent: $\mathrm{CDCl}_{3}$ 
ชำ

NNNNNNNNNN

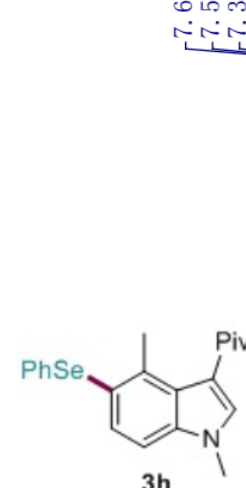

3h
${ }^{1} \mathrm{H}$ NMR: $400 \mathrm{MHz}$

solvent: $\mathrm{CDCl}_{3}$

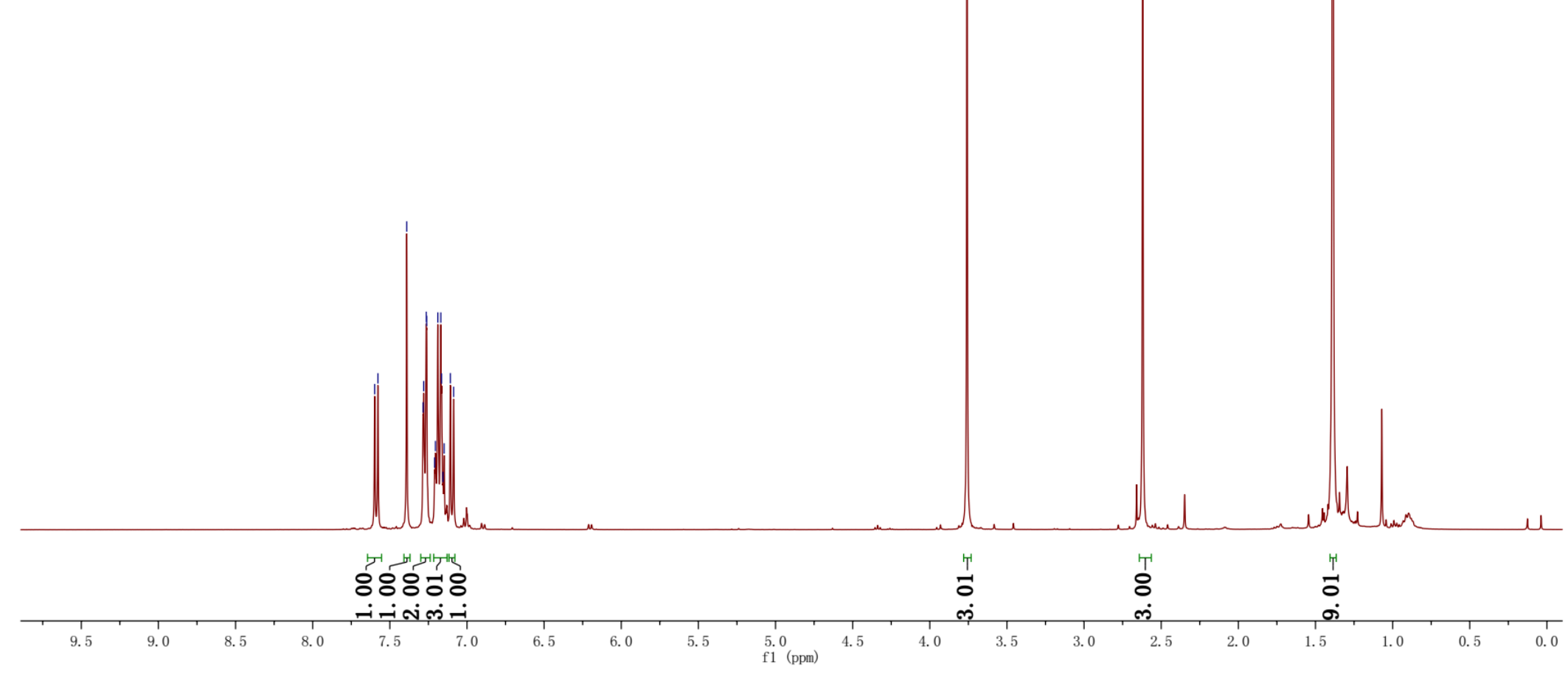




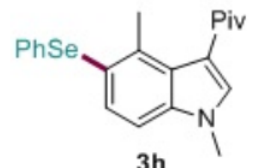

${ }^{13} \mathrm{C}\left\{{ }^{1} \mathrm{H}\right\}$ NMR: $100 \mathrm{MHz}$ solvent: $\mathrm{CDCl}_{3}$
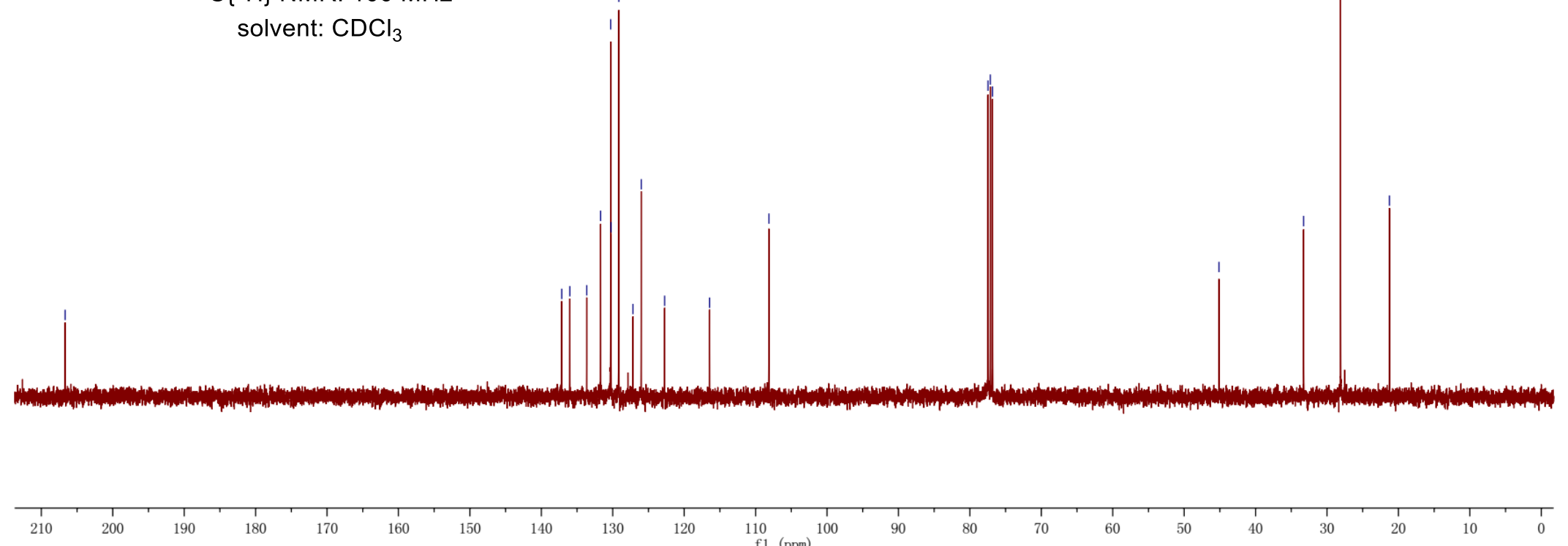


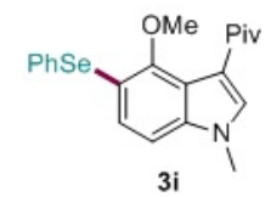

${ }^{1} \mathrm{H}$ NMR: $400 \mathrm{MHz}$

solvent: $\mathrm{CDCl}_{3}$

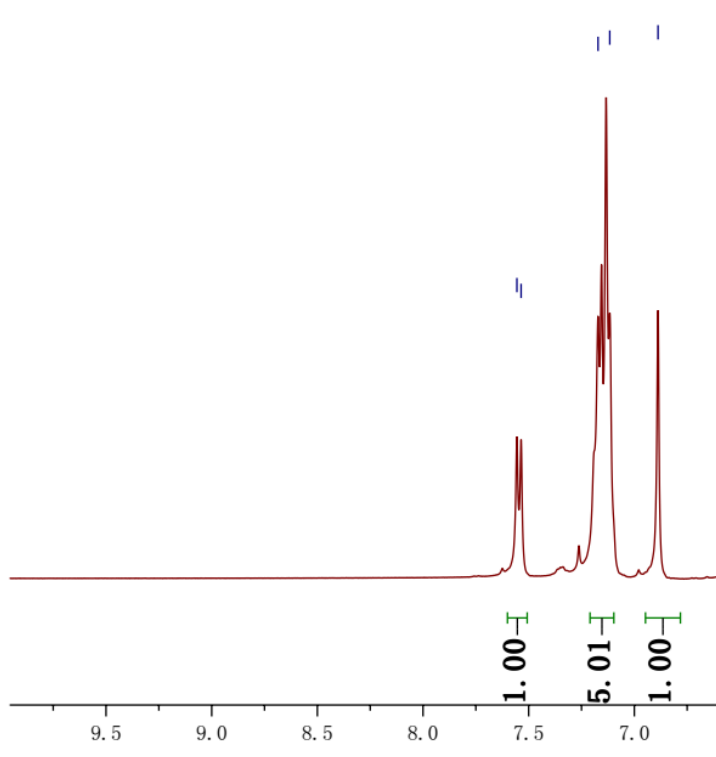




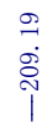

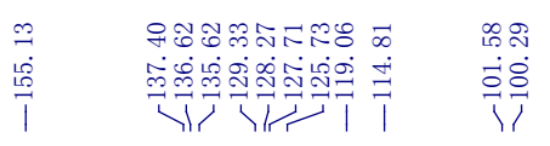
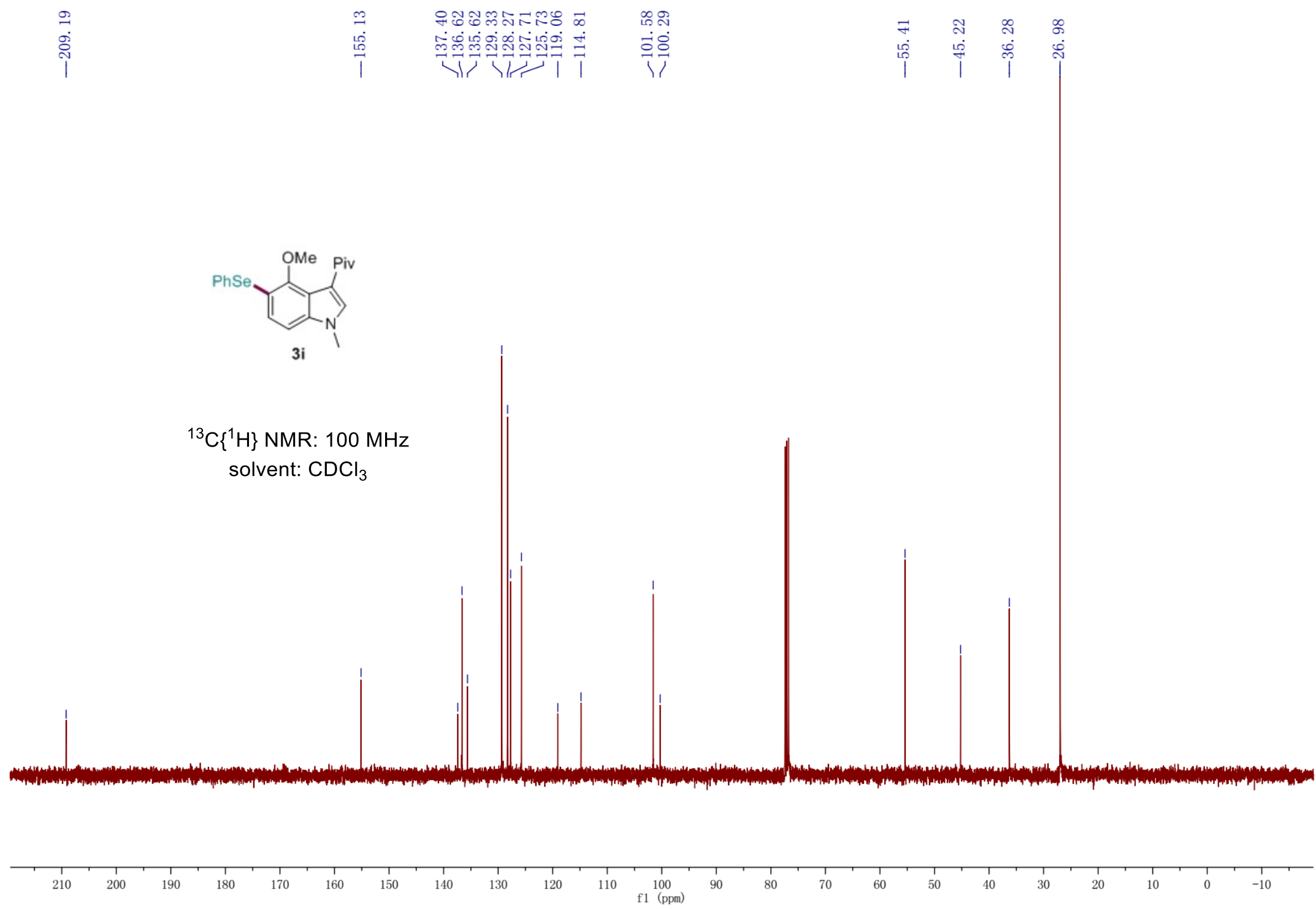

S47 


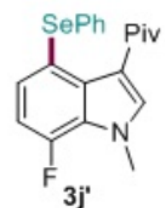

${ }^{1} \mathrm{H}$ NMR: $400 \mathrm{MHz}$

solvent: $\mathrm{CDCl}_{3}$

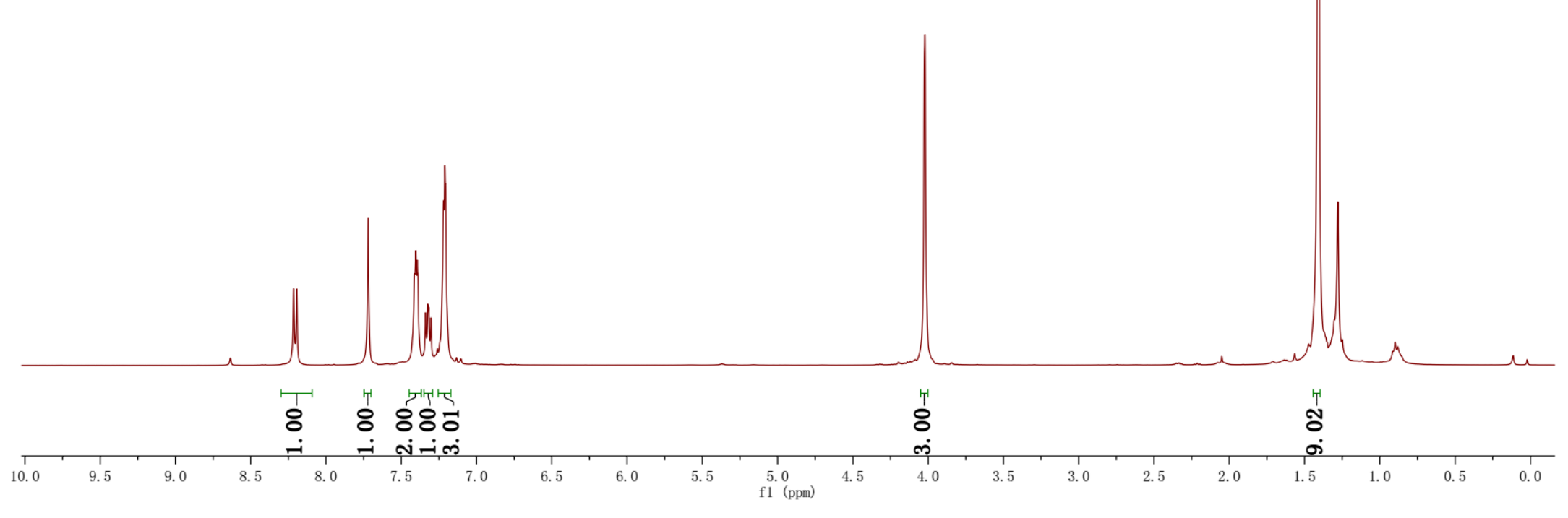




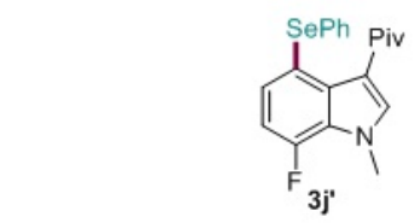

${ }^{13} \mathrm{C}\left\{{ }^{1} \mathrm{H}\right\}$ NMR: $100 \mathrm{MHz}$ solvent: $\mathrm{CDCl}_{3}$

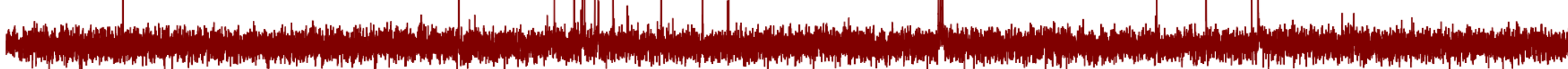

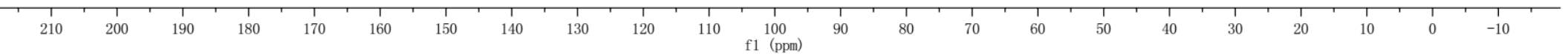




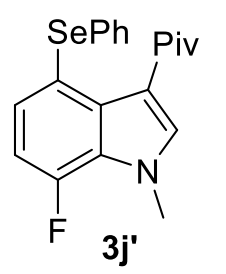

${ }^{19}$ F NMR: $377 \mathrm{MHz}$

Solvent: $\mathrm{CDCl}_{3}$ 


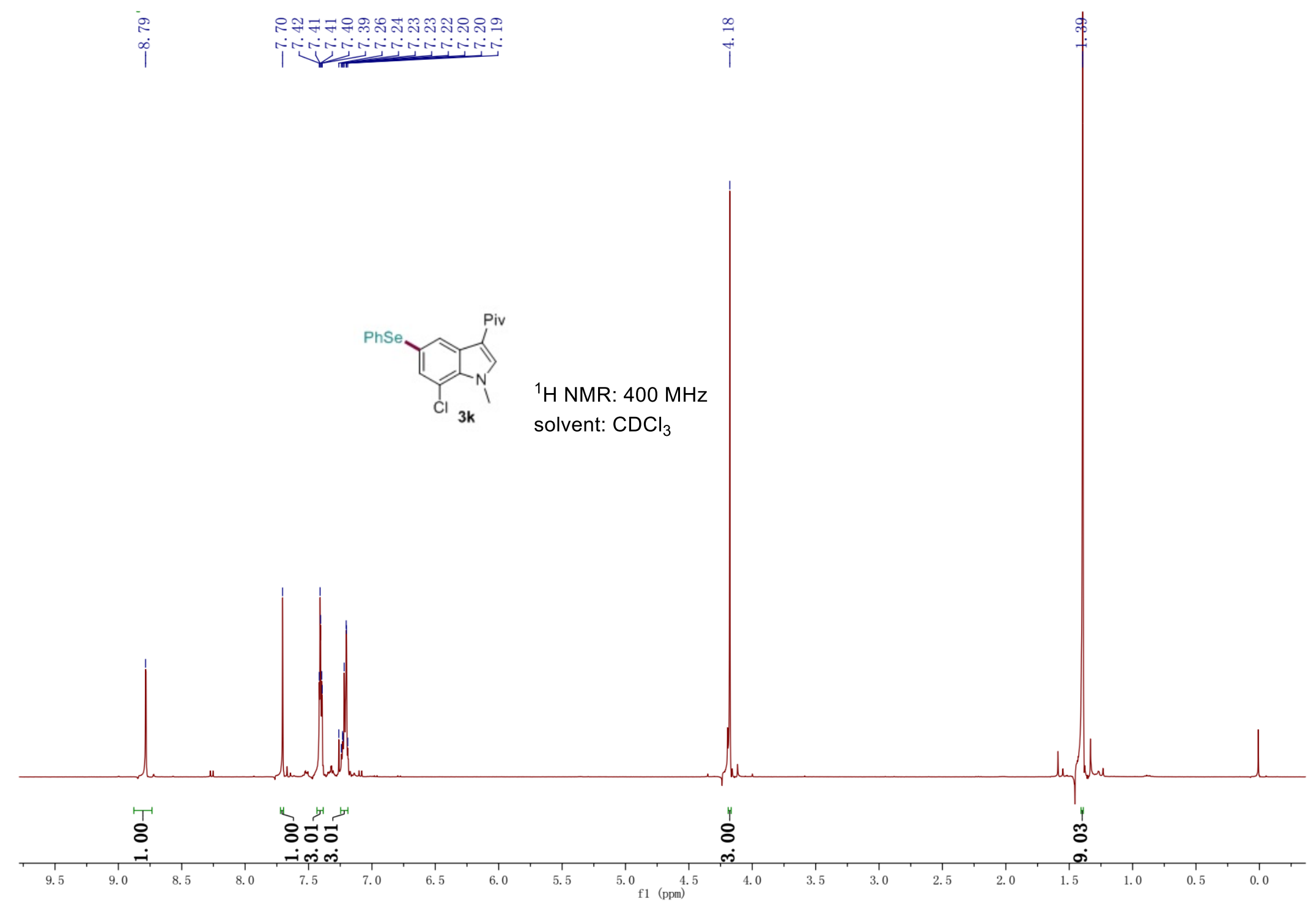


${ }^{13} \mathrm{C}\left\{{ }^{1} \mathrm{H}\right\}$ NMR: $100 \mathrm{MHz}$ solvent: $\mathrm{CDCl}_{3}$
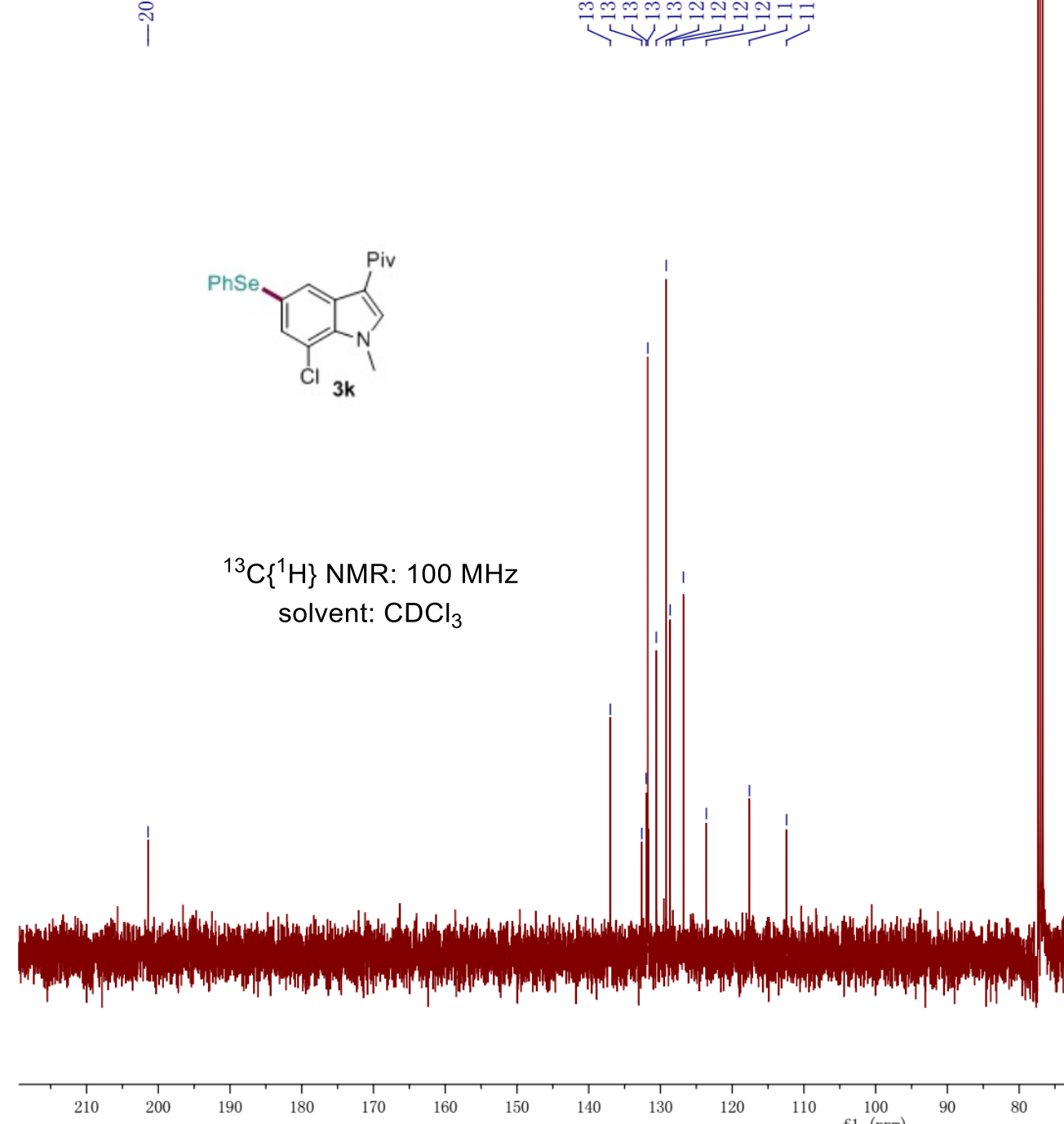

$110 \quad \begin{gathered}100 \\ \mathrm{f} 1(\mathrm{ppm})\end{gathered}$ 1
$90 \quad 80$ 


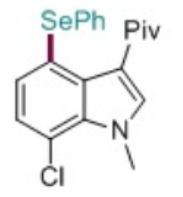

${ }^{1} \mathrm{H}$ NMR: $400 \mathrm{MHz}$

solvent: $\mathrm{CDCl}_{3}$

$3 k^{\prime}$

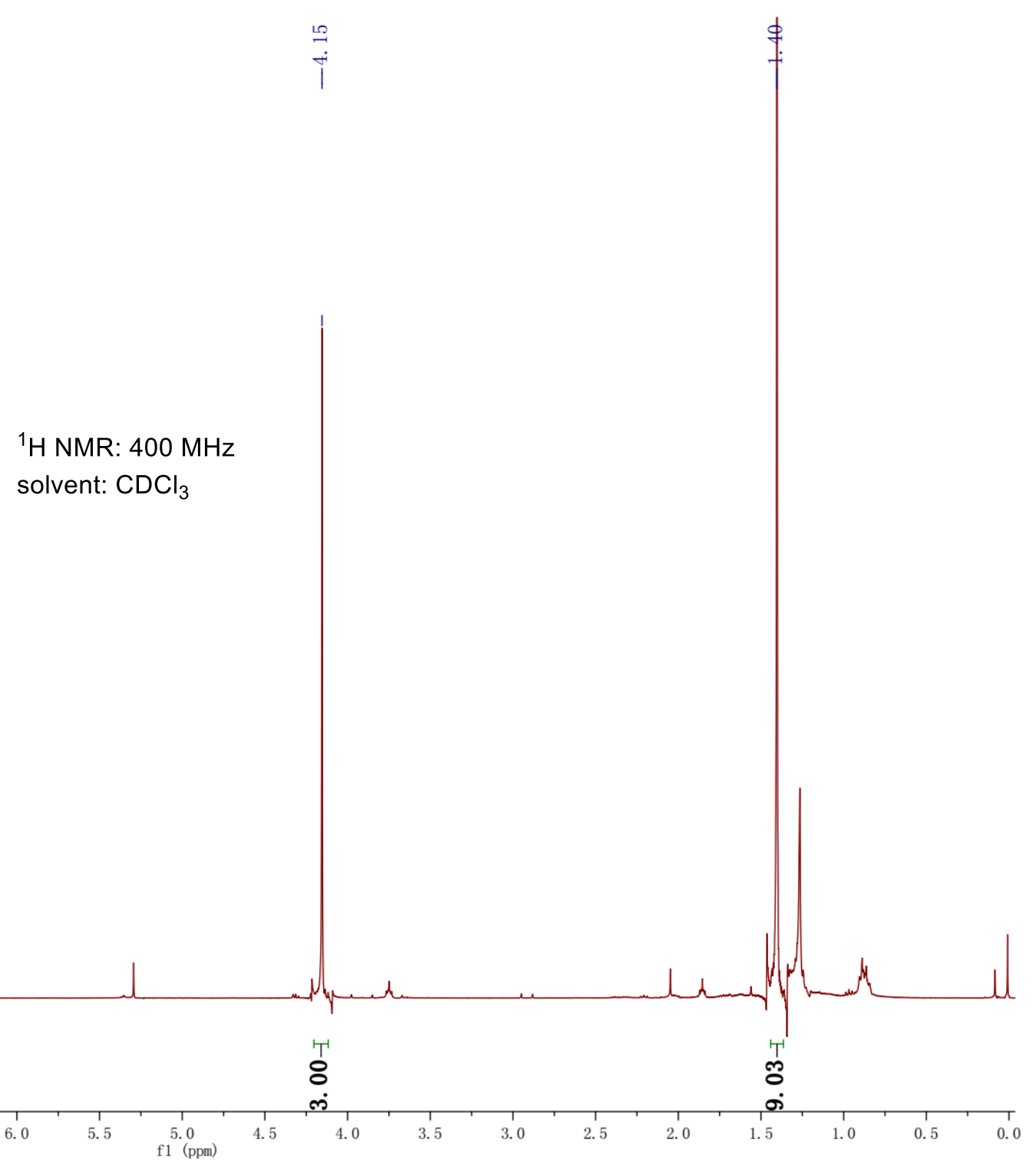




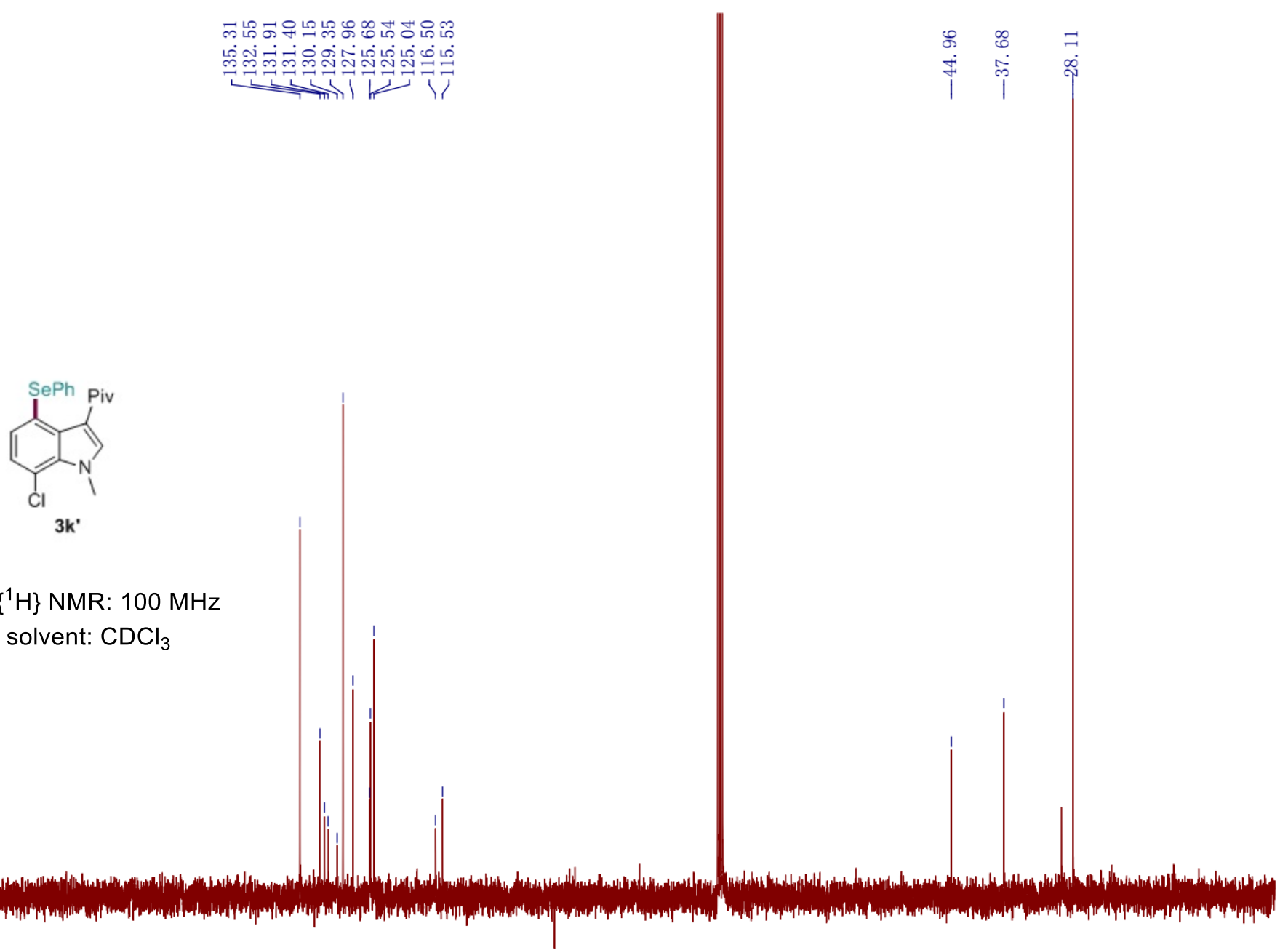

$\frac{1}{210}$

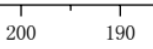

180 170 $160 \quad 150$

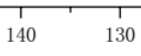
120 $\begin{array}{ll}110 & 100 \\ \mathrm{f} 1(\mathrm{ppm}) & \end{array}$ 90 1
$80 \quad 70$ 60 1
$50 \quad 40$ $30 \quad 2$ 10 


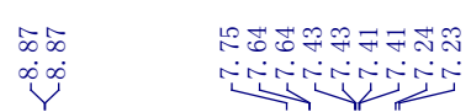

i

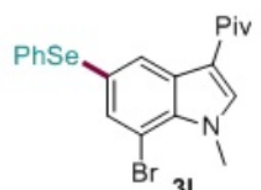

31

${ }^{1} \mathrm{H}$ NMR: $400 \mathrm{MHz}$

solvent: $\mathrm{CDCl}_{3}$

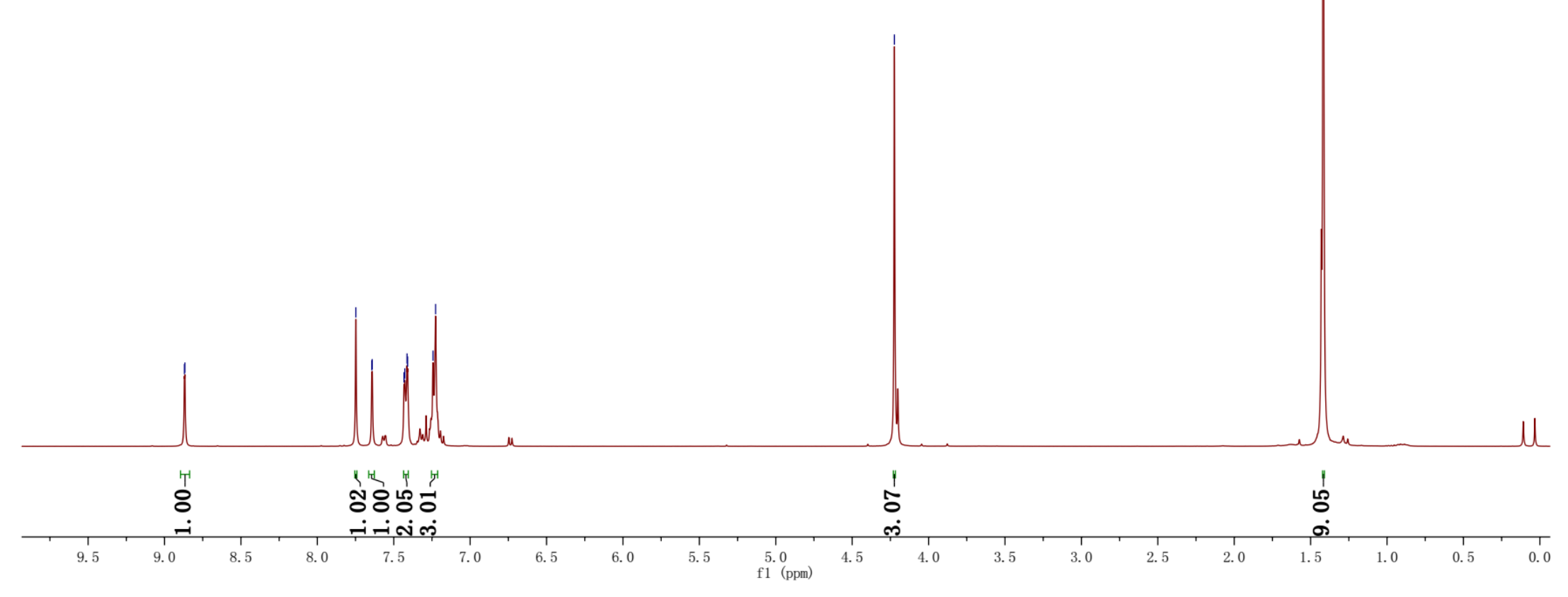




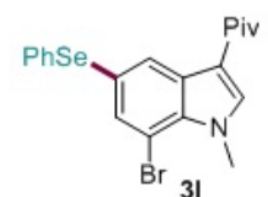

${ }^{13} \mathrm{C}\left\{{ }^{1} \mathrm{H}\right\}$ NMR: $100 \mathrm{MHz}$ solvent: $\mathrm{CDCl}_{3}$
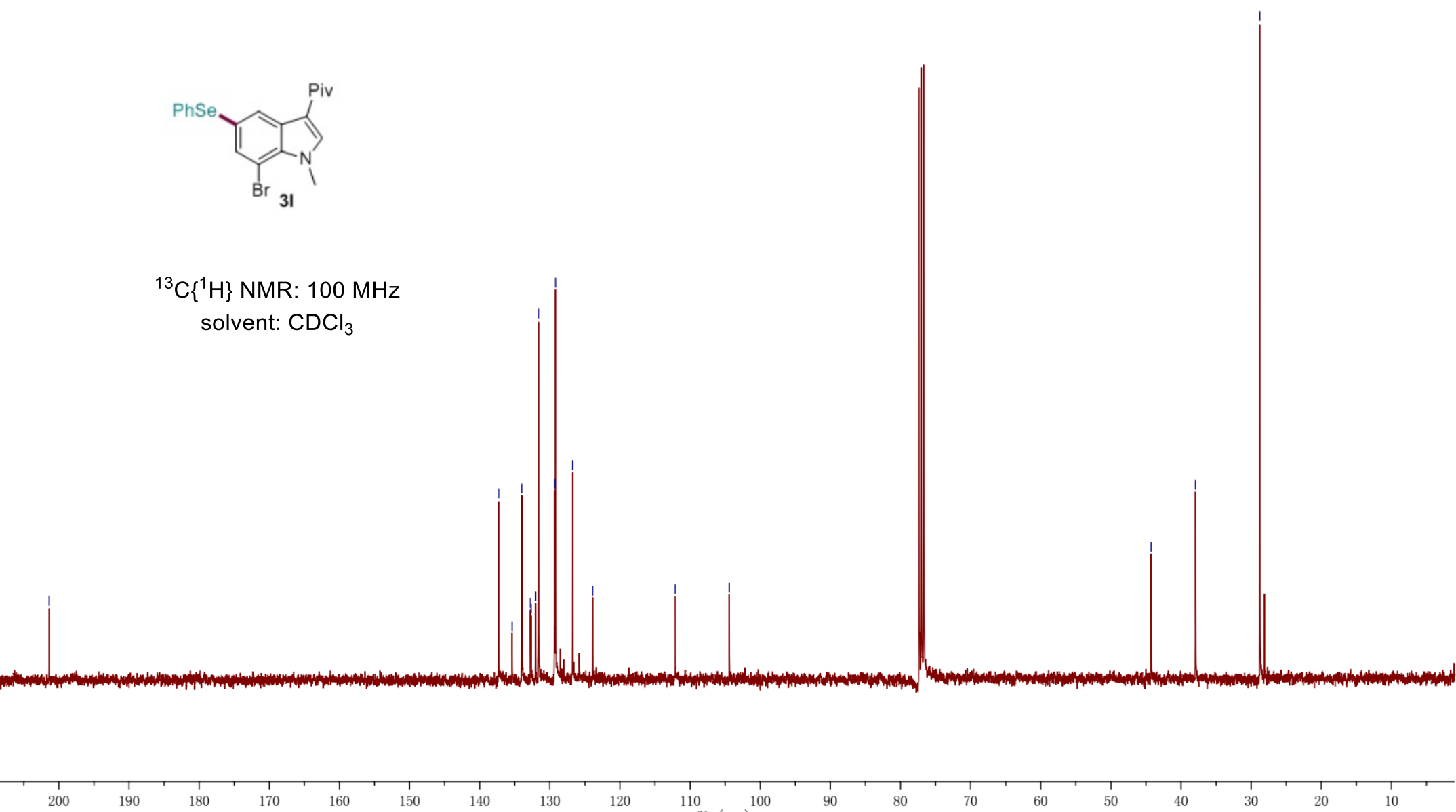


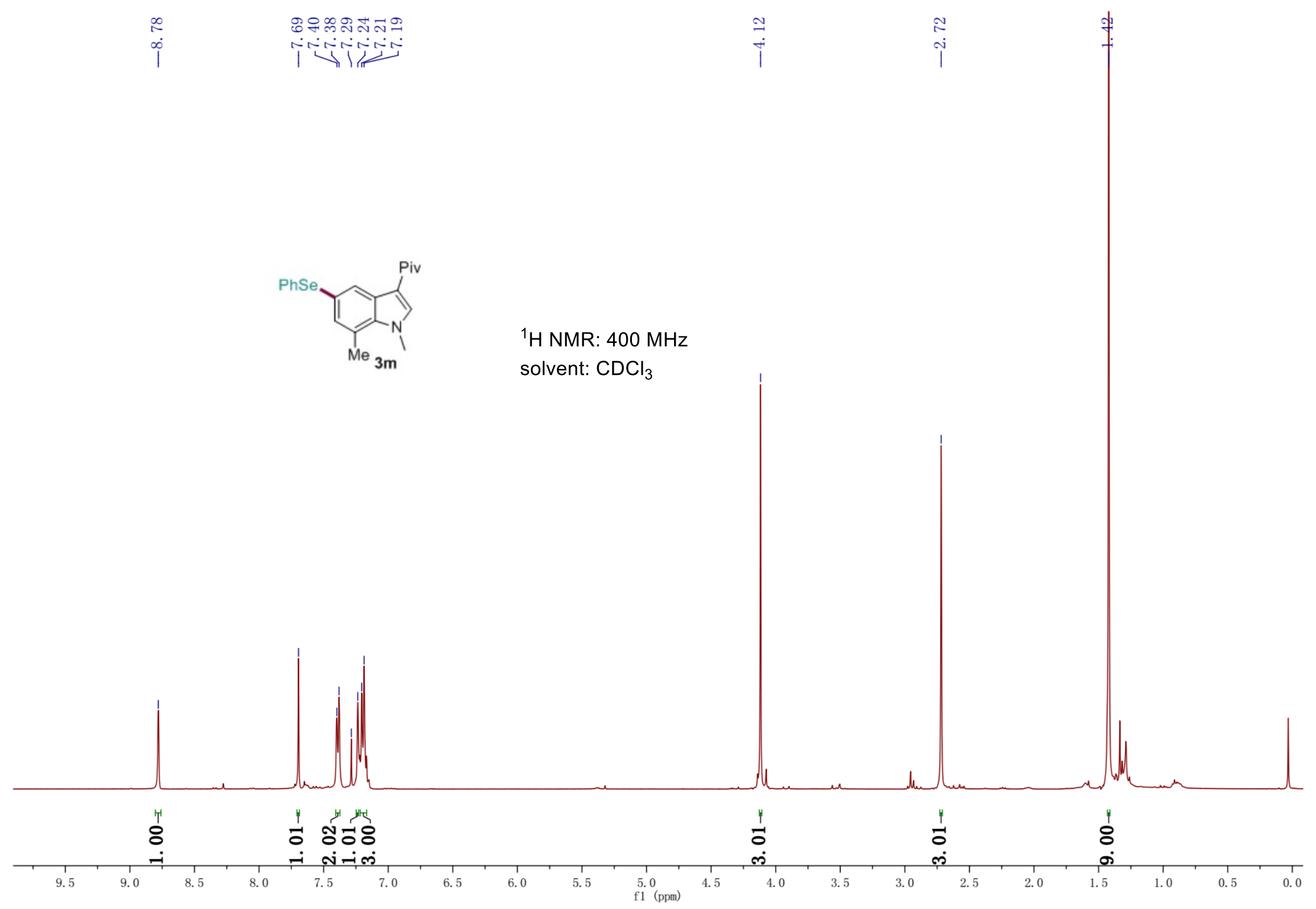




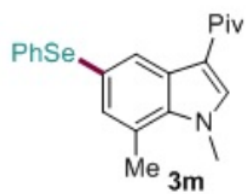

${ }^{13} \mathrm{C}\left\{{ }^{1} \mathrm{H}\right\}$ NMR: $100 \mathrm{MHz}$ solvent: $\mathrm{CDCl}_{3}$

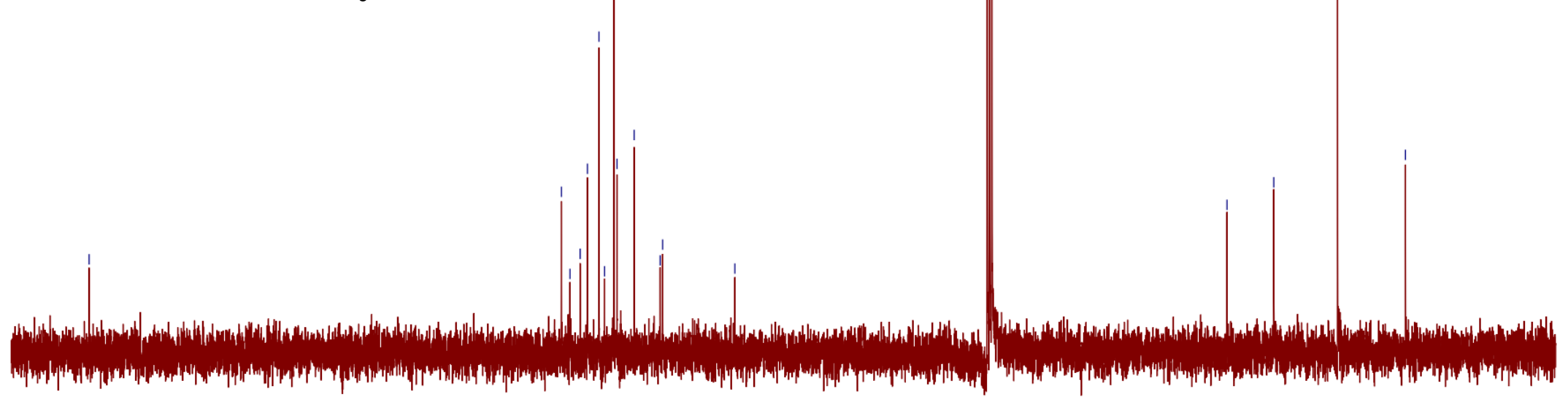
$200 \quad 190$ 180 170 1
$160 \quad 150$ 140 130
130 ${ }_{\mathrm{f} 1(\mathrm{ppm})}^{100}$ 


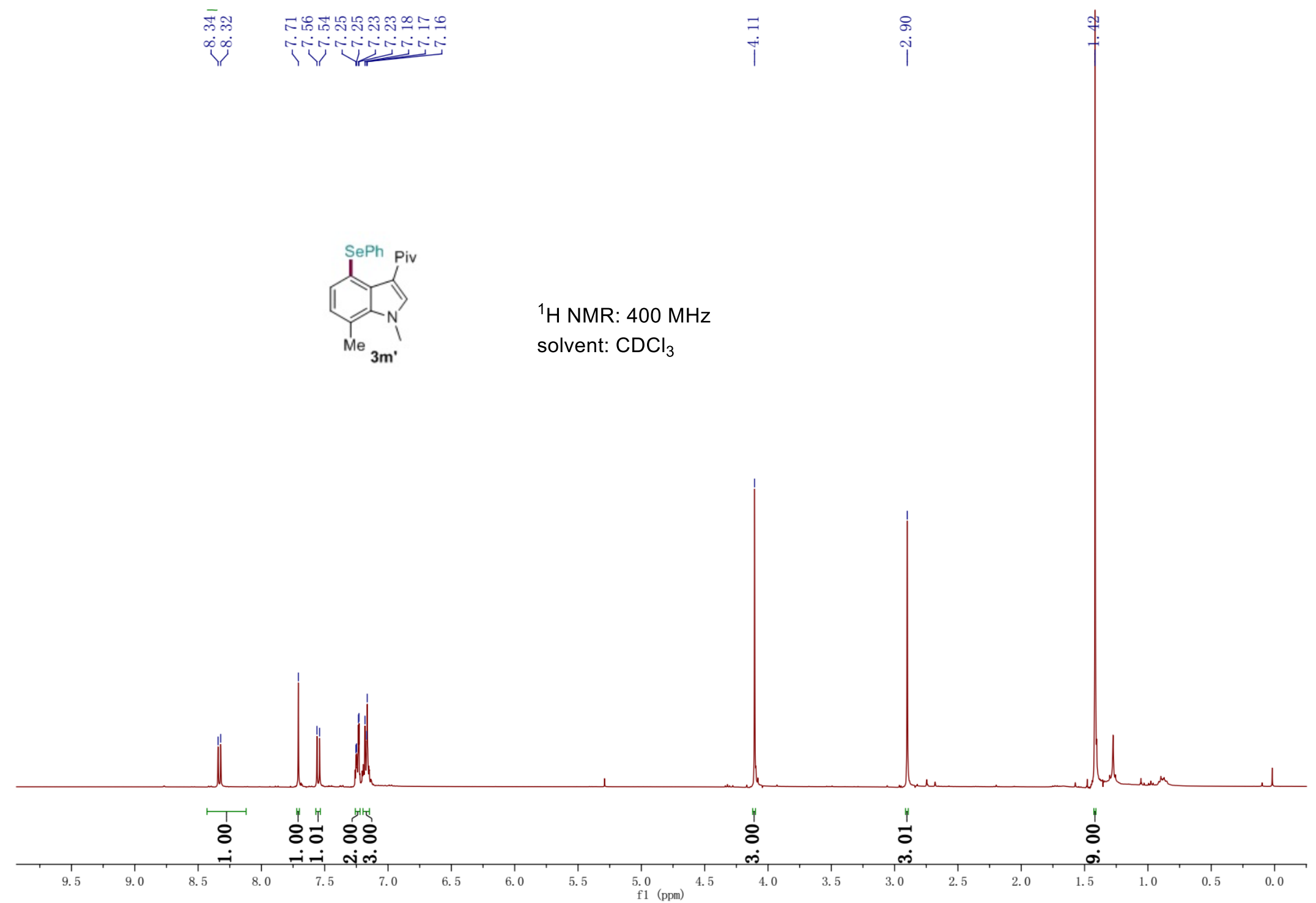




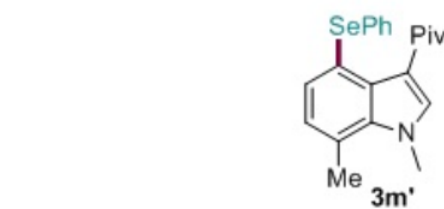

$3 \mathrm{~m}^{\prime}$

${ }^{13} \mathrm{C}\left\{{ }^{1} \mathrm{H}\right\}$ NMR: $100 \mathrm{MHz}$ solvent: $\mathrm{CDCl}_{3}$

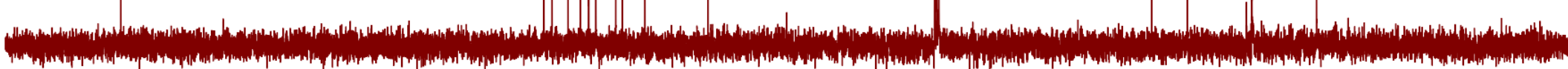

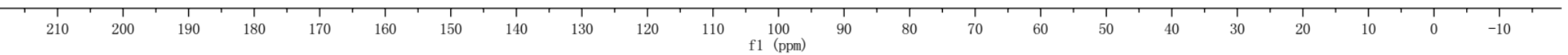




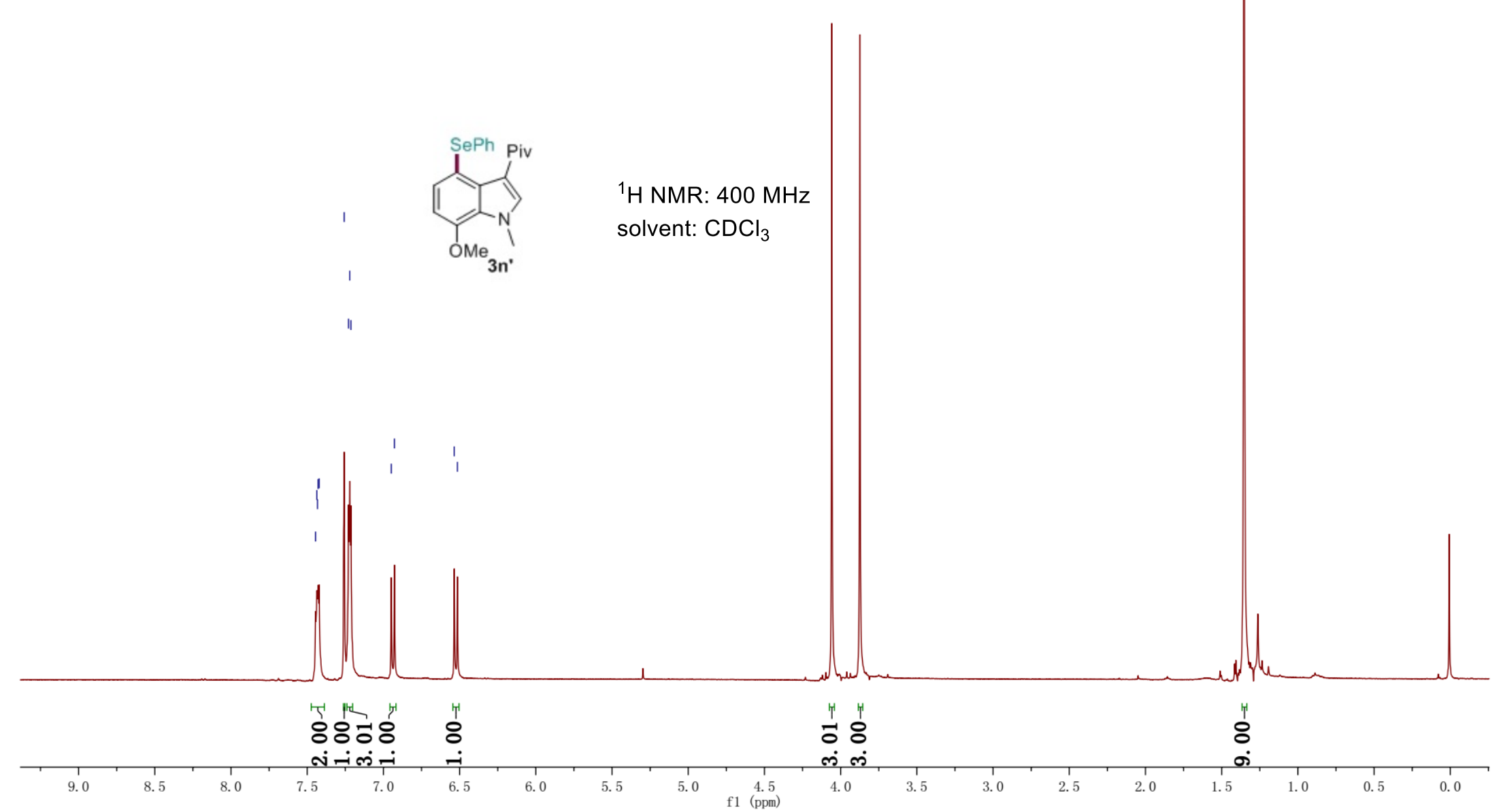




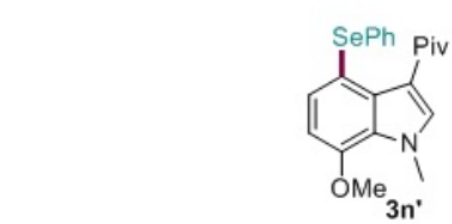

${ }^{13} \mathrm{C}\left\{{ }^{1} \mathrm{H}\right\}$ NMR: $100 \mathrm{MHz}$ solvent: $\mathrm{CDCl}_{3}$
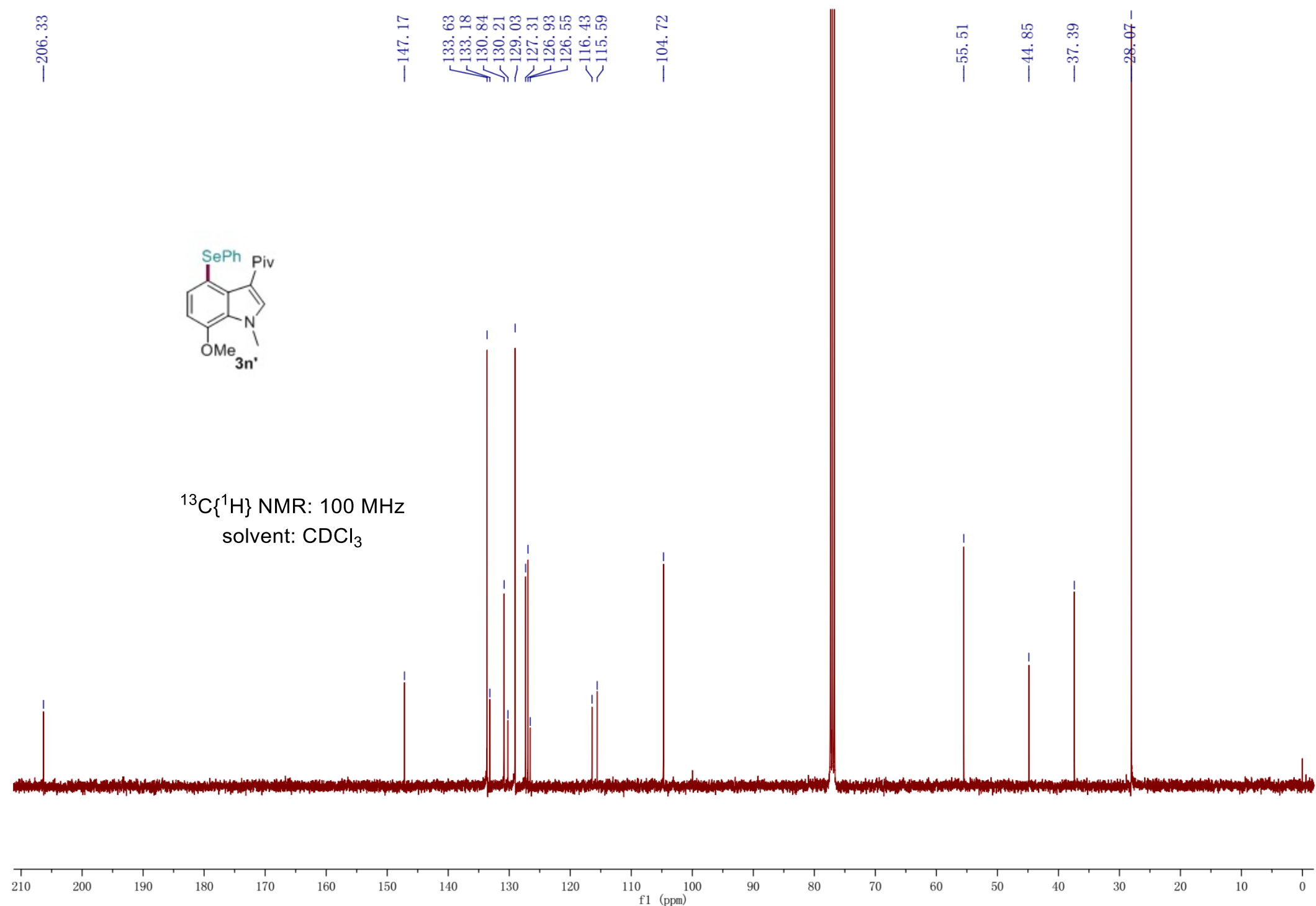


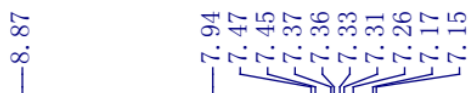

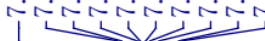

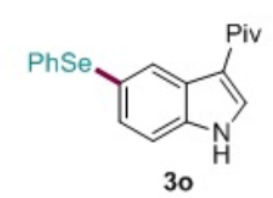

${ }^{1} \mathrm{H}$ NMR: $400 \mathrm{MHz}$

solvent: $\mathrm{CDCl}_{3}$

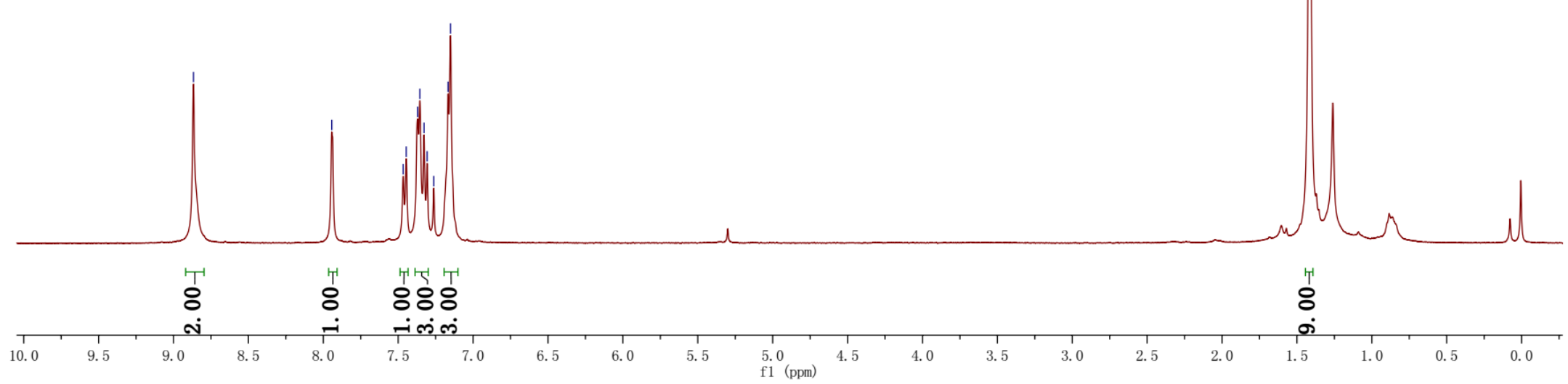




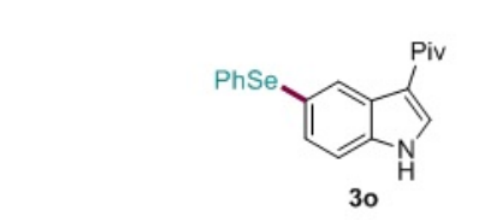

${ }^{13} \mathrm{C}\left\{{ }^{1} \mathrm{H}\right\}$ NMR: $100 \mathrm{MHz}$ solvent: $\mathrm{CDCl}_{3}$
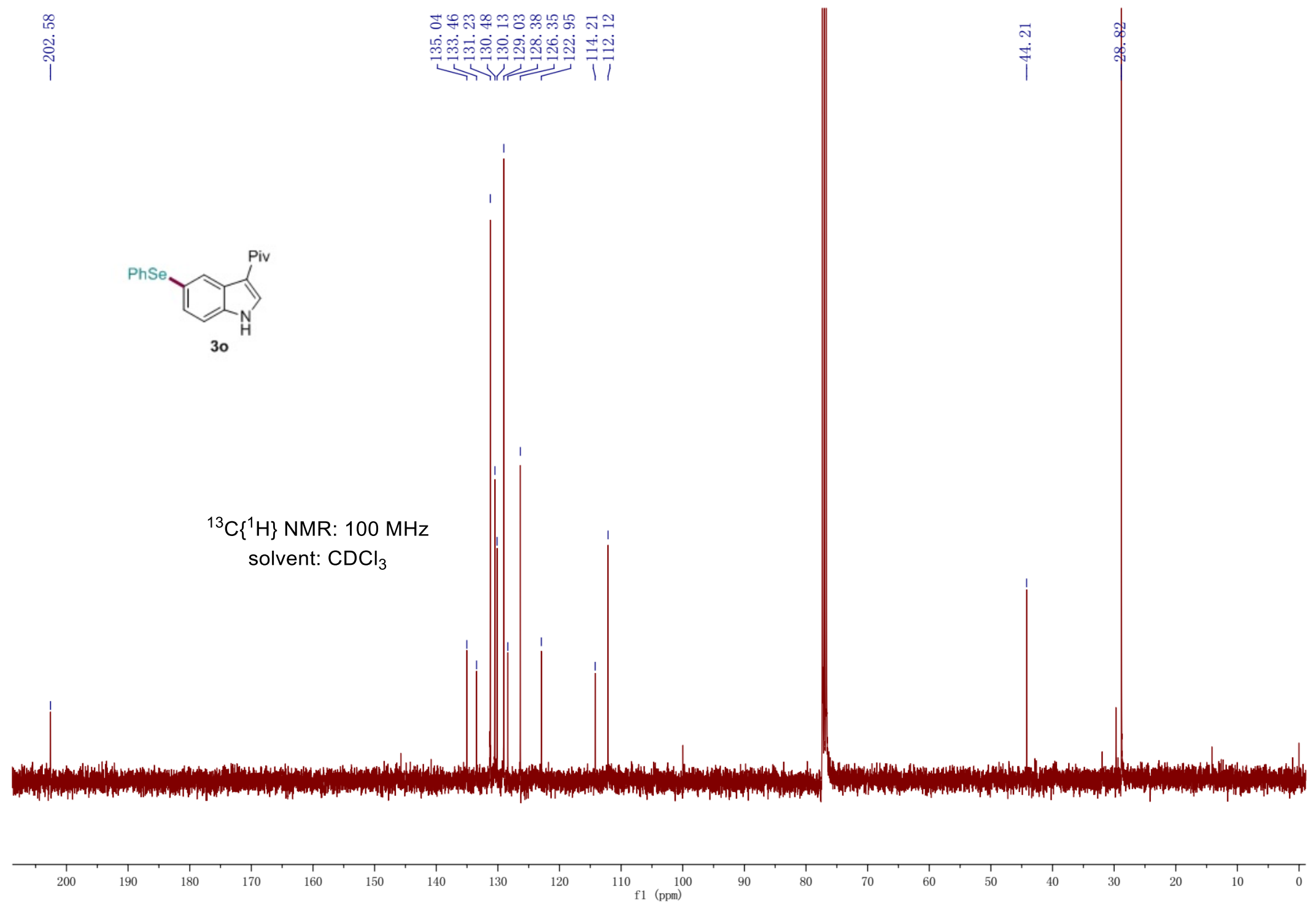


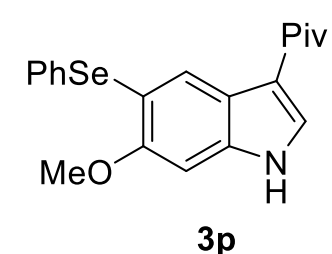

$3 p$
${ }^{1} \mathrm{H}$ NMR: $400 \mathrm{MHz}$

solvent: $\mathrm{CDCl}_{3}$ 


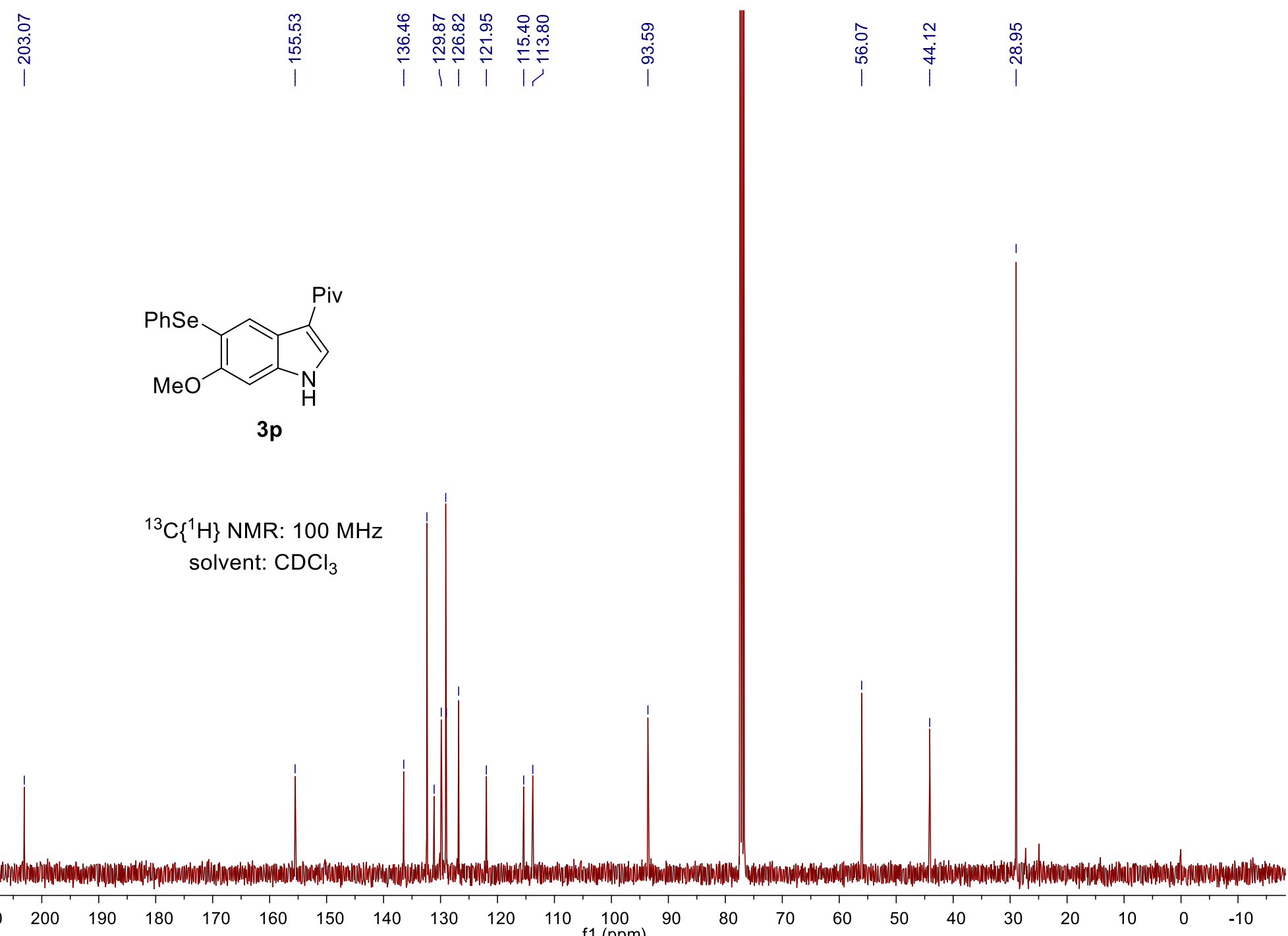




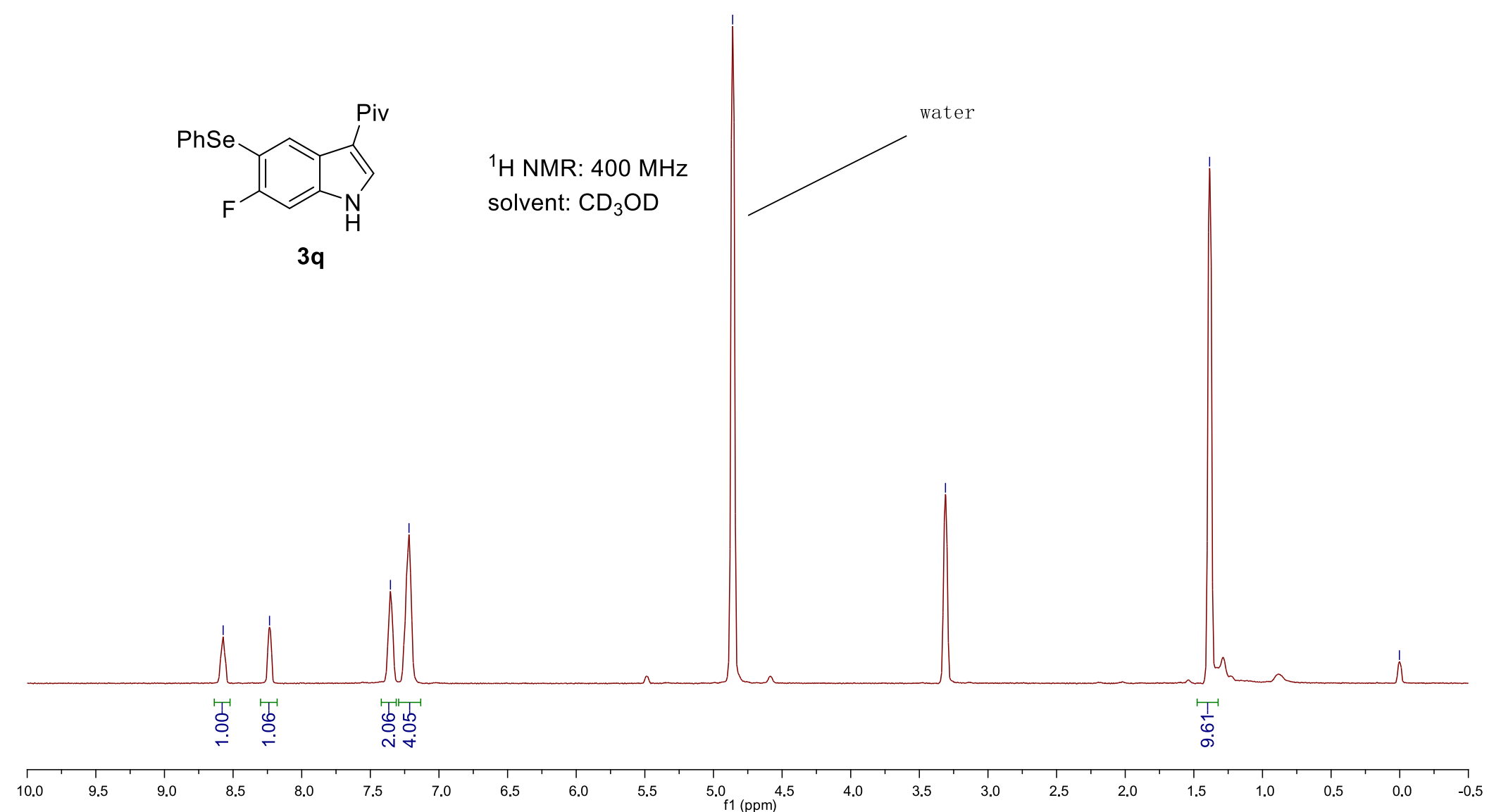




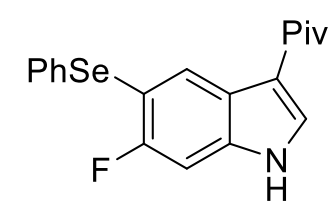

$3 q$

${ }^{13} \mathrm{C}\left\{{ }^{1} \mathrm{H}\right\}$ NMR: $100 \mathrm{MHz}$ solvent: $\mathrm{CD}_{3} \mathrm{OD}$
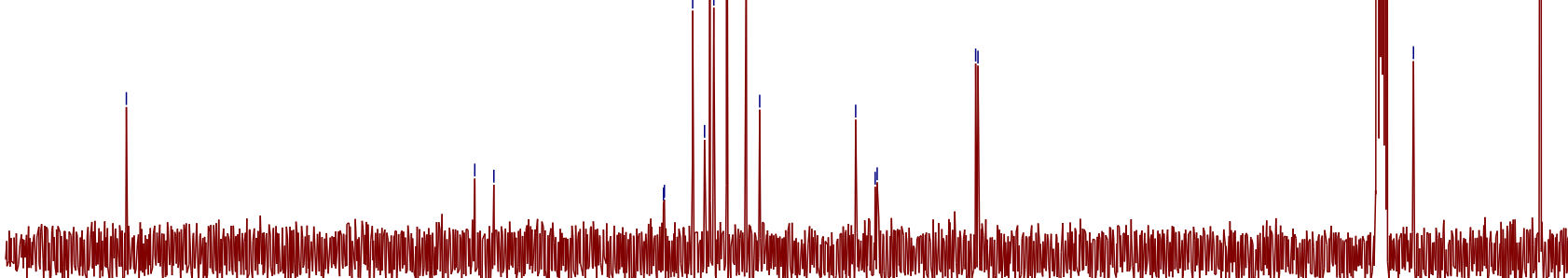

$\begin{array}{lllllll}210 & 200 & 190 & 180 & 170 & 160 & 150\end{array}$

$\begin{array}{lll}140 & 130 & 120\end{array}$

$10 \begin{gathered}100 \\ \mathrm{f} 1(\mathrm{ppm})\end{gathered}$ 


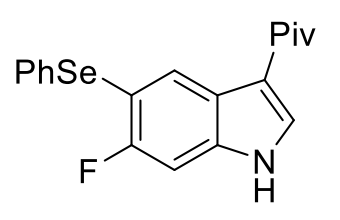

$3 q$

${ }^{19}$ F NMR: $377 \mathrm{MHz}$

Solvent: $\mathrm{CDCl}_{3}$ 
焉

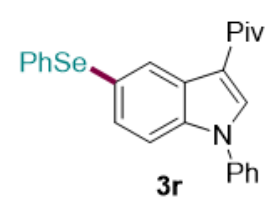

${ }^{1} \mathrm{H}$ NMR: $400 \mathrm{MHz}$

$3 r \quad \mathrm{Ph}$

solvent: $\mathrm{CDCl}_{3}$

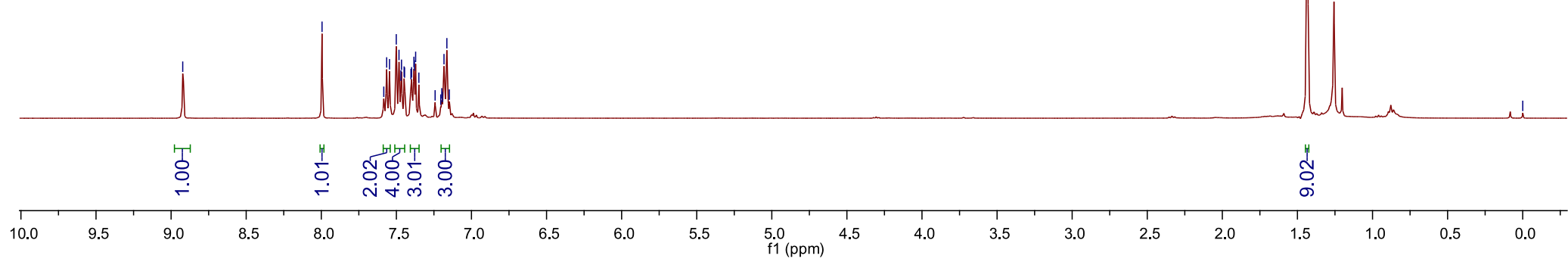




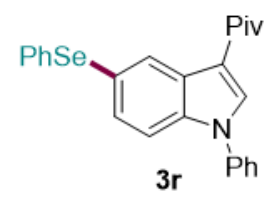

${ }^{13} \mathrm{C}\left\{{ }^{1} \mathrm{H}\right\}$ NMR: $100 \mathrm{MHz}$ solvent: $\mathrm{CDCl}_{3}$

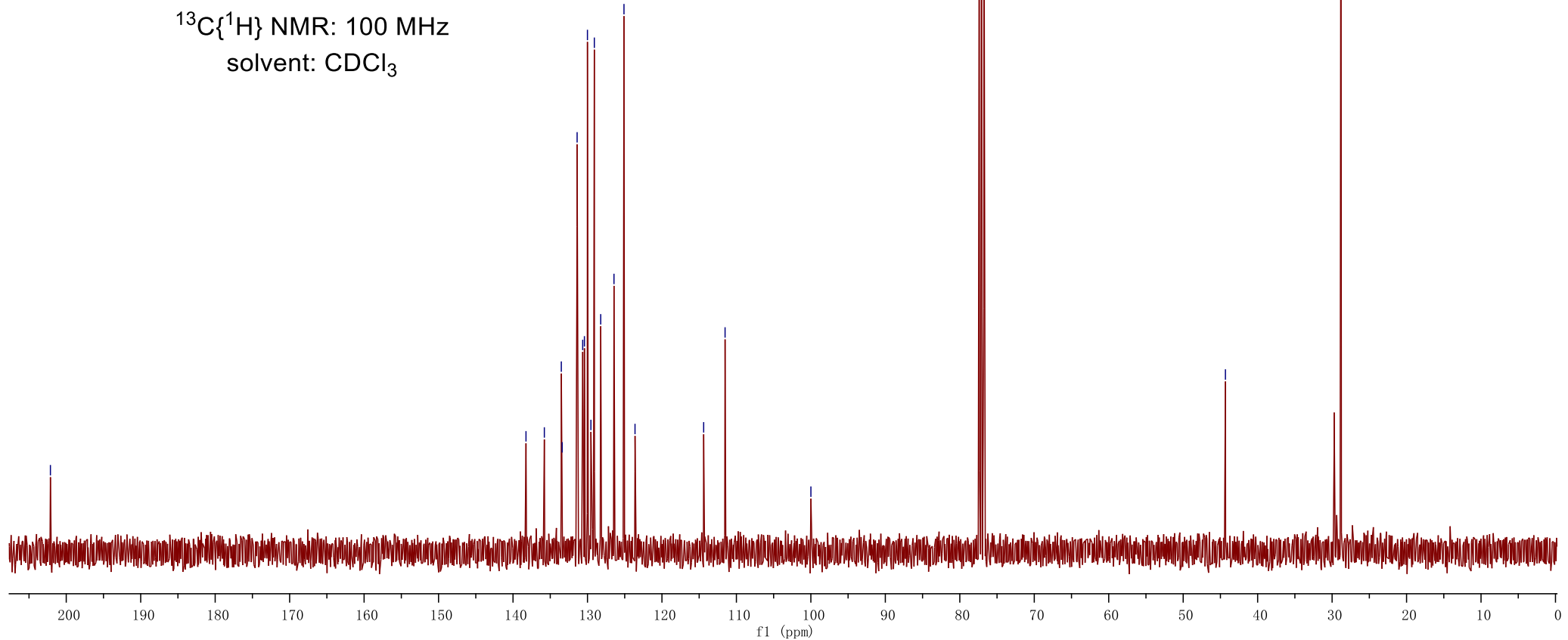




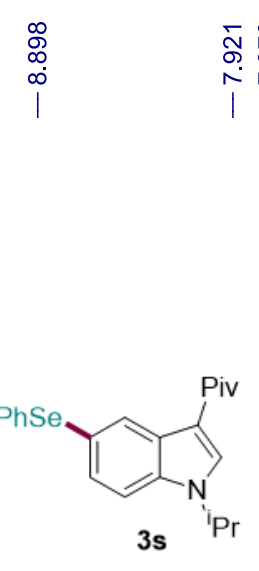

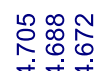

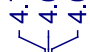

${ }^{1} \mathrm{H}$ NMR: $400 \mathrm{MHz}$

solvent: $\mathrm{CDCl}_{3}$

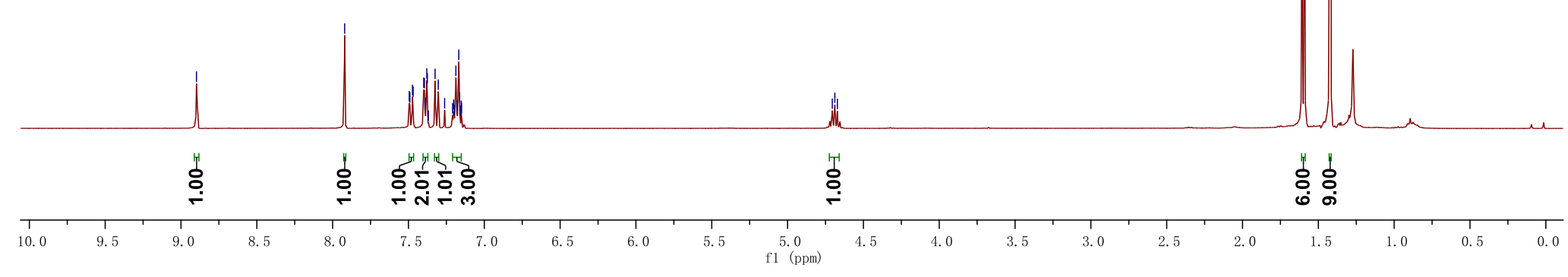




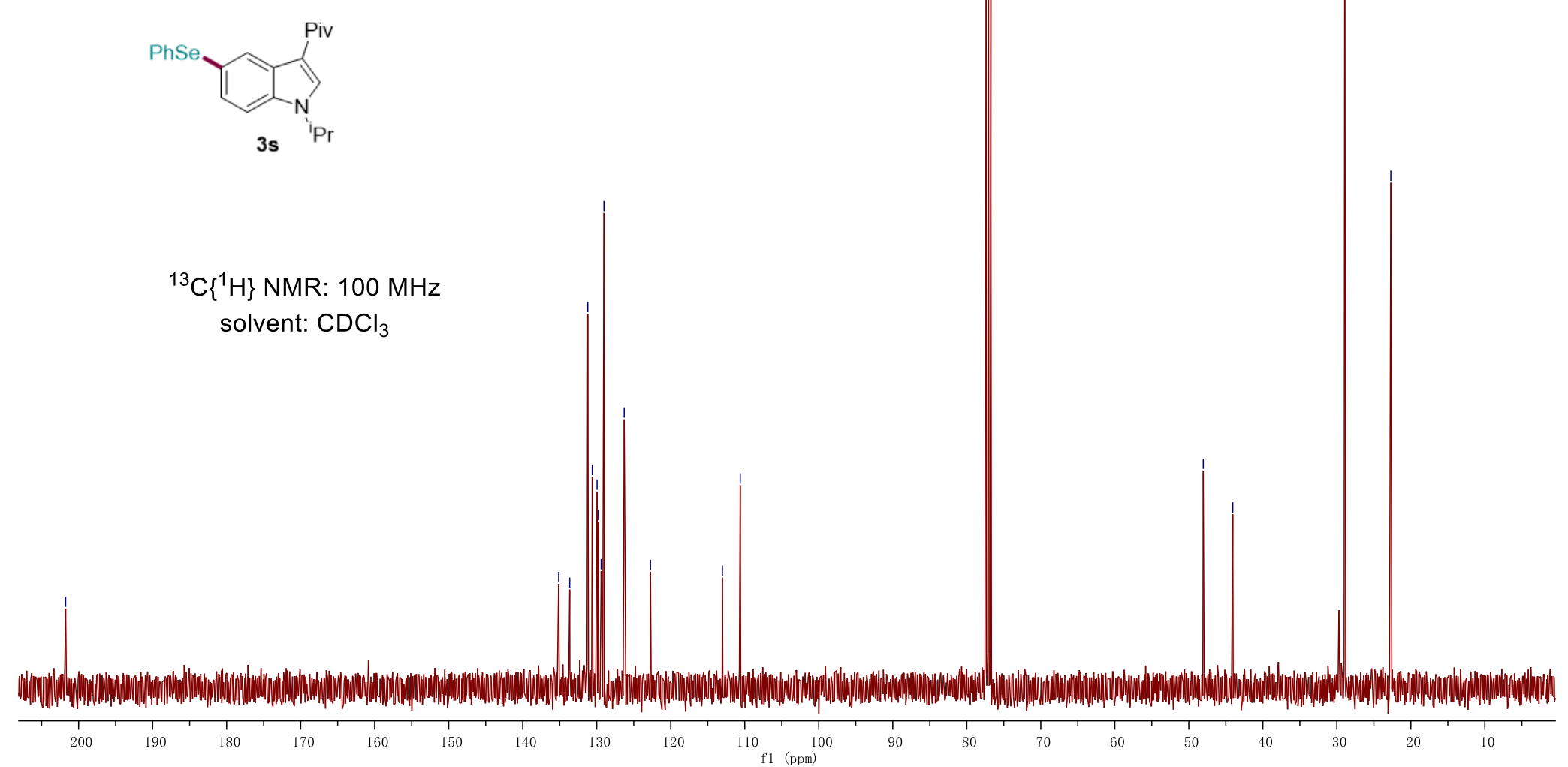




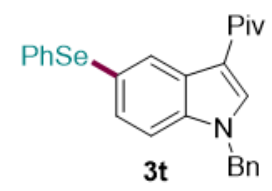

${ }^{1} \mathrm{H}$ NMR: $400 \mathrm{MHz}$

solvent: $\mathrm{CDCl}_{3}$ 


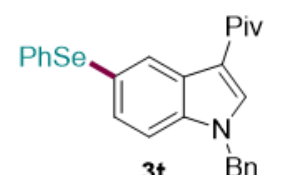

3t

${ }^{13} \mathrm{C}\left\{{ }^{1} \mathrm{H}\right\}$ NMR: $100 \mathrm{MHz}$ solvent: $\mathrm{CDCl}_{3}$ 


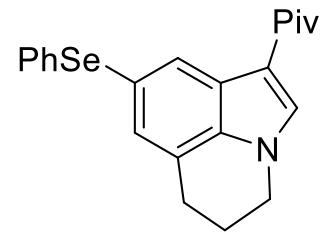

$3 u$

${ }^{1} \mathrm{H}$ NMR: $400 \mathrm{MHz}$

solvent: $\mathrm{CDCl}_{3}$

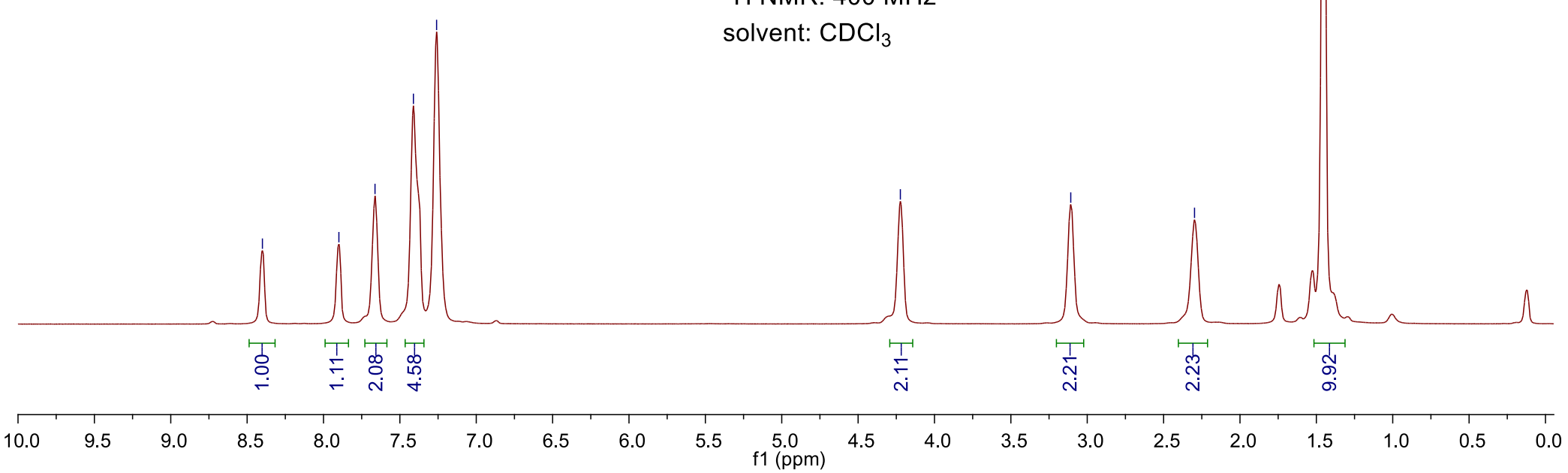




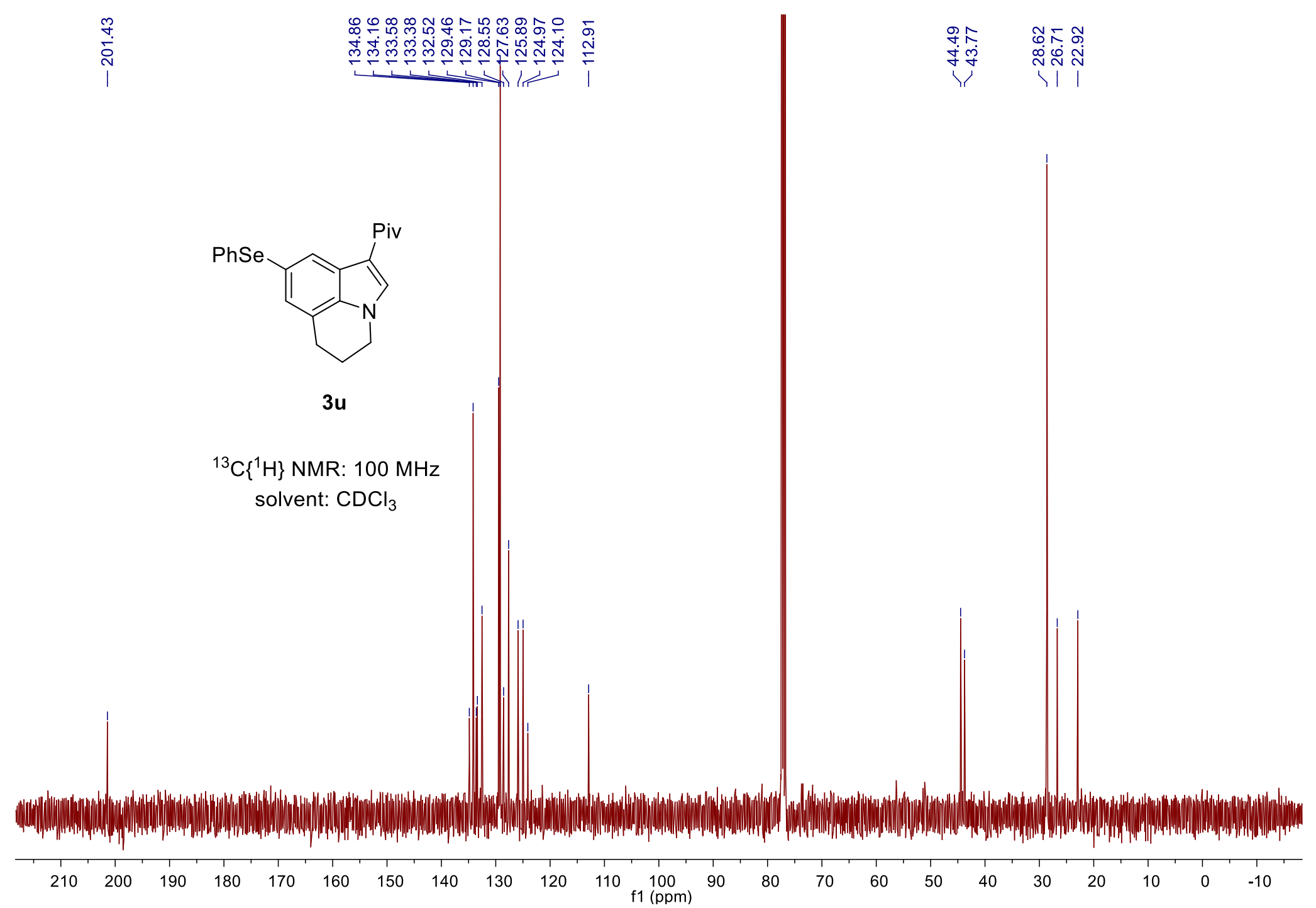




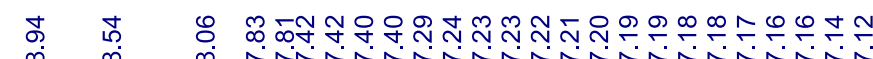

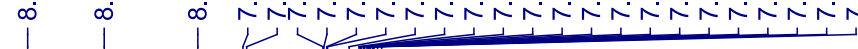

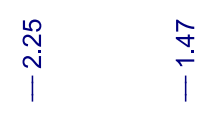

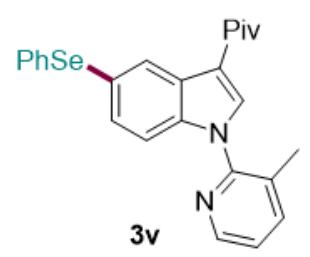

${ }^{1} \mathrm{H}$ NMR: $400 \mathrm{MHz}$

solvent: $\mathrm{CDCl}_{3}$

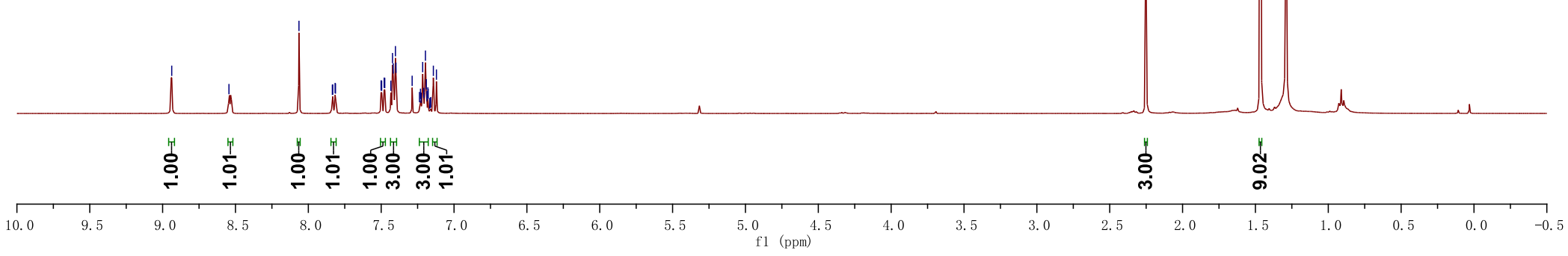




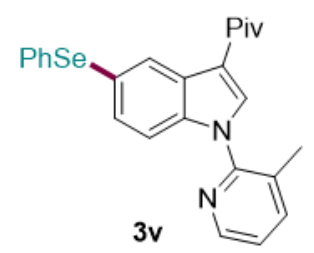

${ }^{13} \mathrm{C}\left\{{ }^{1} \mathrm{H}\right\}$ NMR: $100 \mathrm{MHz}$ solvent: $\mathrm{CDCl}_{3}$

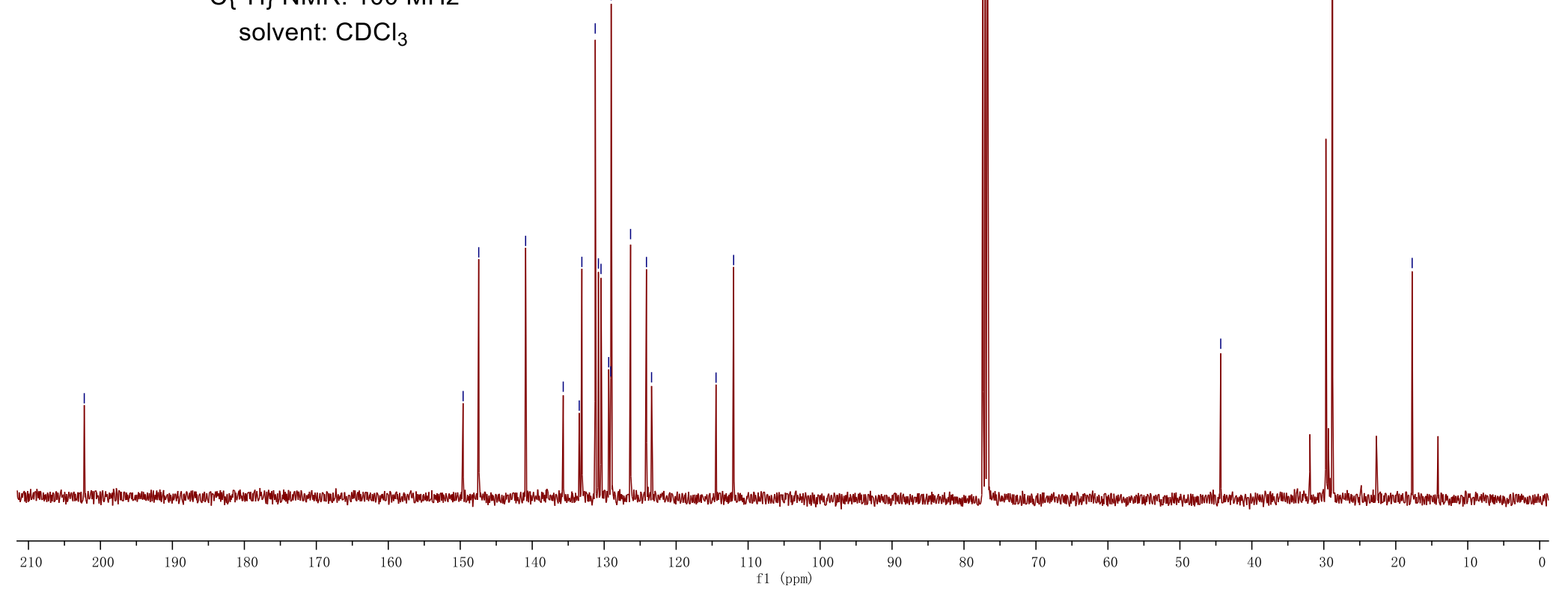




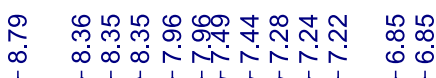

$\checkmark 4$

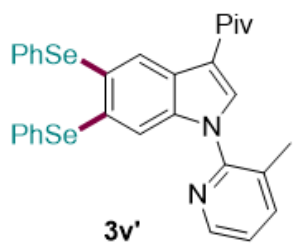

${ }^{1} \mathrm{H}$ NMR: $400 \mathrm{MHz}$

solvent: $\mathrm{CDCl}_{3}$

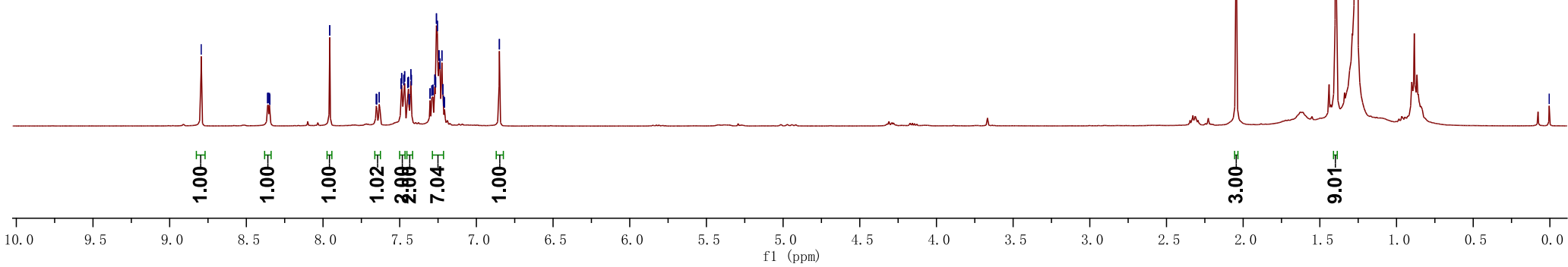




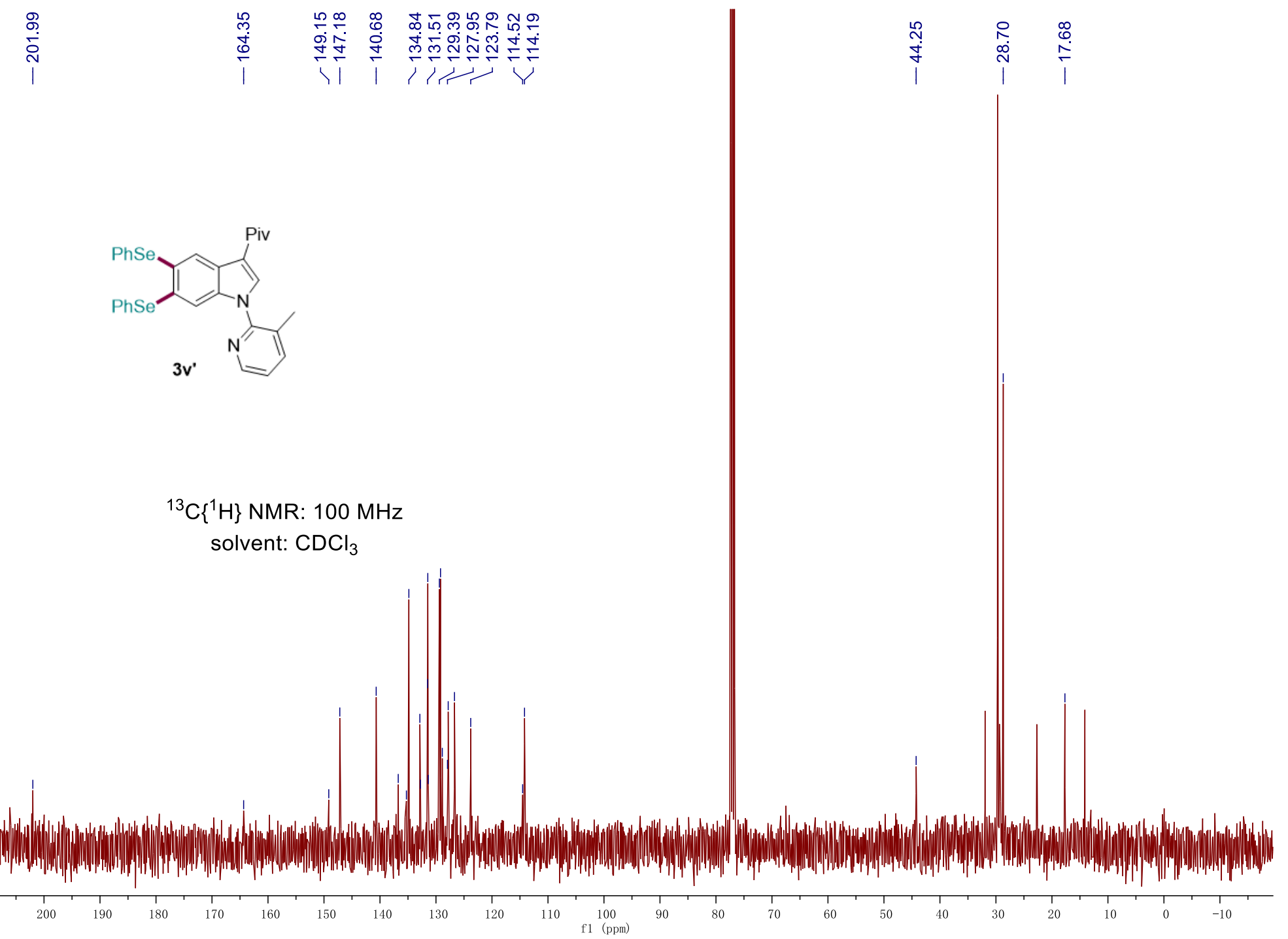




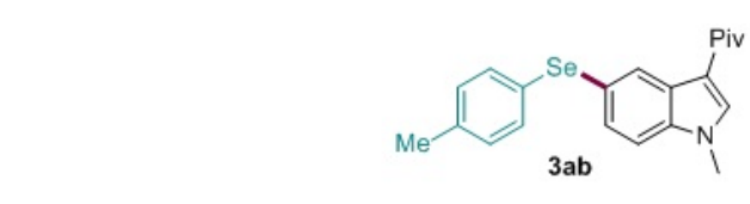

${ }^{1} \mathrm{H}$ NMR: $400 \mathrm{MHz}$

solvent: $\mathrm{CDCl}_{3}$

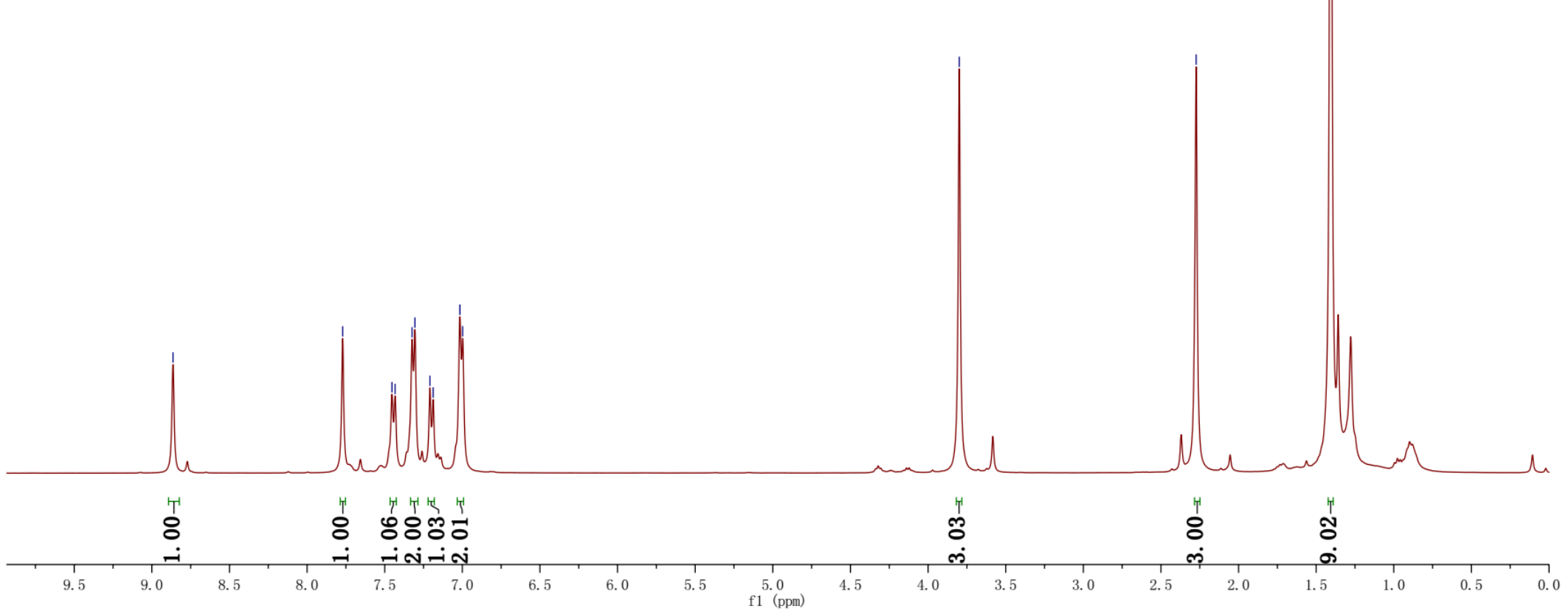




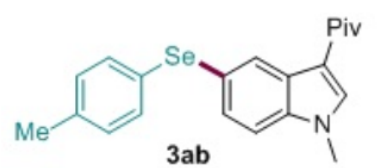

3ab

${ }^{13} \mathrm{C}\left\{{ }^{1} \mathrm{H}\right\}$ NMR: $100 \mathrm{MHz}$

solvent: $\mathrm{CDCl}_{3}$

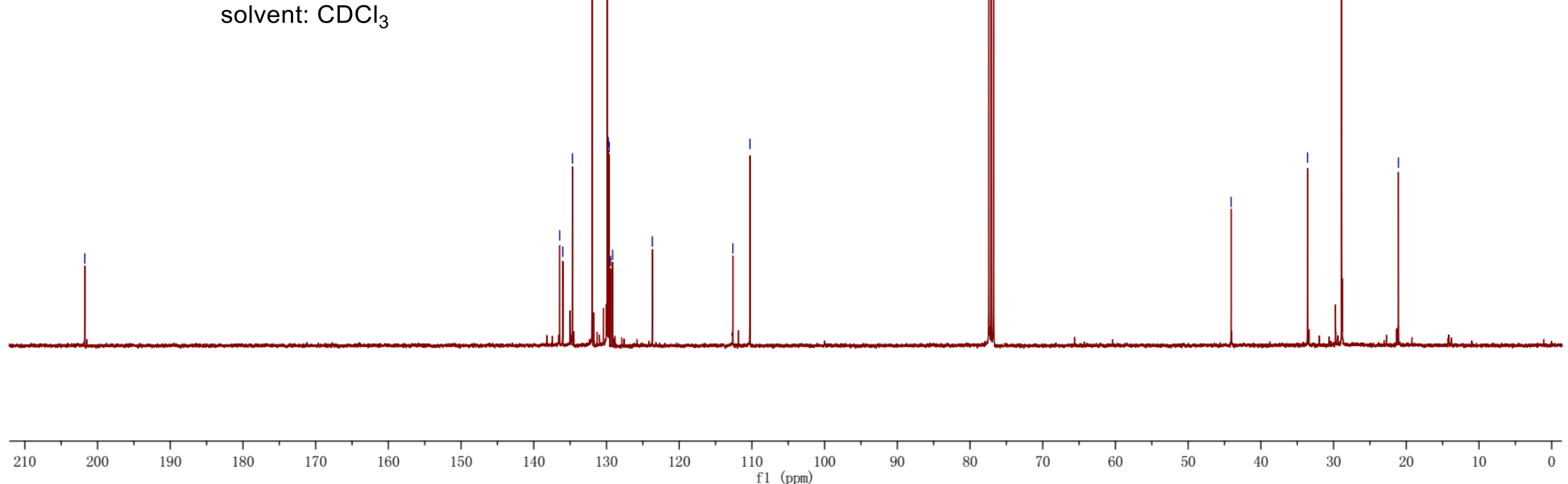




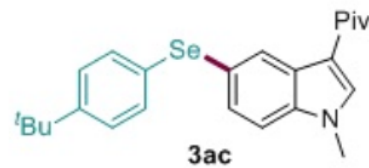

${ }^{1} \mathrm{H}$ NMR: $400 \mathrm{MHz}$

solvent: $\mathrm{CDCl}_{3}$

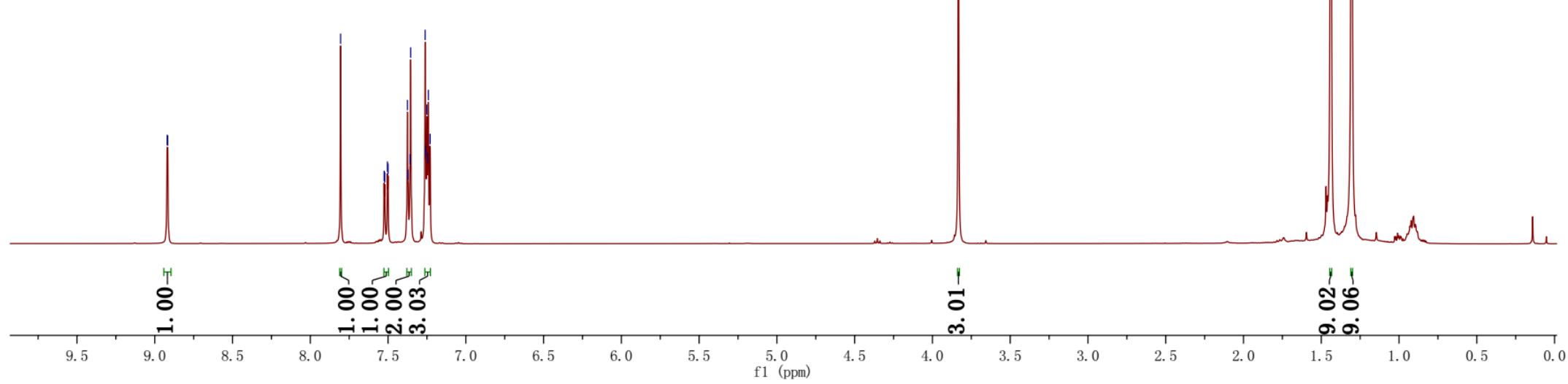




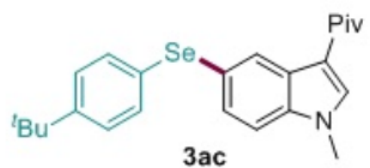

${ }^{13} \mathrm{C}\left\{{ }^{1} \mathrm{H}\right\}$ NMR: $100 \mathrm{MHz}$ solvent: $\mathrm{CDCl}_{3}$ 


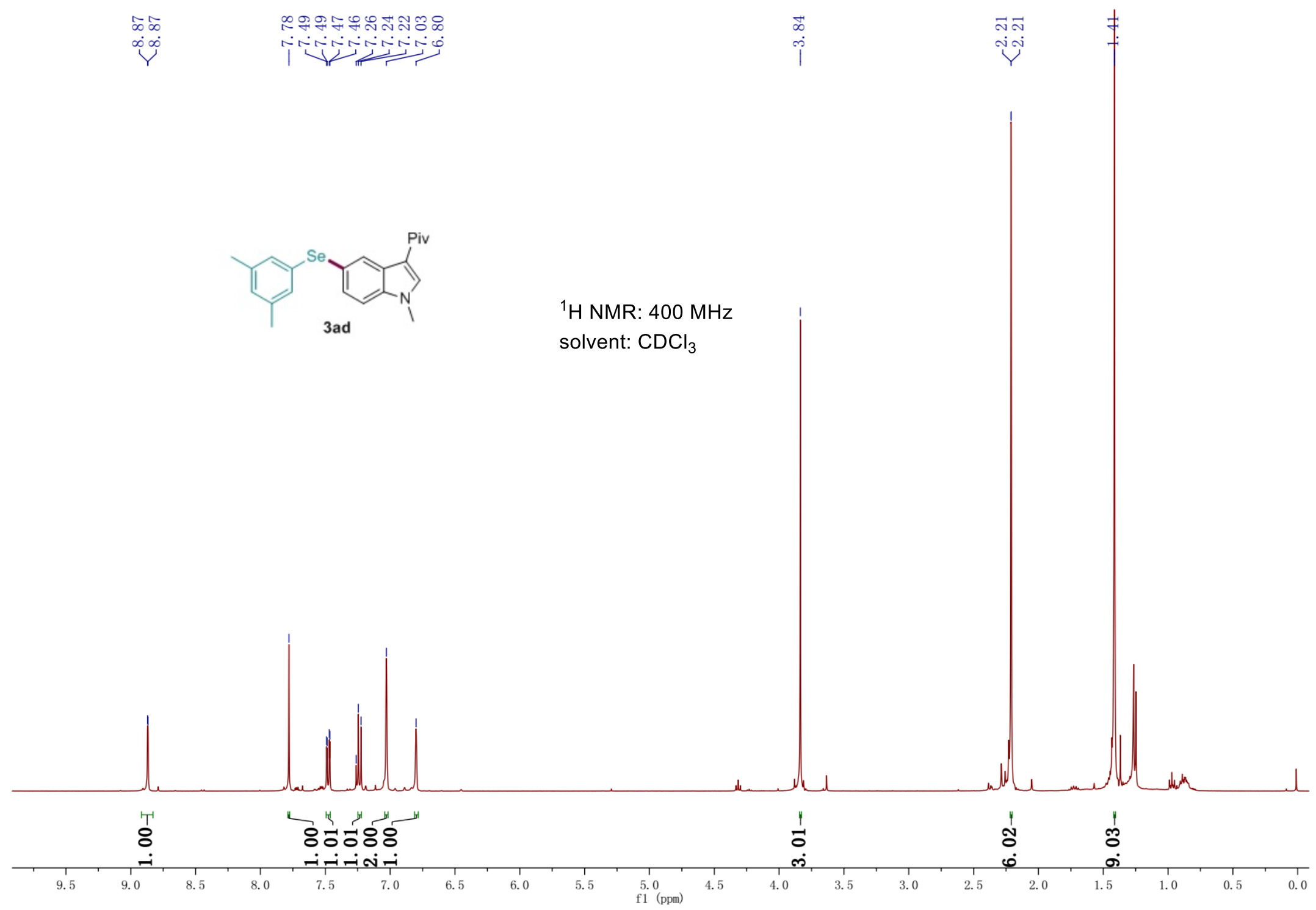




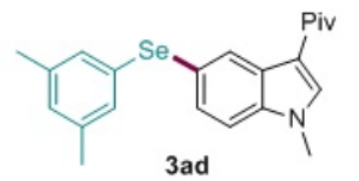

3ad

${ }^{13} \mathrm{C}\left\{{ }^{1} \mathrm{H}\right\}$ NMR: $100 \mathrm{MHz}$ solvent: $\mathrm{CDCl}_{3}$

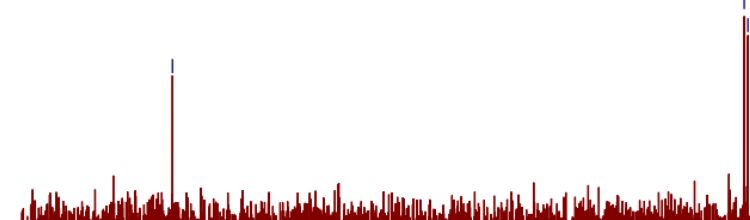

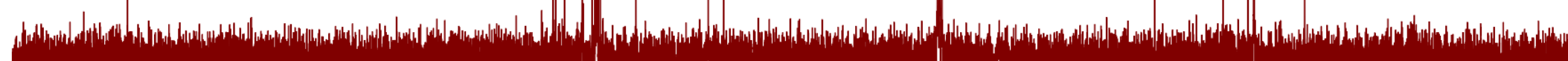

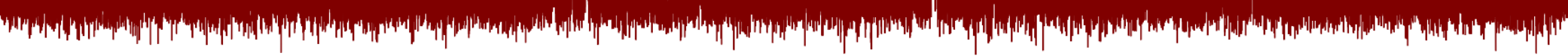

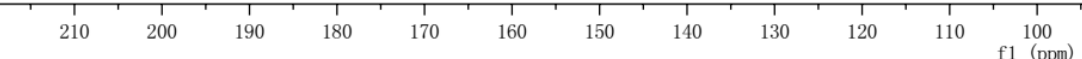

100

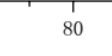

$70 \quad 60$

$50 \quad 40$

30 

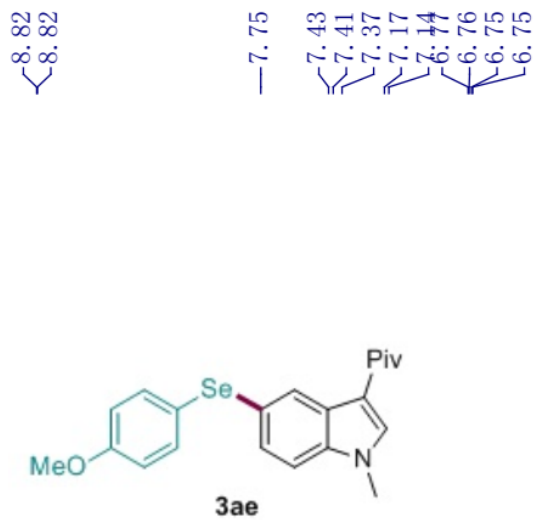

$3 a e$

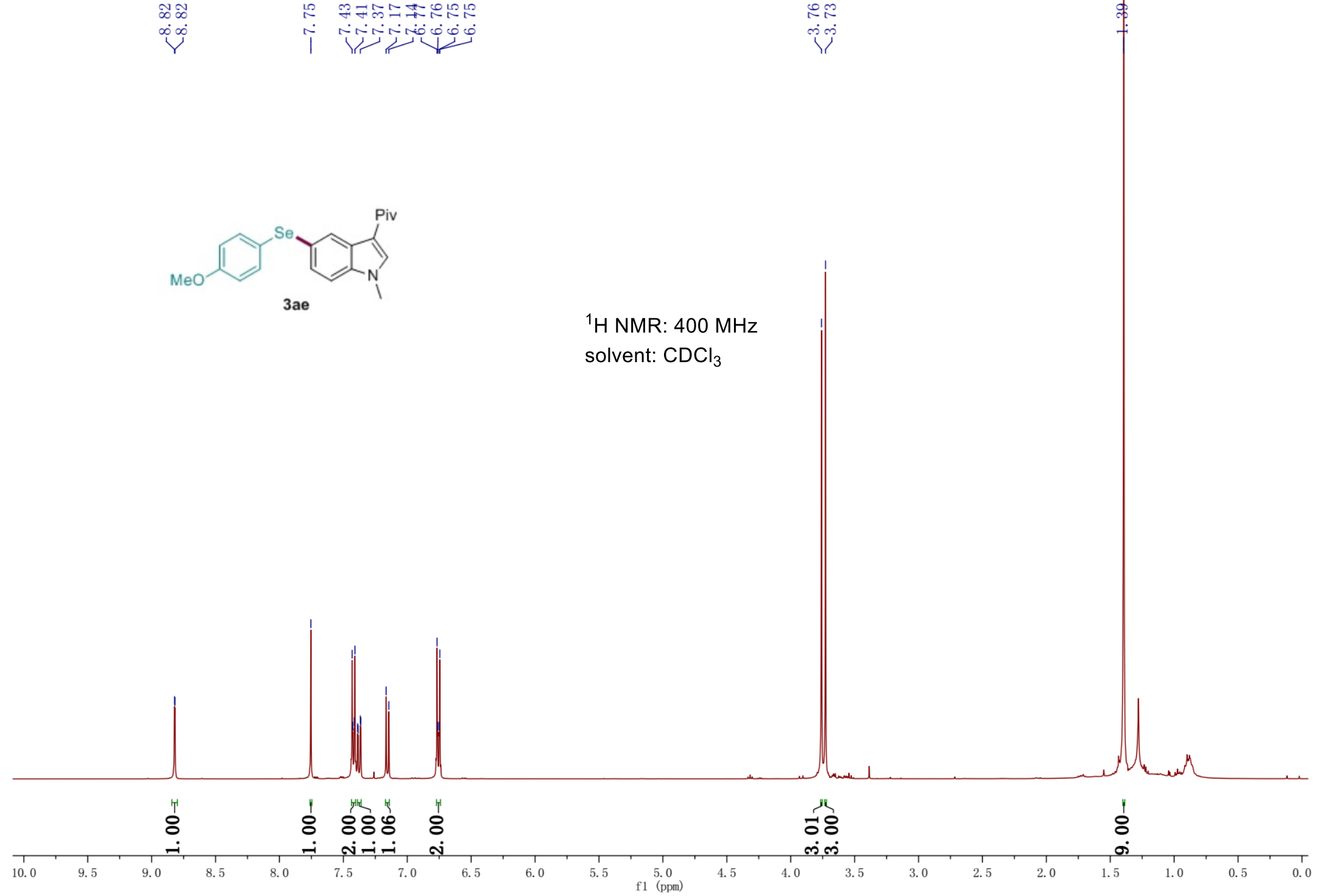

유 


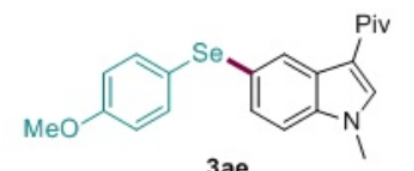

3 ae

${ }^{13} \mathrm{C}\left\{{ }^{1} \mathrm{H}\right\}$ NMR: $100 \mathrm{MHz}$ solvent: $\mathrm{CDCl}_{3}$

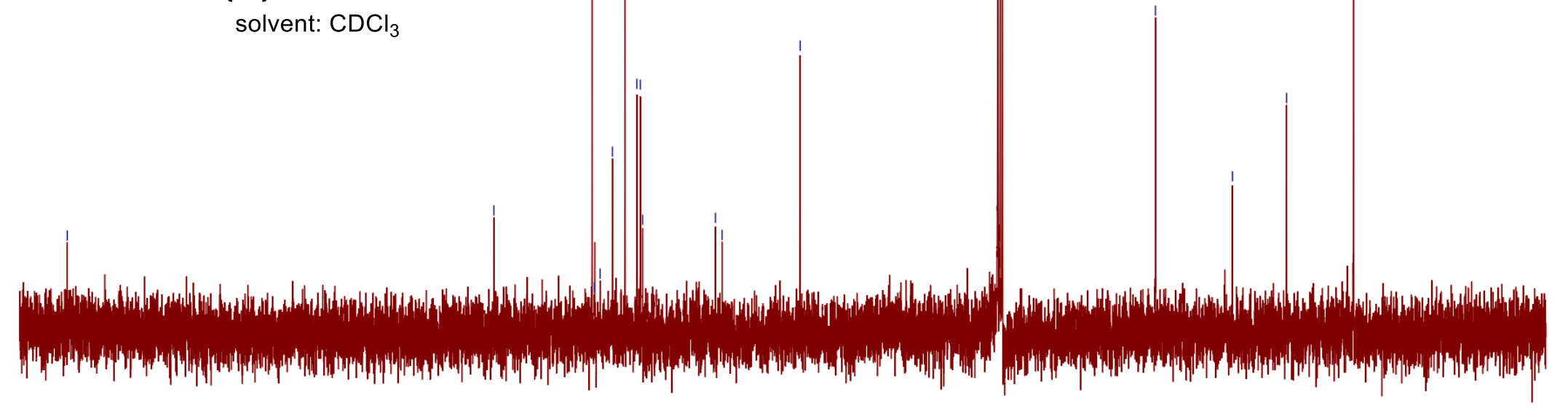

$$
\frac{1}{210}
$$
$200 \quad 190$ 1
$90 \quad 180$ 170 160 $150 \quad 140$ $\underset{130}{1} \quad 120$ ${ }_{\text {f1 }}^{110} 100$ 

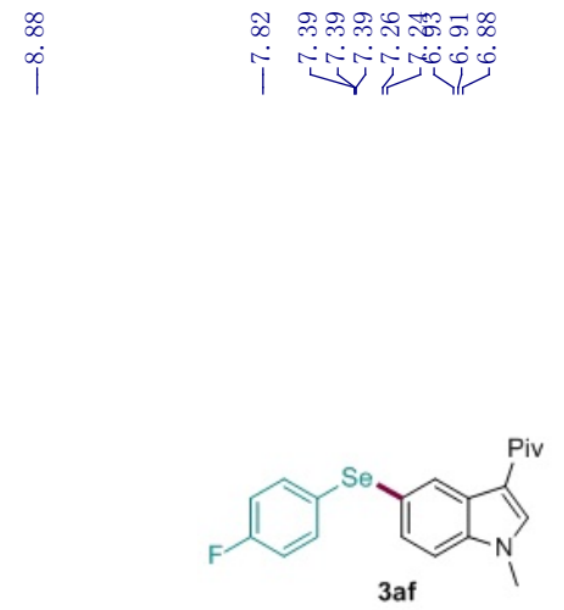

3af

${ }^{1} \mathrm{H}$ NMR: $400 \mathrm{MHz}$

solvent: $\mathrm{CDCl}_{3}$

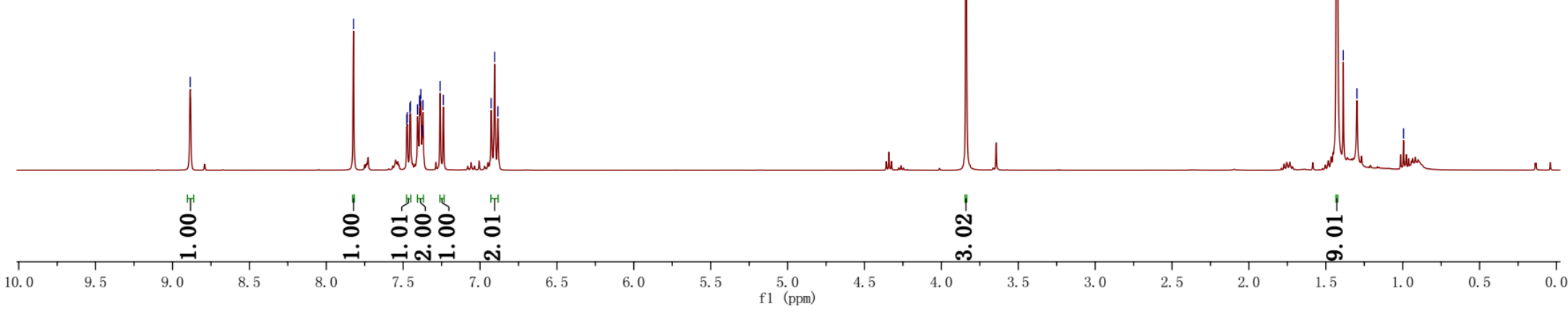




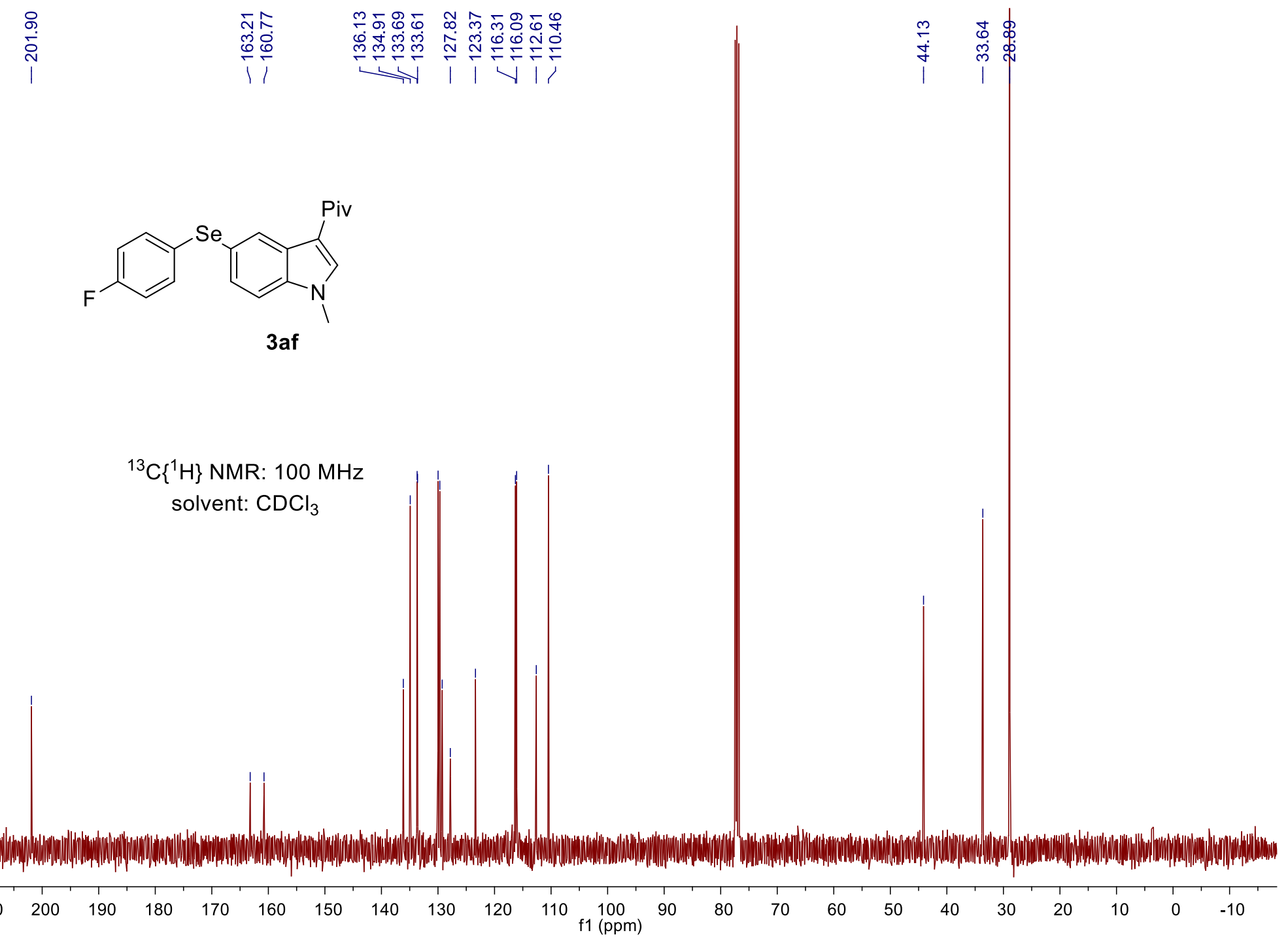




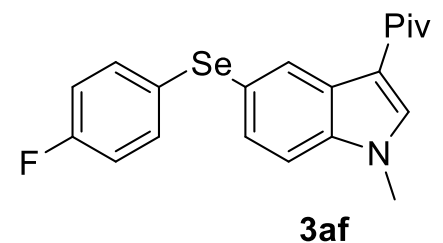

${ }^{19} \mathrm{~F}$ NMR: $377 \mathrm{MHz}$

Solvent: $\mathrm{CDCl}_{3}$ 


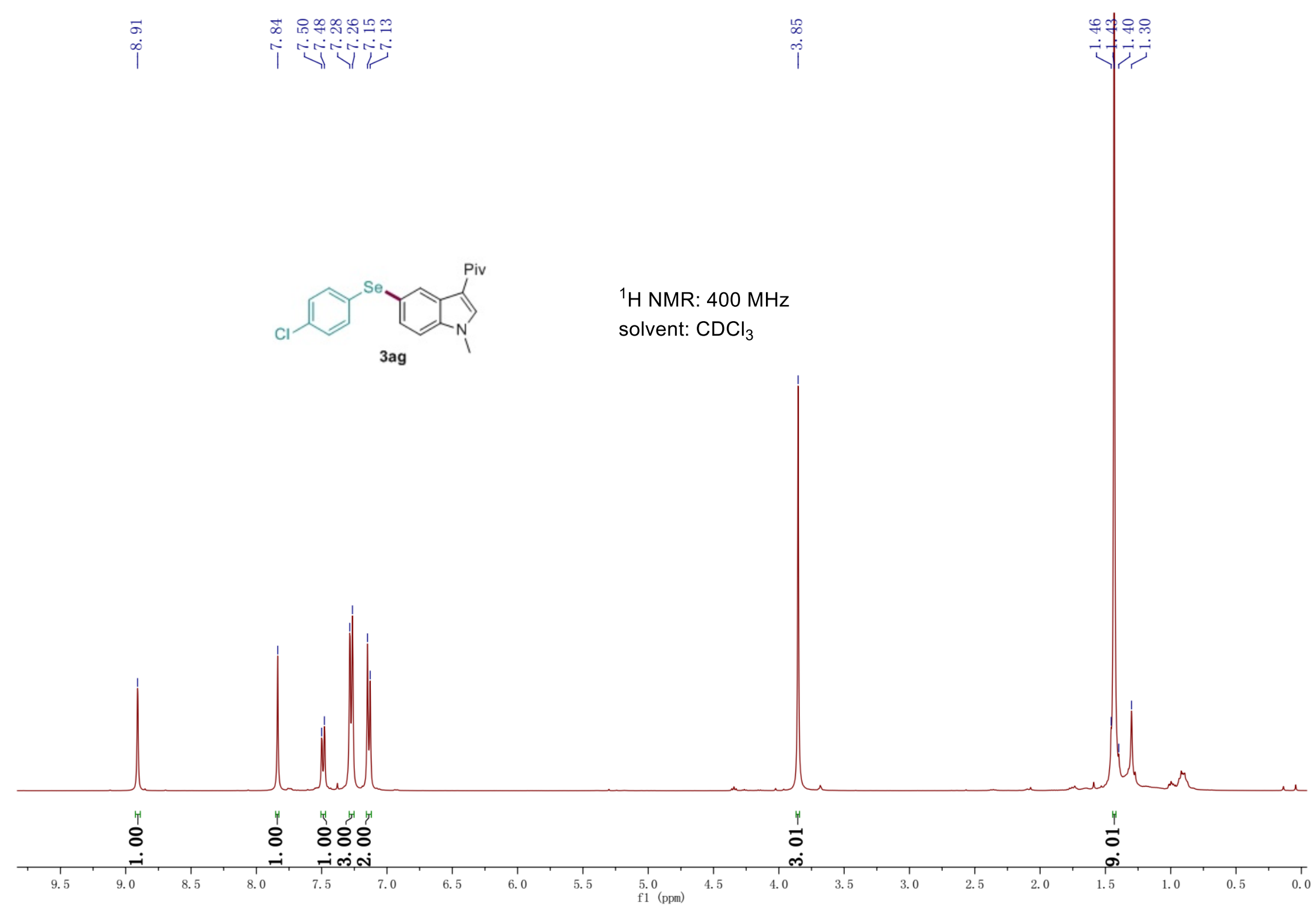




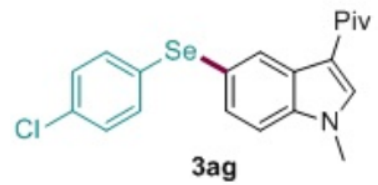

${ }^{13} \mathrm{C}\left\{{ }^{1} \mathrm{H}\right\}$ NMR: $100 \mathrm{MHz}$ solvent: $\mathrm{CDCl}_{3}$
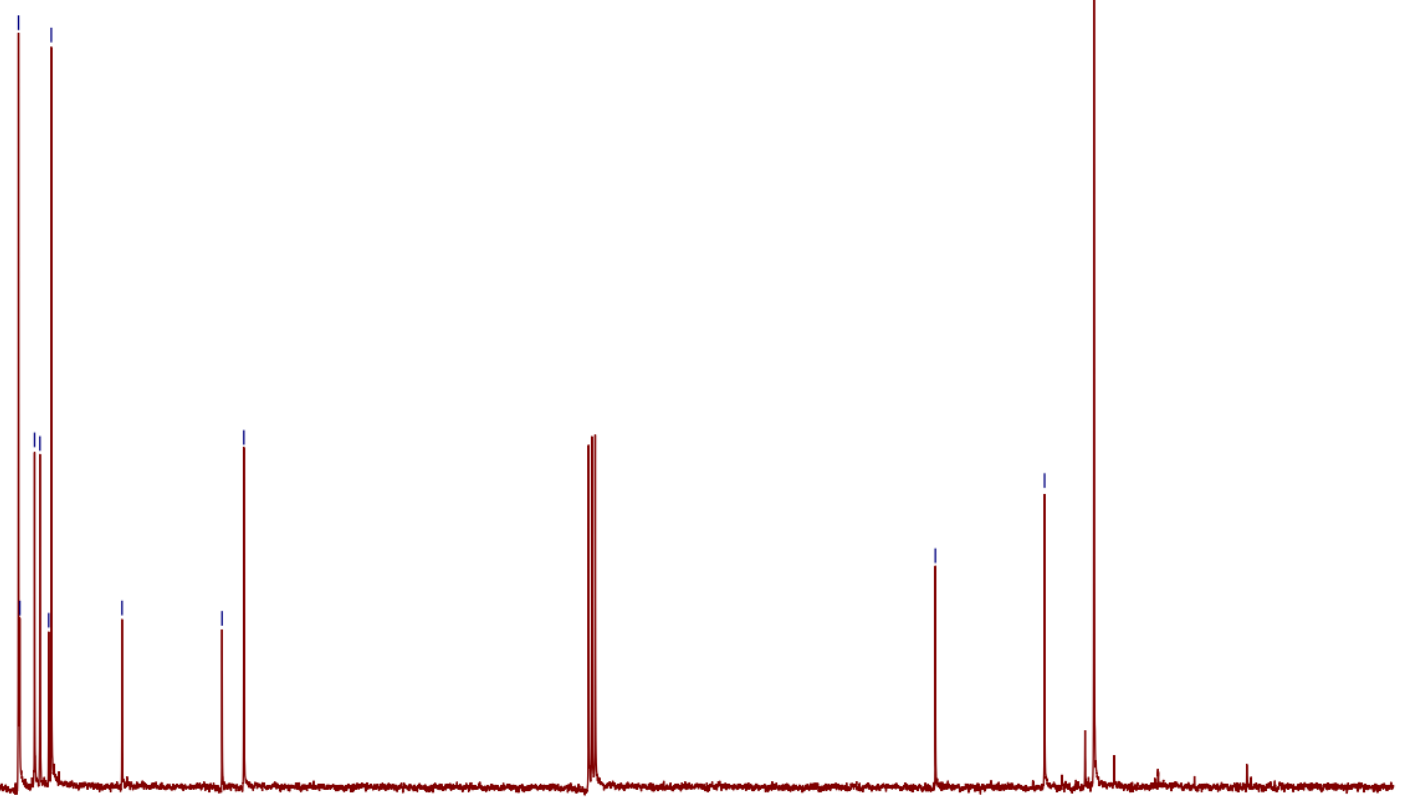

$200 \quad 190$

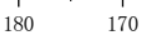

$160 \quad 150$

$140 \quad 130$ 


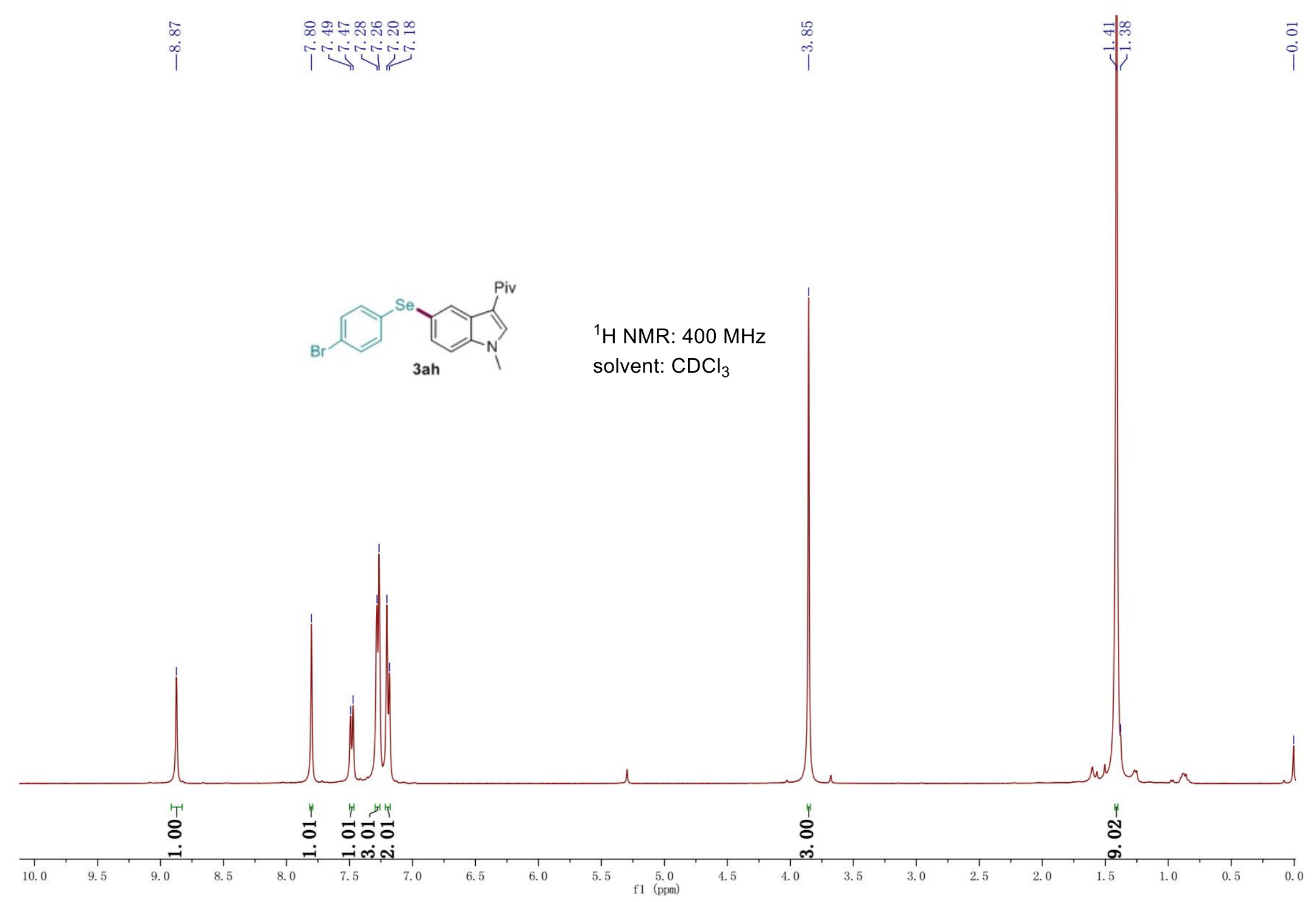




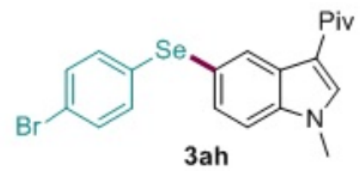

${ }^{13} \mathrm{C}\left\{{ }^{1} \mathrm{H}\right\}$ NMR: $100 \mathrm{MHz}$ solvent: $\mathrm{CDCl}_{3}$

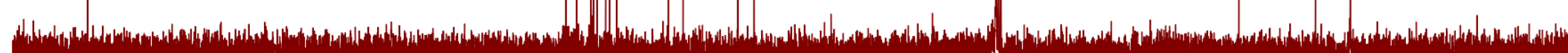

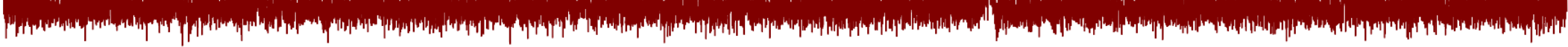

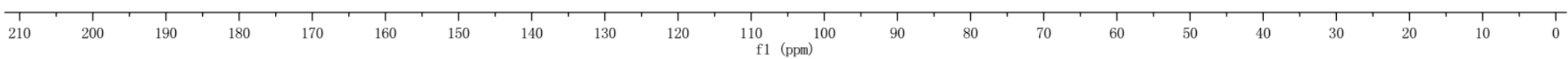




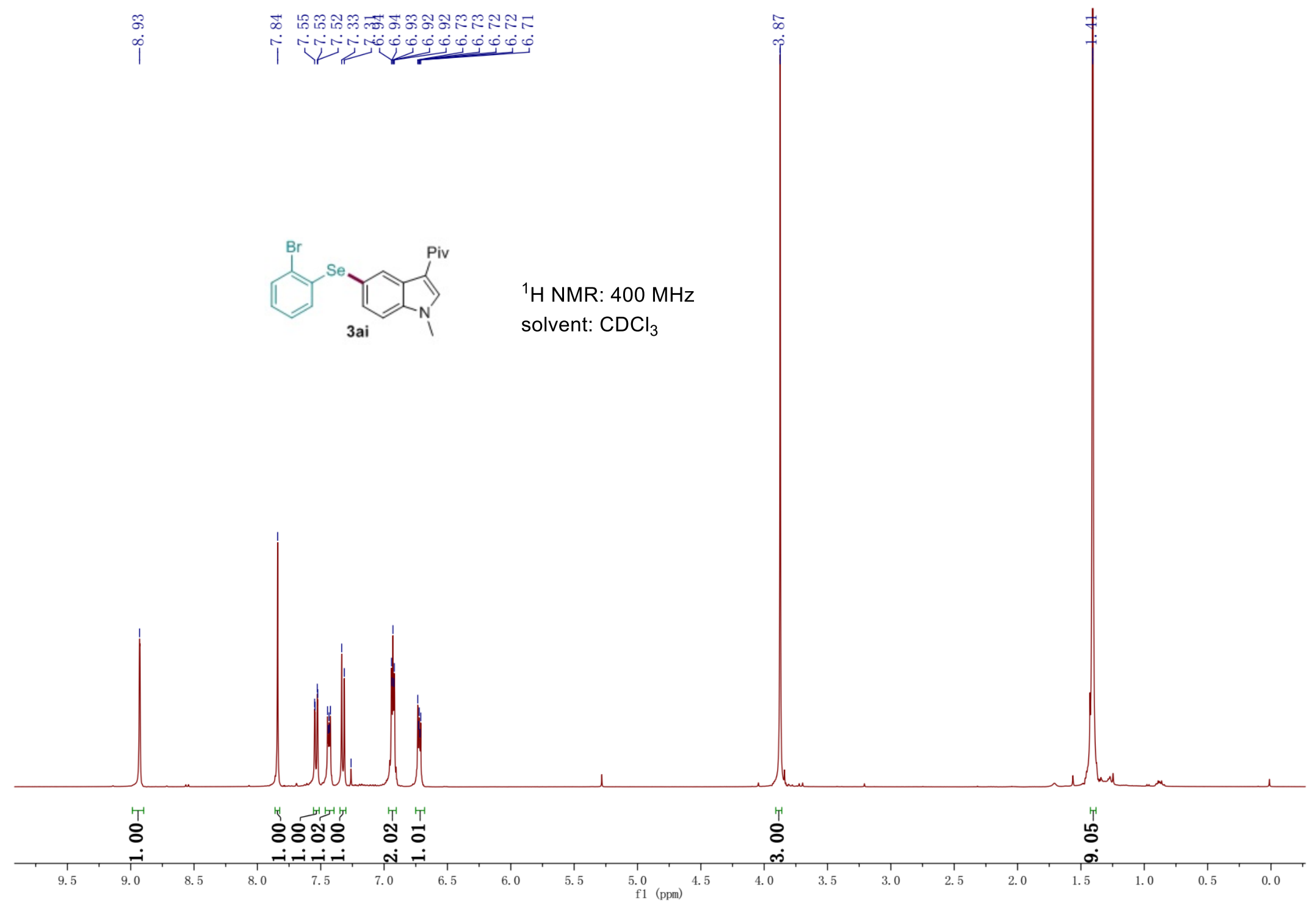




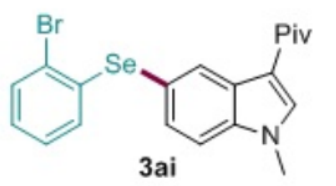

${ }^{13} \mathrm{C}\left\{{ }^{1} \mathrm{H}\right\}$ NMR: $100 \mathrm{MHz}$ solvent: $\mathrm{CDCl}_{3}$

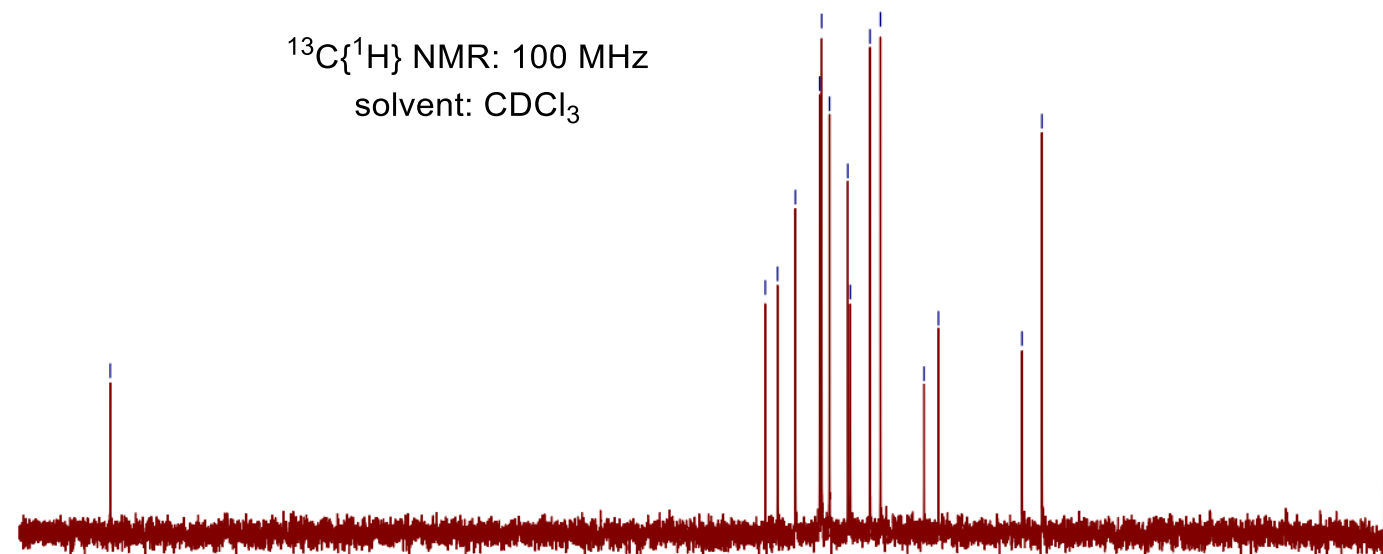




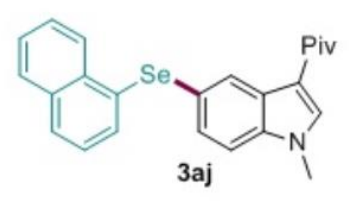

${ }^{1} \mathrm{H}$ NMR: $400 \mathrm{MHz}$ solvent: $\mathrm{CDCl}_{3}$

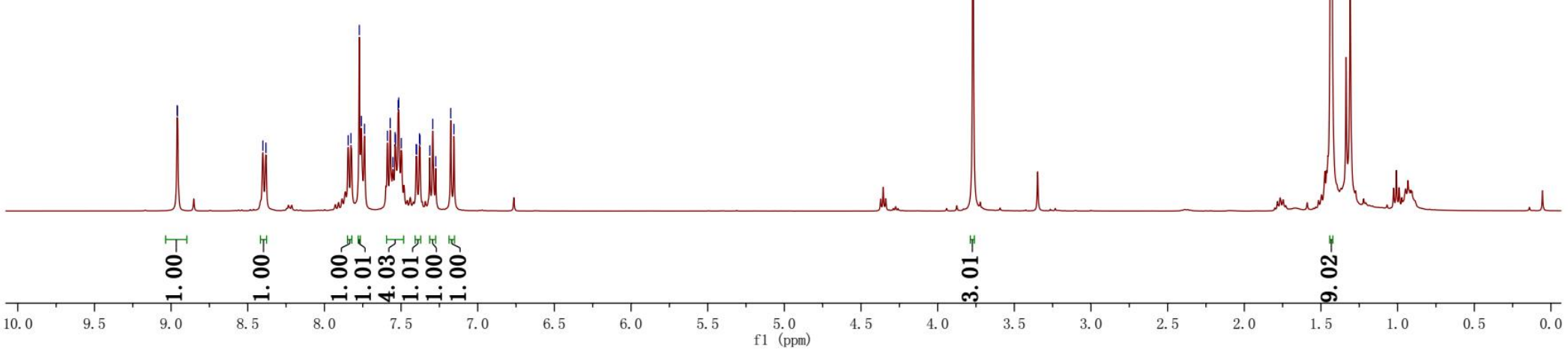



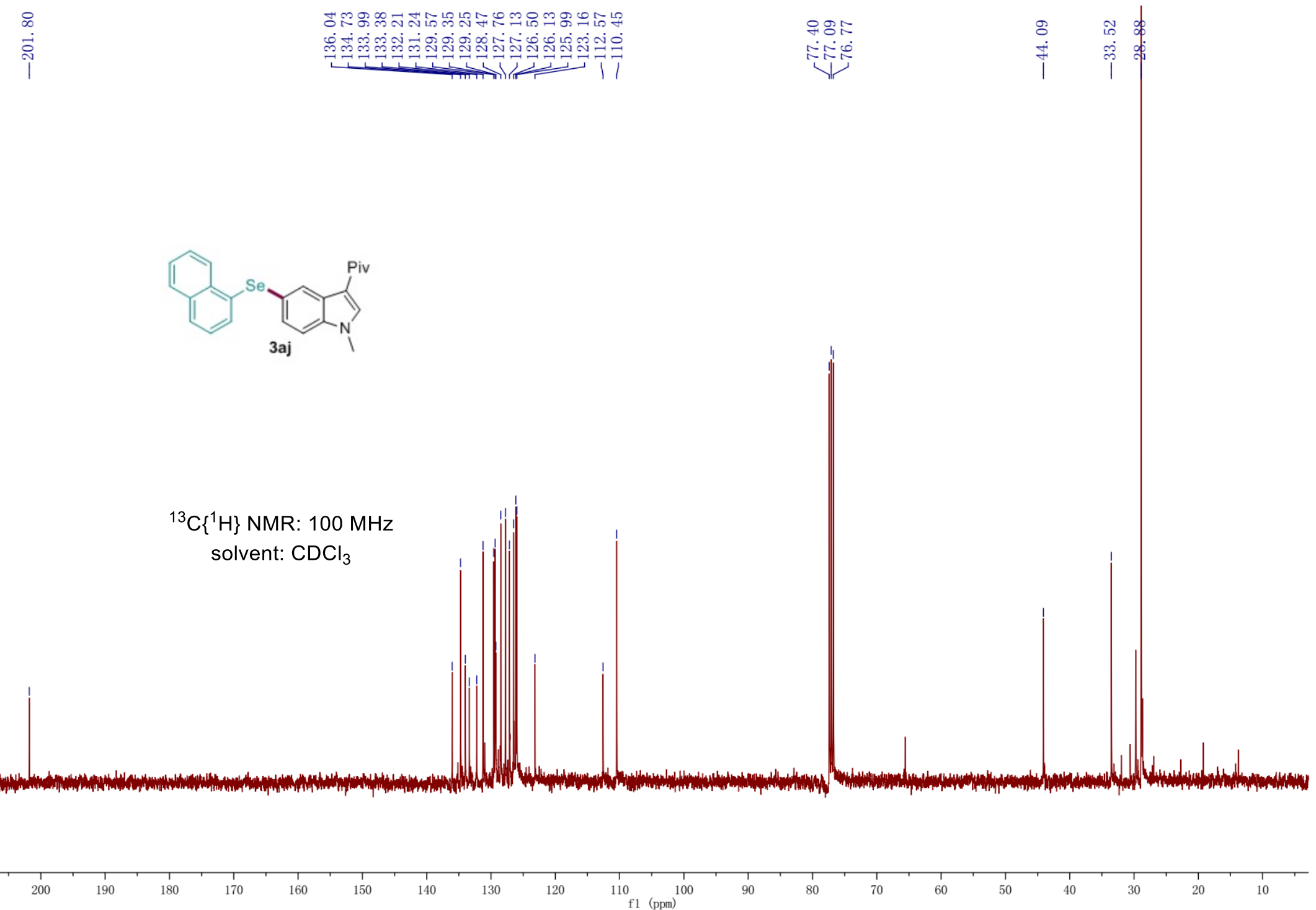


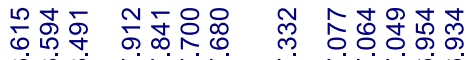

र्रिं0

प) से

1

3ak

${ }^{1} \mathrm{H}$ NMR: $400 \mathrm{MHz}$

solvent: $\mathrm{CDCl}_{3}$

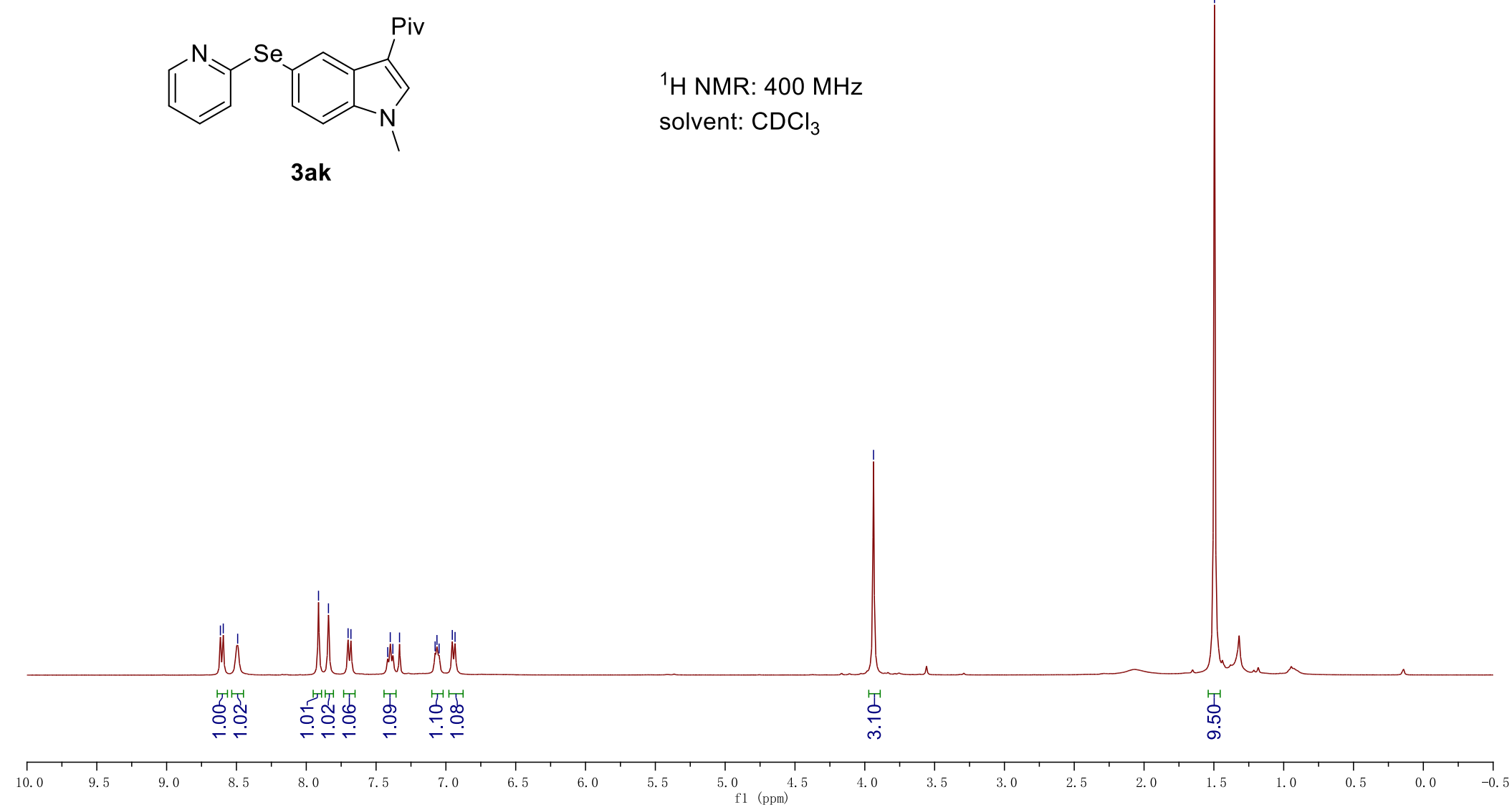




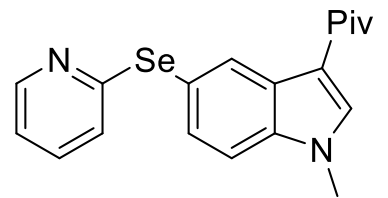

3ak

${ }^{13} \mathrm{C}\left\{{ }^{1} \mathrm{H}\right\}$ NMR: $100 \mathrm{MHz}$ solvent: $\mathrm{CDCl}_{3}$

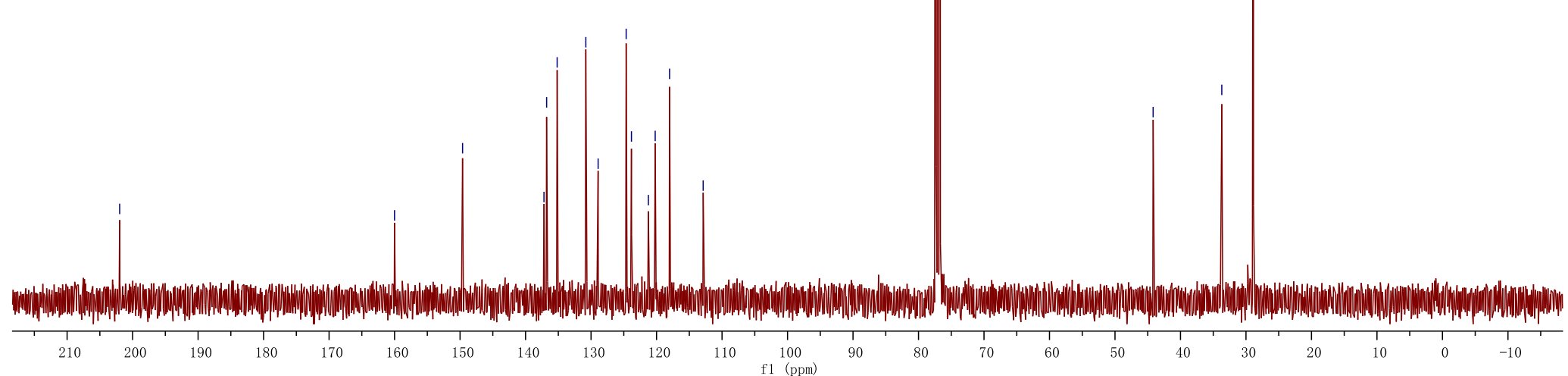




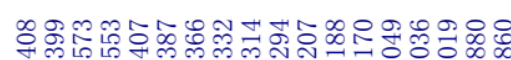

o

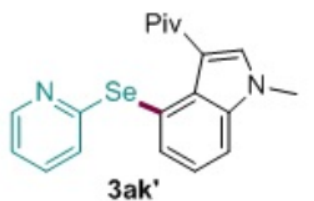

3ak'
${ }^{1} \mathrm{H}$ NMR: $400 \mathrm{MHz}$

solvent: $\mathrm{CDCl}_{3}$

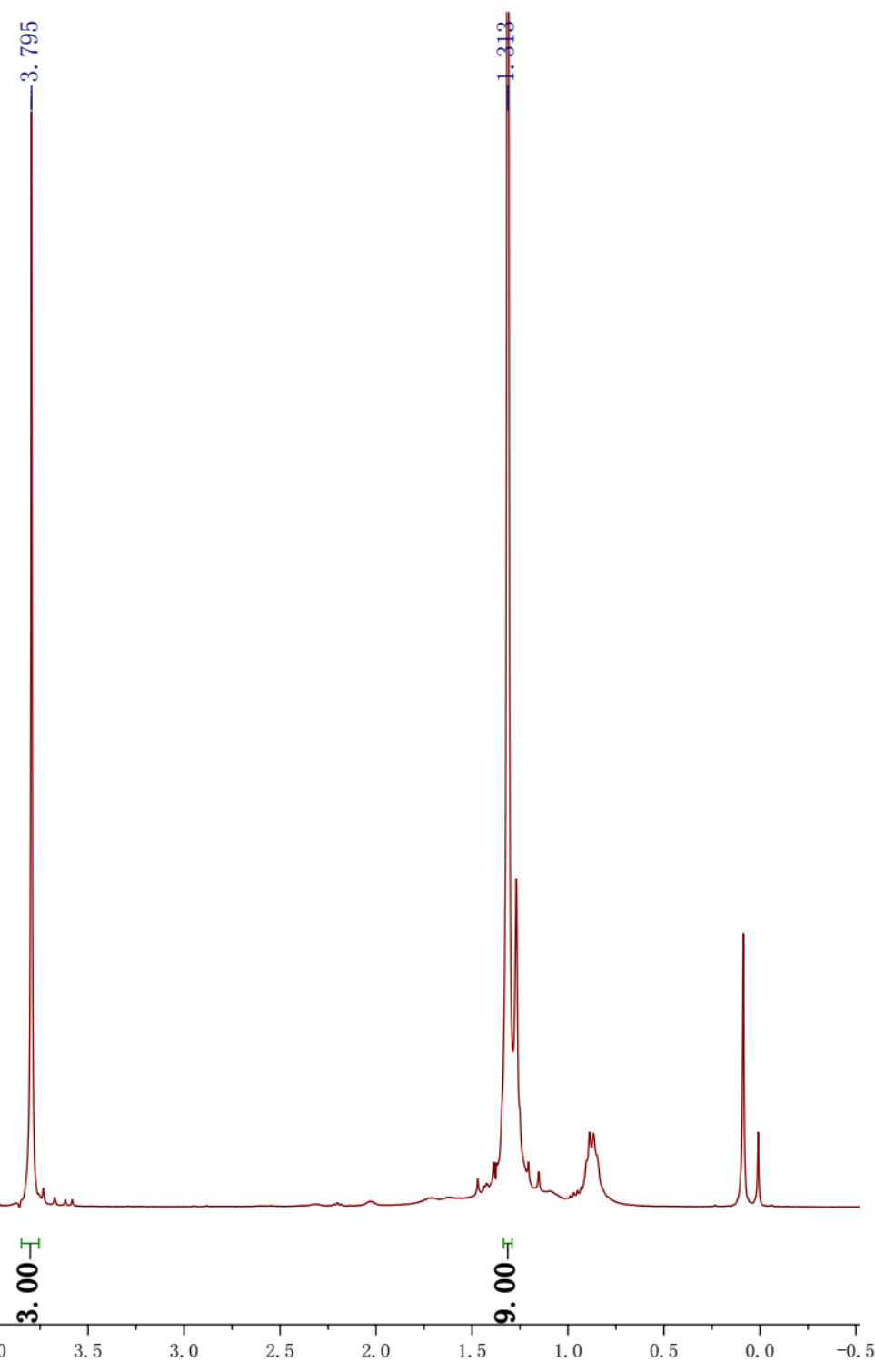




$$
=1
$$


${ }^{1} \mathrm{H}$ NMR: $400 \mathrm{MHz}$

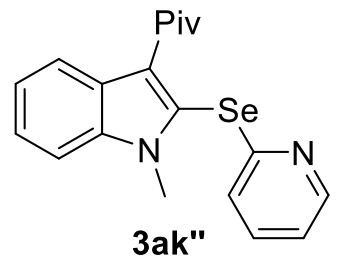

solvent: $\mathrm{CDCl}_{3}$

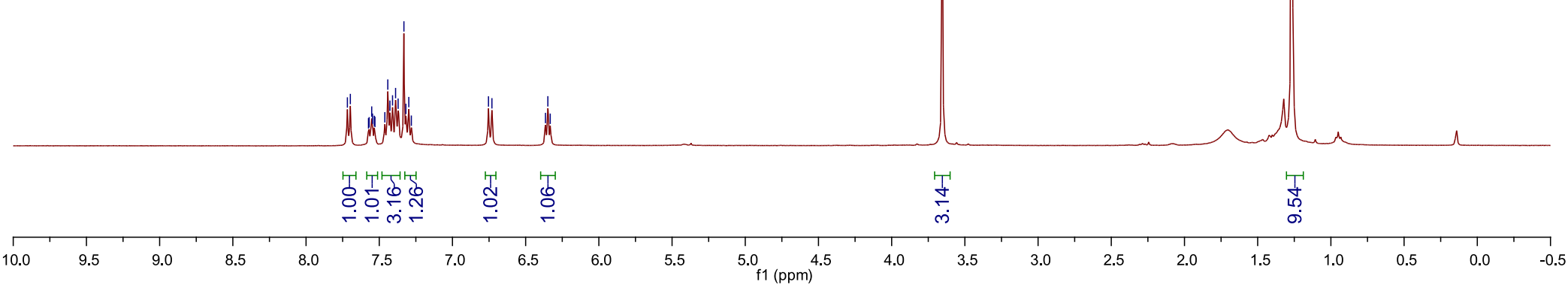




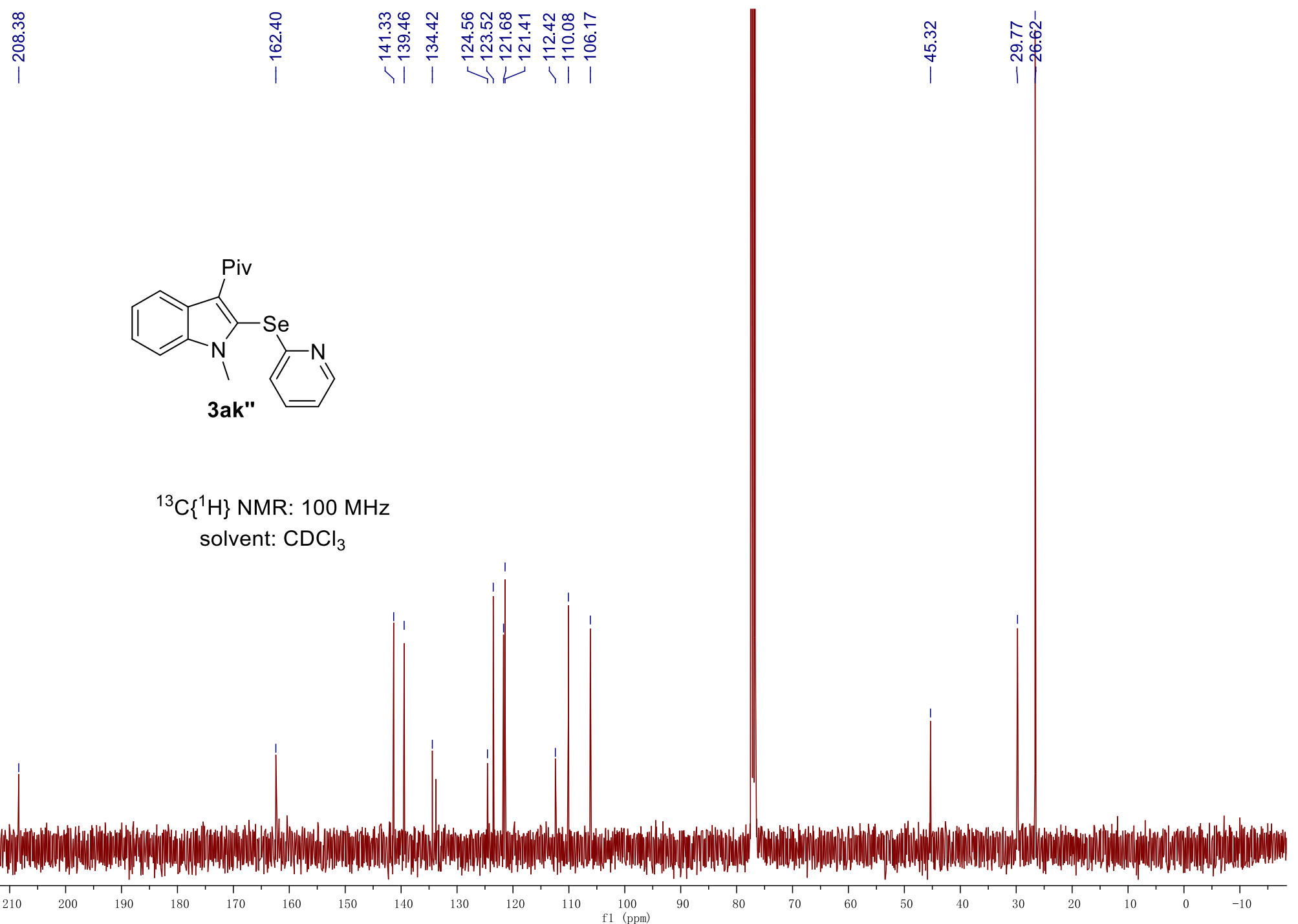




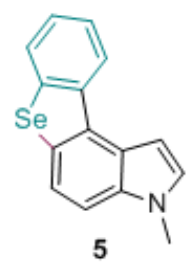

\section{${ }^{1} \mathrm{H}$ NMR: $600 \mathrm{MHz}$}

solvent: $\mathrm{CDCl}_{3}$

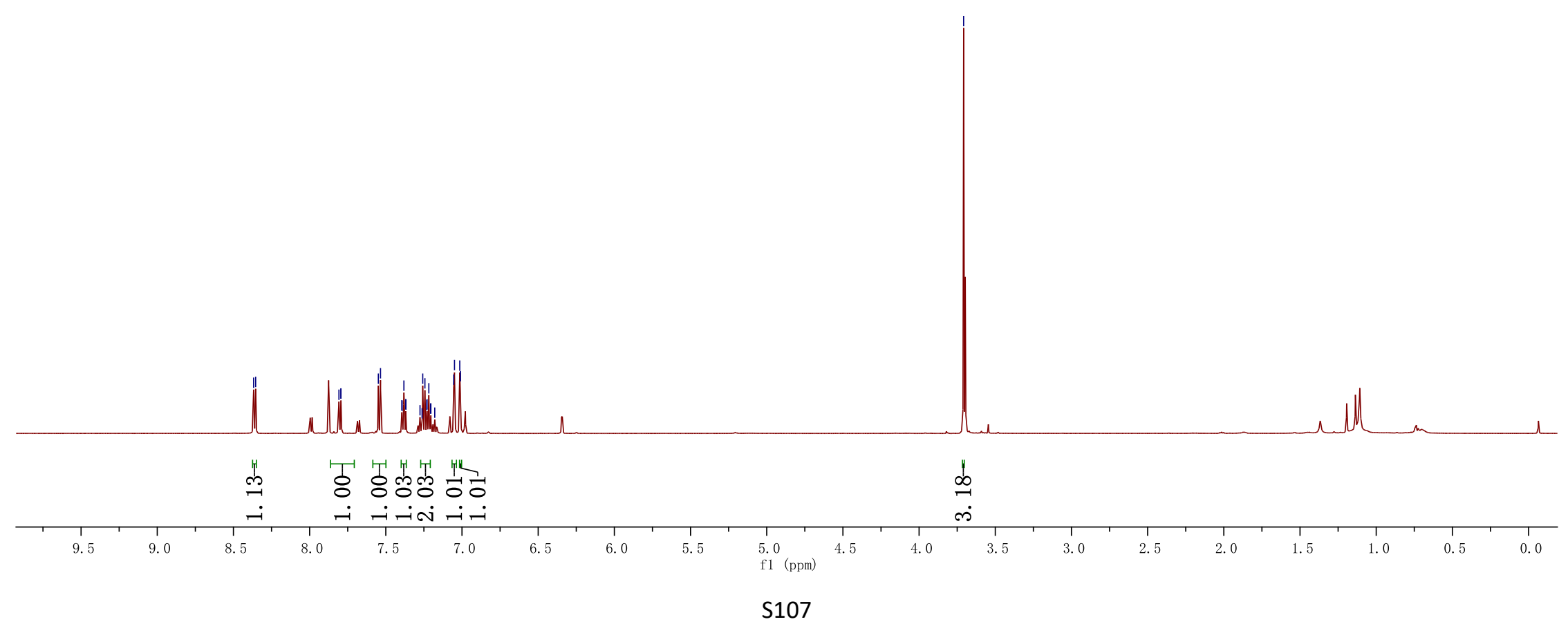




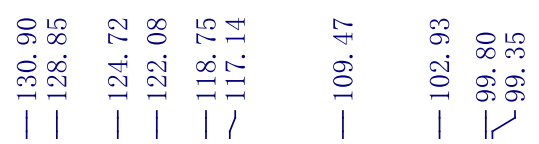

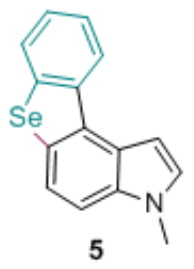

${ }^{13} \mathrm{C}\left\{{ }^{1} \mathrm{H}\right\}$ NMR: $150 \mathrm{MHz}$ solvent: $\mathrm{CDCl}_{3}$

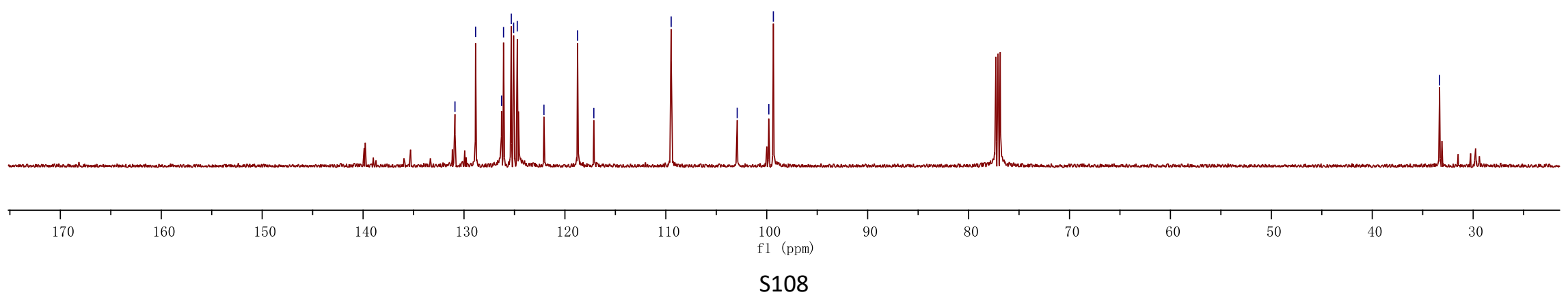




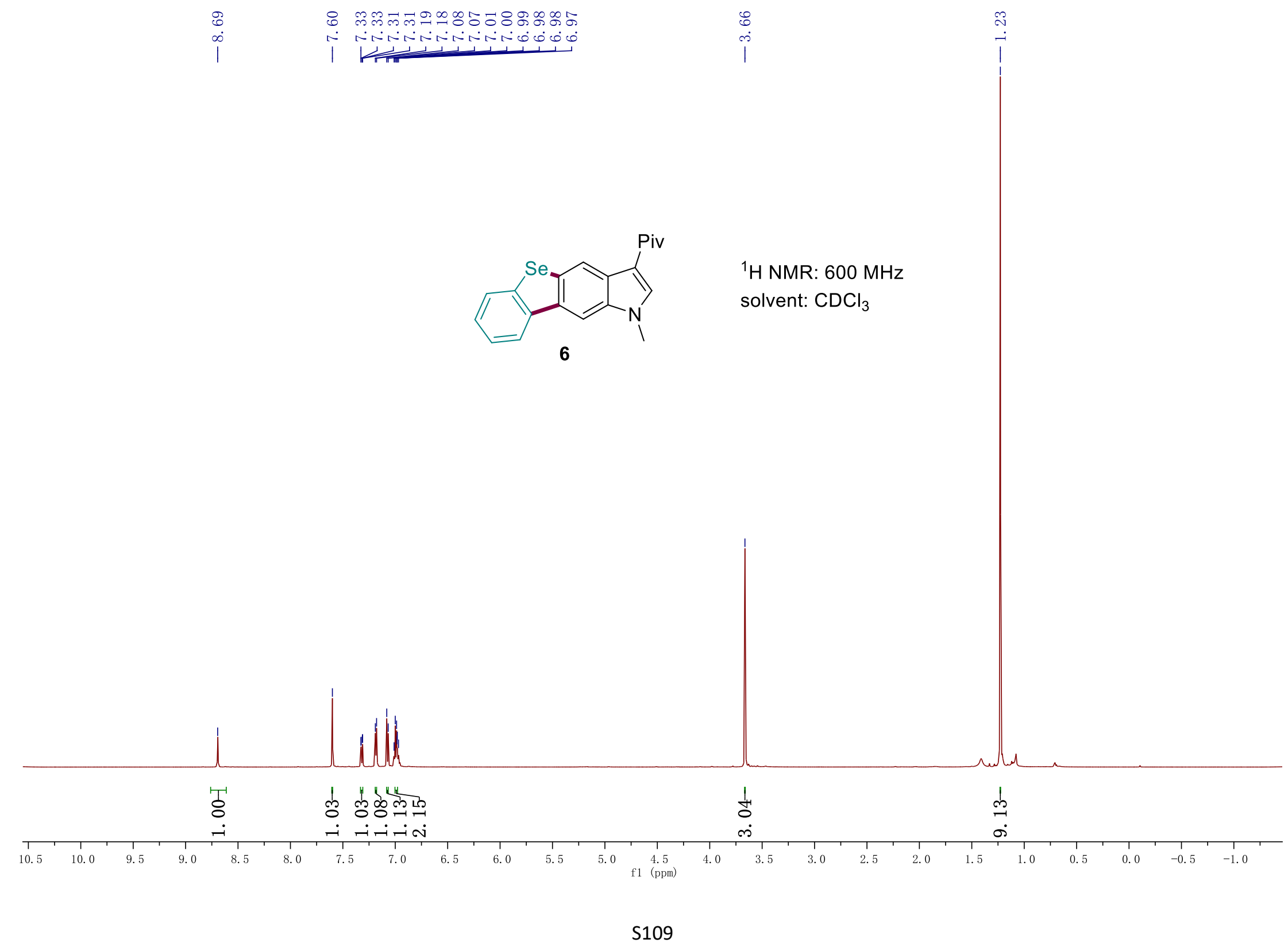




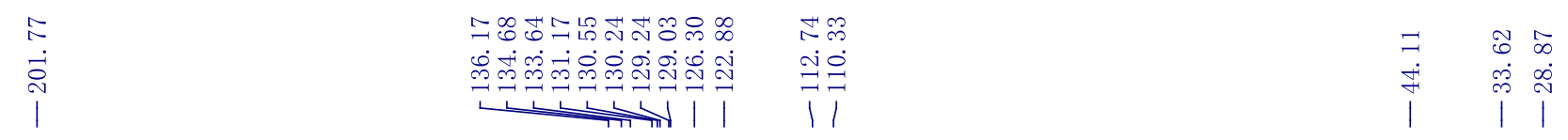

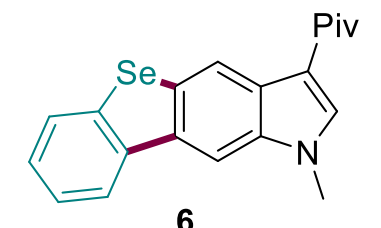

6

${ }^{13} \mathrm{C}\left\{{ }^{1} \mathrm{H}\right\}$ NMR: $150 \mathrm{MHz}$

solvent: $\mathrm{CDCl}_{3}$
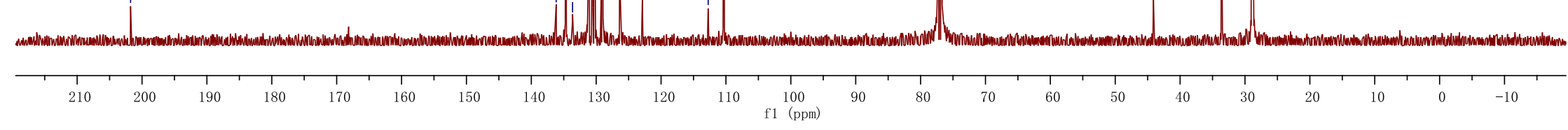